Supporting Information

$O f$

\title{
Building Nanowires from Micelles: Hierarchical Self-assembly of Alternating Amphiphilic Glycopolypeptide Brushes with Pendants of High-Mannose Glycodendron and Oligophenylalanine
}

Yijiang Liu, Yufei Zhang, Zheyu Wang, Jue Wang, Kongchang Wei, Guosong Chen*, Ming Jiang

The State Key Laboratory of Molecular Engineering of Polymers and Department of

Macromolecular Science, Fudan University, Shanghai, 200433 China

Email: guosong@fudan.edu.cn 


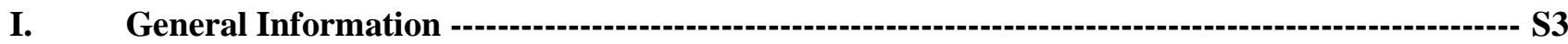

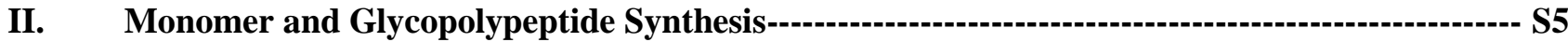

III. Molecular Weight Characterization --

IV. Atomic Force Microscope (AFM) - S12

V. Transmission electron microscope (TEM) images -

VI. Circular dichroism (CD) -

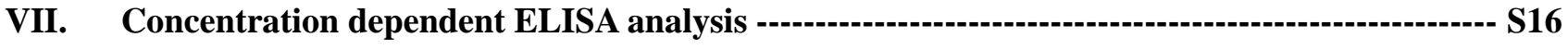

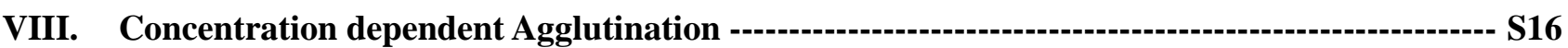

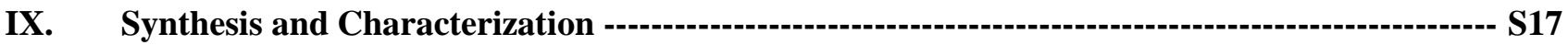

$\mathrm{X} . \quad{ }^{1} \mathrm{H}$ and ${ }^{13} \mathrm{C}$ NMR spectra --- 


\section{General Information}

\section{Materials.}

Chemicals were purchased from J\&K Chemical, TCI and GL Biochem (Shanghai) and used without further purification. 2-O-( $\alpha$-D-Mannopyranosyl)-D-mannopyranose (Mana1-2Man) were purchased from Carbosynth. The extraction of 2-O-( $\alpha$-D-Mannopyranosyl)-2-O-( $\alpha$-D-Mannopyranosyl)-D-mannopyranose

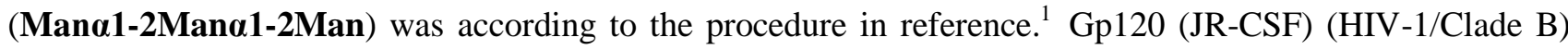
was purchased from Eenzyme (IV-0025-005P). 2G12 recombinant human monoclonal antibody to HIV-1 gp120 was purchased from polymun (AB002). Rabbit anti-Human IgG (H+L) Secondary Antibody, HRP conjugate was purchased from invitrogen (A18903). ELISA Assay Diluent (5X) was purchased from biolegend (421203).

\section{Characterization.}

${ }^{1} \mathbf{H}$ NMR and ${ }^{13} \mathbf{C}$ NMR spectra were taken on a $400 \mathrm{MHz}$ Bruker instrument, and the acquired NMR data were analyzed with Bruker Topspin software. Chemical shift values were referenced using Tetramethylsilane (TMS). Water suppression was used to increase the signal to noise ratio for some polymer samples.

Gel permeation chromatography (GPC) was carried out on a system comprising a Waters 1515 HPLC pump, Waters 2414 refractive index detector, TOSOH TSK gel $\alpha-3000$ and $\alpha-2500$ columns in series at $80^{\circ} \mathrm{C}$ for DMF $(0.2 \% \mathrm{LiBr}$, w/w) phase online test. Waters 1515 HPLC pump, Wyatt DAWN HELEOS II eighteen-angle laser light scattering detector, Wyatt Optical lab refractive index detector, Pw-3000 and $\mathrm{Pw}-2500$ columns in series as $25^{\circ} \mathrm{C}$ for water $\left(0.8 \% \mathrm{NaNO}_{3}\right.$, w/w) phase online test. The offline $\mathrm{dn} / \mathrm{dc}$ tests were performed on Wyatt Optical lab refractive index detector.

Dynamic light scattering (DLS) was taken by Zeta sizer Nano ZS90 from Malvern Instruments.

Matrix Assisted Laser Desorption Ionization-Time of Flight (Maldi-TOF) Mass Spectrum was taken by AB SCIEX 5800 instrument.

Circular dichroism (CD) spectra were taken by a JASCO-815 instrument with a $1 \mathrm{~mm}$ cuvette.

Atomic Force Microscope (AFM) was operated in air on a Bruker Multimode VIII SPM equipped with a J scanner. Experiments were performed in tapping mode with NSC11 tip (spring constant $48 \mathrm{~N} / \mathrm{m}$, MikroMasch). Sample (0.1 mL) was dropped on freshly cleaved mica for AFM test under dry conditions and stayed for drying in air for $24 \mathrm{~h}$.

Transmission electron microscopy (TEM) images were taken with a Tecnai G2 instrument (200 kV) and JEOL TEM-2010 (80kv). Cryo-TEM was performed on JEOL TEM-2100F (200 kV). Field Emission Transmission Electron Microscope (FETEM) was taken with Tecnai G2 F20 S-Twin (200kv).

The cryofixation TEM sample prepared as followed procedure: the hydrophilic copper grid was put on a cupper cubic that half-immersed in liquid nitrogen. $2 \mu \mathrm{L}$ of $0.2 \mathrm{mg} / \mathrm{mL}$ sample solution was dropped on the cupper grid. The sample solution was immediately frozen and put into lyophilizer to give the dry cryofixation TEM sample.

Enzyme Linked Immunosorbent Assay (ELISA) The procedure was following reference ${ }^{2}$. Briefly, microtiter plate wells (flat bottom, Costar type 3690; Corning) were coated with $25 \mathrm{ng}$ per well gp120 JR-CSF overnight at $4{ }^{\circ} \mathrm{C}$. All subsequent steps were performed at room temperature. The wells then were washed four 
times with $0.05 \%$ (vol/vol) Tween 20 in PBS and blocking for $2 \mathrm{~h}$ with 3\% (mass/vol) BSA in PBS. IgG 2G12, diluted to $0.25 \mu \mathrm{g} / \mathrm{mL}$ (25 ng per well) with $1 \%$ (mass/vol) BSA in PBS, then was added to the antigen-coated wells in the presence of serially diluted glycopolypeptide and AAGBs assemblies starting at a concentration of $8 \mathrm{mM}$ for $2 \mathrm{~h}$. Unbound $\mathrm{Ab}$ was removed by washing four times as described above. Bound $2 \mathrm{G} 12$ was detected with an alkaline phosphatase conjugated goat anti-human IgG F(ab') $)_{2} \mathrm{Ab}$ (Pierce) diluted 1:2000 in PBS-BT. After $1 \mathrm{~h}$, the wells were washed four times, and bound Ab was visualized with p-nitrophenol phosphate substrate and monitored at $405 \mathrm{~nm}$. Concentration dependent ELISA results were listed in Figure S17.

Turbidity test Agglutination of glycopolypeptide and AAGBs assemblies with lectins were monitored in 0.50 $\mathrm{mL}$ semi-micro disposable cuvettes at $23{ }^{\circ} \mathrm{C}$ at single wavelength $\lambda=450 \mathrm{~nm}$ by using a Shimadzu UV-2550 spectrophotometer. Plant lectin ConA (specific for D-mannose) was used as model lectin for agglutination in $10 \mathrm{mM}$ HEPES buffer $\left(\mathrm{pH}=7.4,1.0 \mathrm{mM} \mathrm{CaCl}_{2}\right.$ and $1.0 \mathrm{mM} \mathrm{MnCl}_{2}$ ). The solution of glycopolypeptide or AAGBs assemblies $(20 \mu \mathrm{L})$ was injected into $400 \mu \mathrm{L}$ solution of ConA. The mixture was kept for $2 \mathrm{~s}$ before recording the absorbance change. Concentration dependent UV agglutination results were listed in Figure S18. 


\section{Monomer and Glycopolypeptide Synthesis}
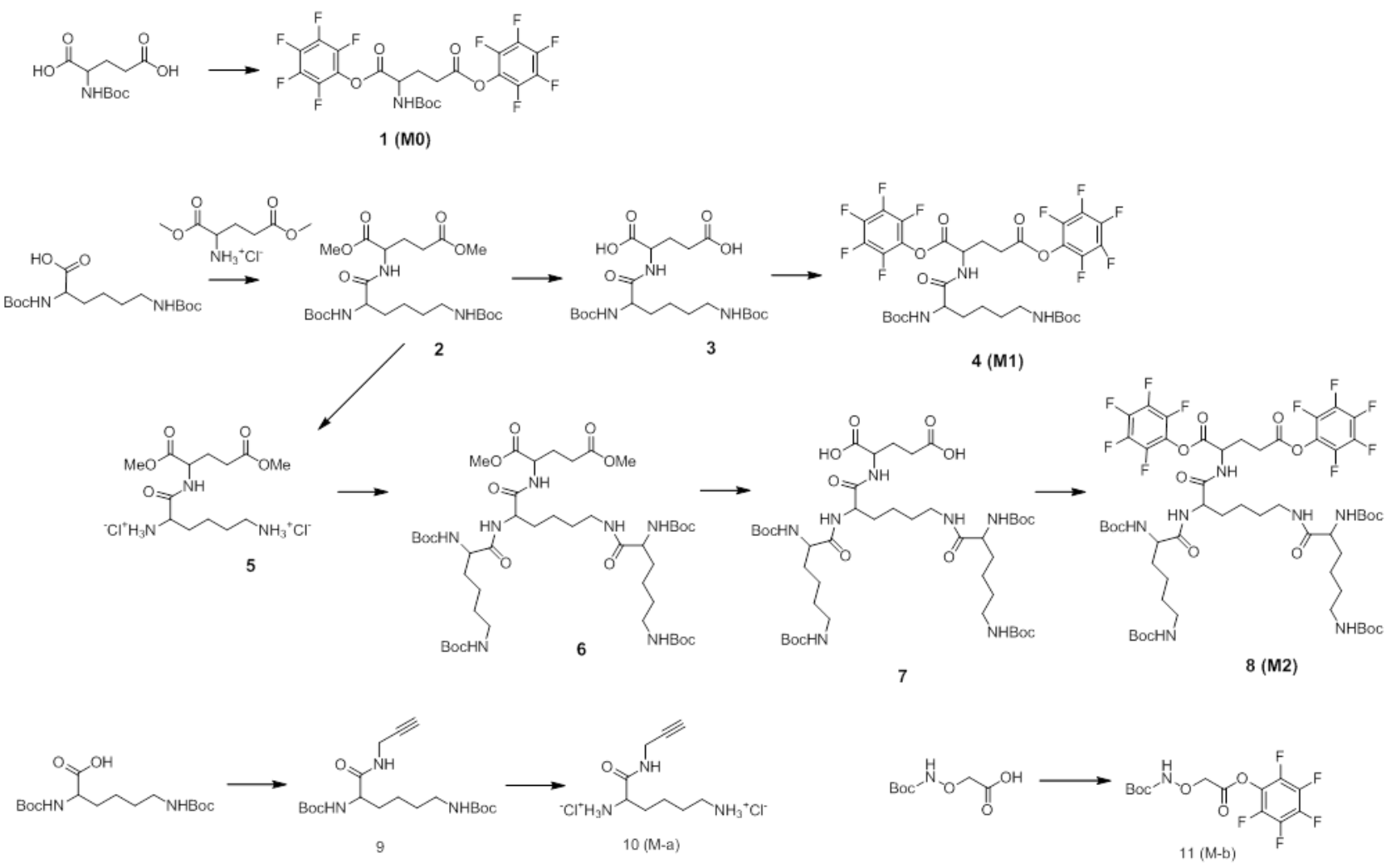

Scheme S1. Synthesis of lysine based dendronized peptide monomer M0-2, M-a and M-b.

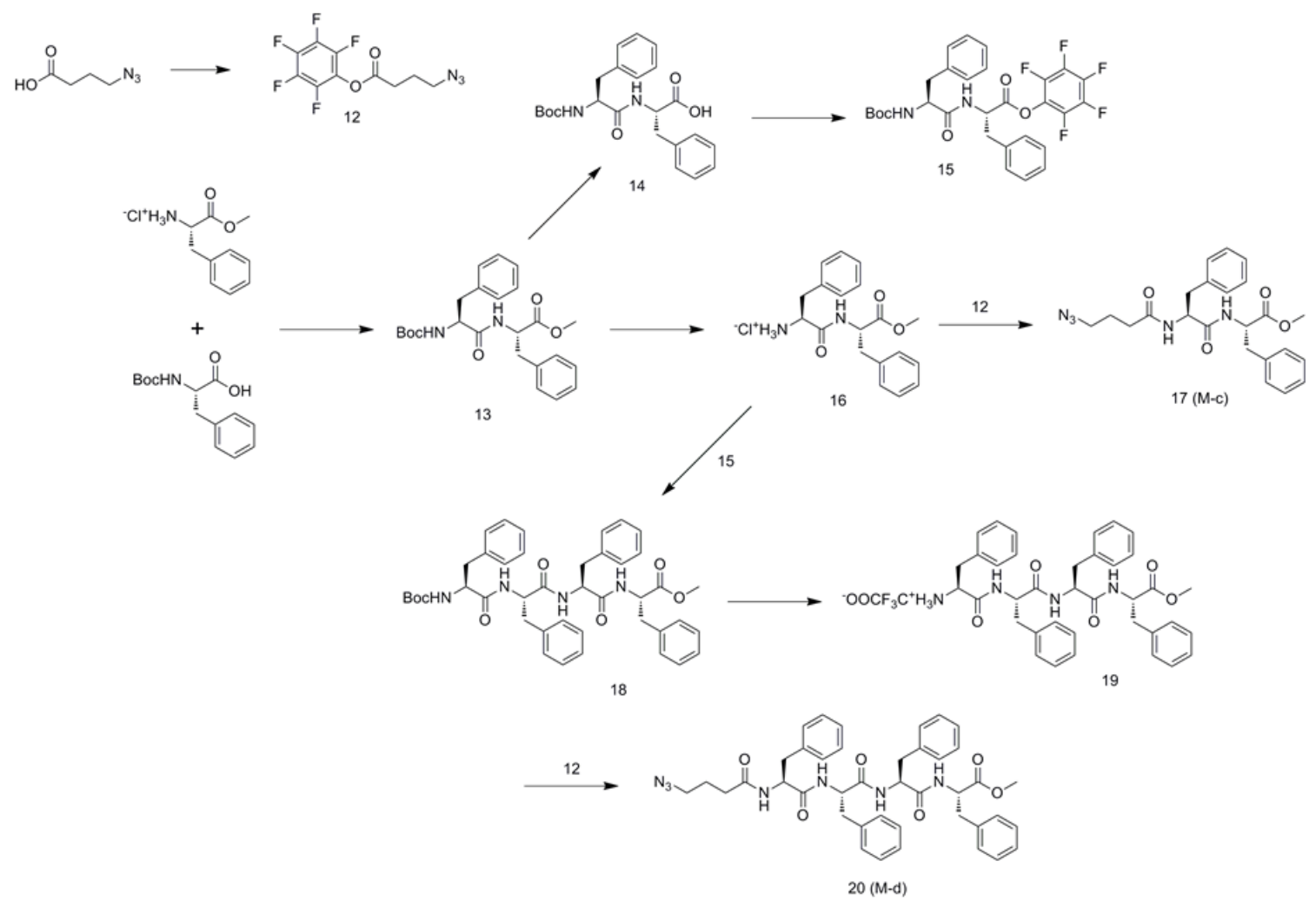

Scheme S2. Synthesis of Azide Functionalized Phenylalanine Tetrapeptide 


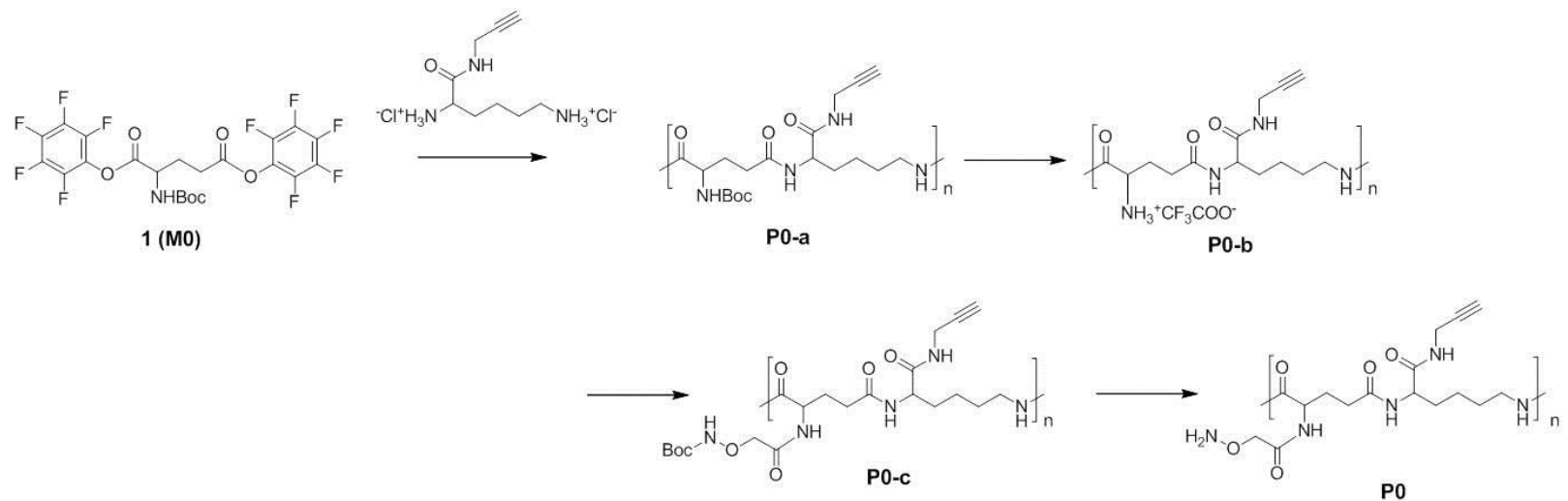

Po
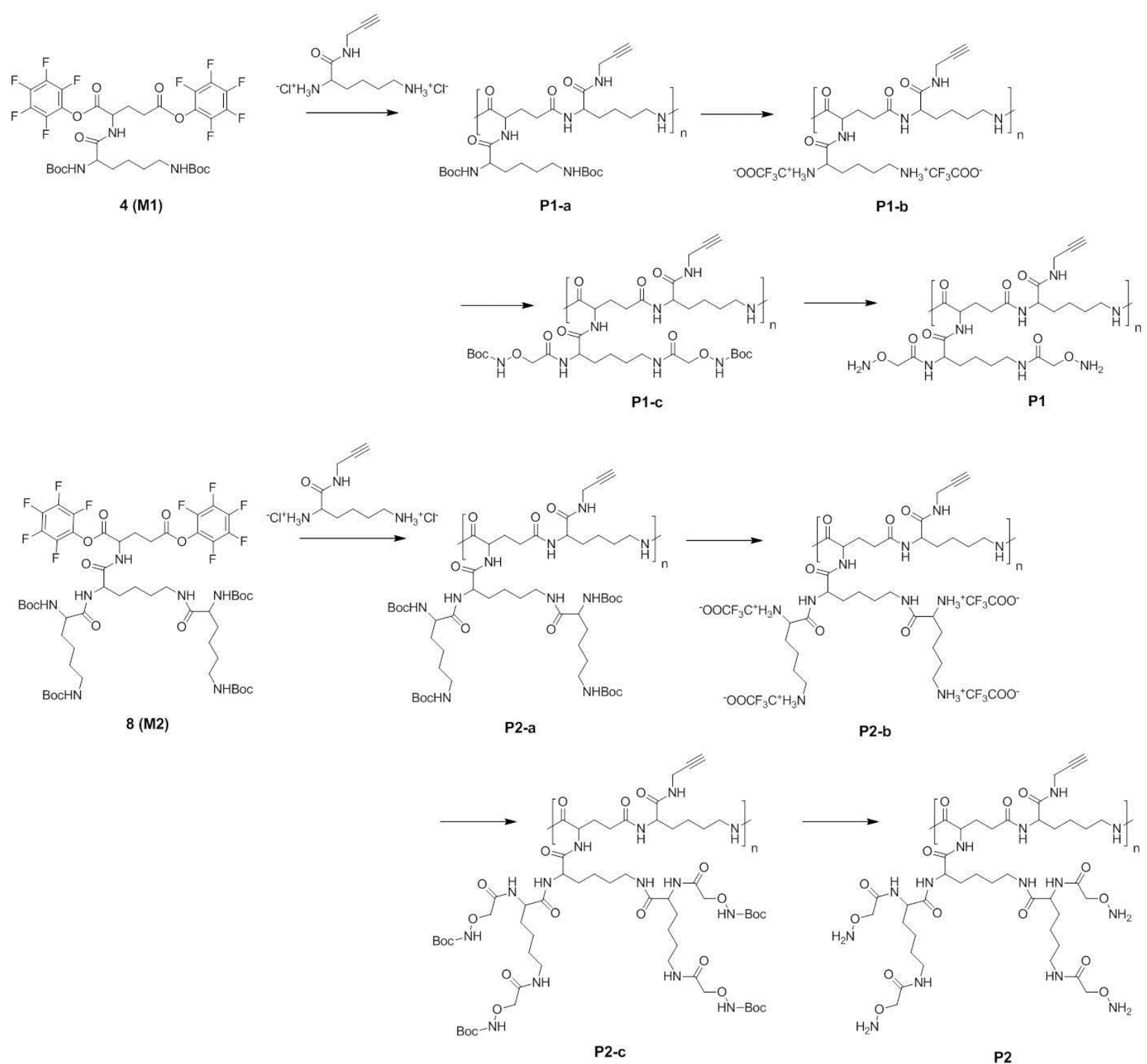

Scheme S3. General Procedure for polymerization and polypeptide post-modification 

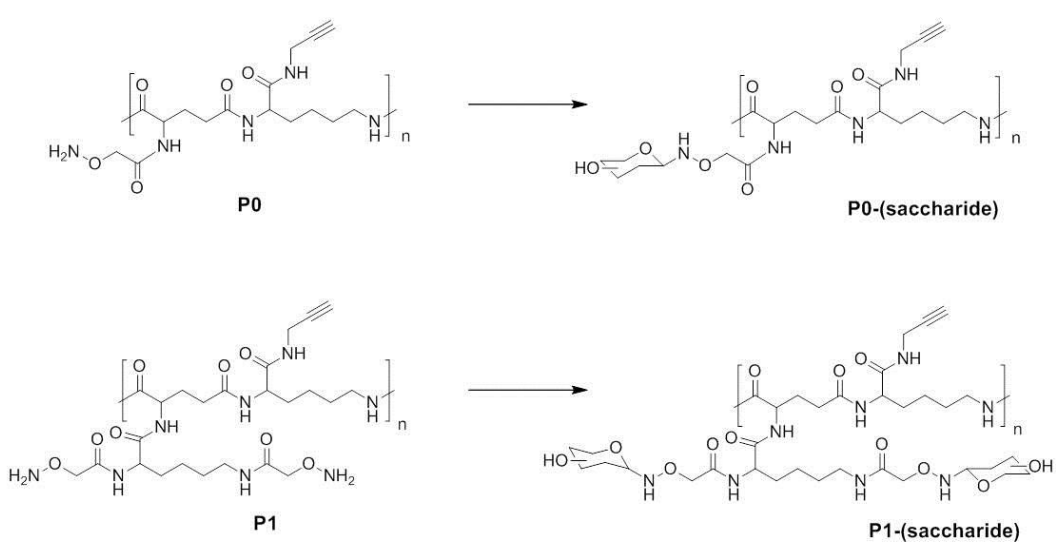

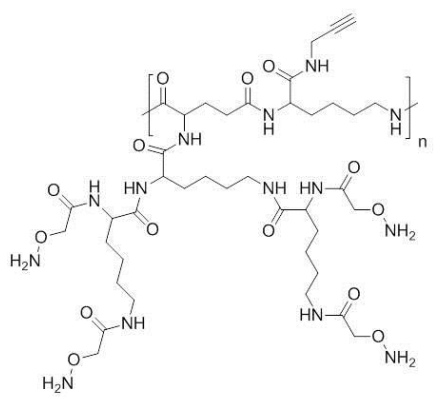

P2

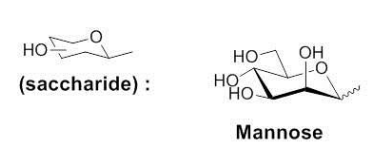

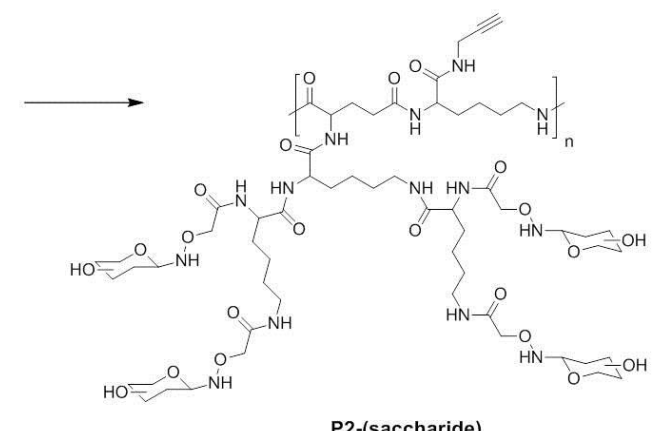

P2-(saccharide)
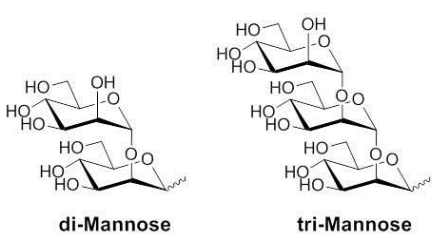

Scheme S4. General Procedure for Glycosylation
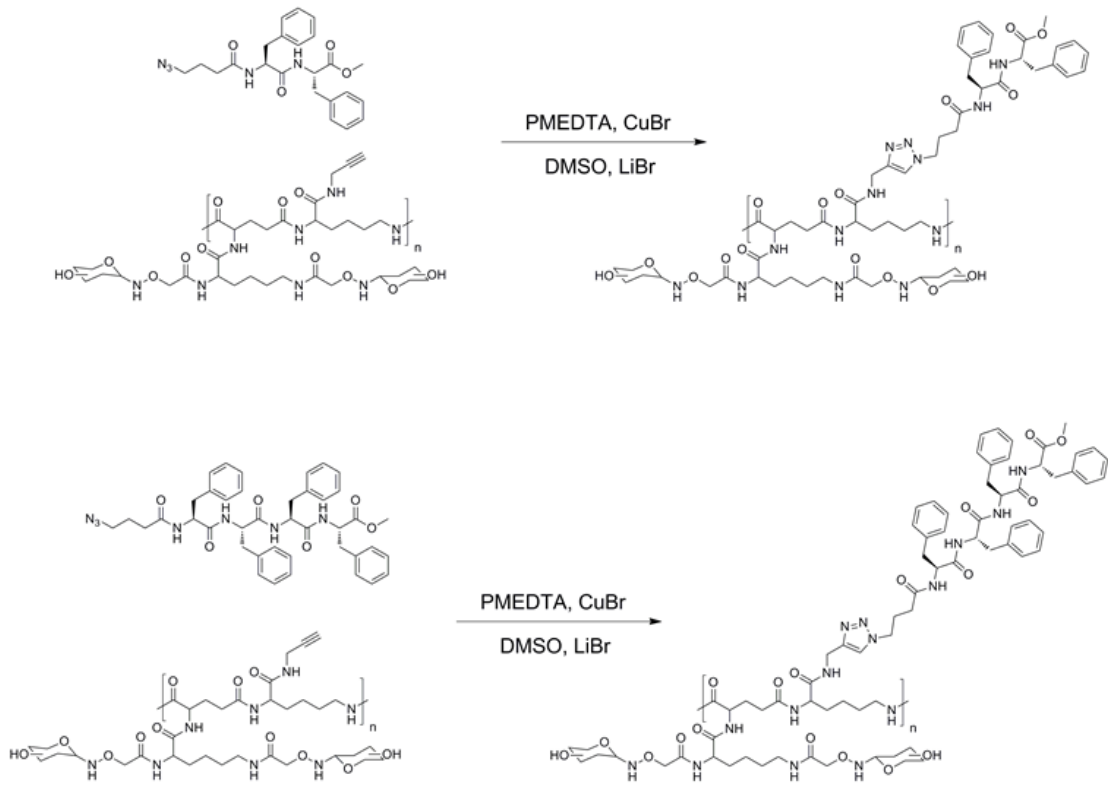

Scheme S5. General Procedure for Amphiphilic Glyco-Dendronized (AGDPs) Polypeptide Synthesis and Self-assembly 


\section{Molecular Weight Characterization}

Table S1. dn/dc and molecular weight given by Refractive Index (RI) and Static Light Scattering (SLS) detectors.

$\begin{array}{cccccc} & \mathbf{d n} / \mathbf{d c} & \mathbf{M n} & \mathbf{M w} & \text { PDI } & \text { DP } \\ \text { P0 } & 0.0928 \pm 0.0012 & 1.390 \times 10^{4} & 1.886 \times 10^{4} & 1.357 & 51 \\ \text { P1 } & 0.0909 \pm 0.0021 & 1.136 \times 10^{4} & 1.655 \times 10^{4} & 1.457 & 29 \\ \text { P2 } & 0.0814 \pm 0.0012 & * & * & * & * \\ \text { P0M } & 0.1653 \pm 0.0010 & 1.108 \times 10^{4} & 2.200 \times 10^{4} & 1.985 & 43 \\ \text { P0dM } & 0.1626 \pm 0.0012 & 1.699 \times 10^{4} & 2.396 \times 10^{4} & 1.41 & 36 \\ \text { P0tM } & 0.1563 \pm 0.0008 & 1.986 \times 10^{4} & 2.641 \times 10^{4} & 1.33 & 32 \\ \text { P1M } & 0.1871 \pm 0.0041 & 1.646 \times 10^{4} & 2.669 \times 10^{4} & 1.622 & 30 \\ \text { P1dM } & 0.1644 \pm 0.0008 & 1.833 \times 10^{4} & 2.646 \times 10^{4} & 1.444 & 22 \\ \text { P1tM } & 0.1483 \pm 0.0005 & 2.024 \times 10^{4} & 3.184 \times 10^{4} & 1.573 & 21 \\ \text { P2M } & 0.1724 \pm 0.0029 & 2.206 \times 10^{4} & 4.843 \times 10^{4} & 2.196 & 30 \\ \text { P2dM } & 0.1675 \pm 0.0004 & 2.570 \times 10^{4} & 5.277 \times 10^{4} & 2.053 & 25 \\ \text { P2tM } & 0.1616 \pm 0.0007 & 3.061 \times 10^{4} & 5.692 \times 10^{4} & 1.859 & 21\end{array}$

* The molecular weight of $\mathbf{P 2}$ can hardly be measured due to its poor solubility in DMF.

Table S2. Covalent Coupling of Reducing Oligo-Mannose and Corresponding Polypeptide.

\begin{tabular}{lcccccc} 
Sample & & & & \multicolumn{3}{c}{ Isomeric ratio $(\%)^{\mathrm{b}}$} \\
Name & $\mathrm{M}_{\mathrm{w}}(\mathrm{kDa})$ & $\mathrm{DP}$ & 1. e. $(\%)^{\mathrm{a}}$ & $\alpha / \beta$ & $($ E)-oxime & $(\mathrm{Z})$-oxime \\
P0M & 22.0 & 43 & 87.1 & 14.5 & 70.2 & 15.3 \\
P1M & 24.0 & 36 & 90.0 & 13.3 & 71.7 & 15.0 \\
P2M & 26.4 & 32 & 95.8 & 16.2 & 68.2 & 15.6 \\
P0dM & 26.7 & 30 & 100.0 & 14.1 & 71.0 & 15.0 \\
P1dM & 26.5 & 22 & 96.5 & 13.8 & 71.1 & 15.1 \\
P2dM & 31.8 & 21 & 94.0 & 14.3 & 71.9 & 13.8 \\
P0tM & 48.4 & 30 & $-\mathrm{c}$ & $-\mathrm{c}$ & 79.2 & 20.8 \\
P1tM & 52.8 & 25 & - & - & 83.2 & 16.8 \\
P2tM & 56.9 & 21 & - & - & 81.2 & 18.8
\end{tabular}

${ }^{\mathrm{a}}$ 1.e. $=$ ligation efficiency for the reaction of peptide scaffold with 1.2 equiv. of oligomannose, ${ }^{\mathrm{b}}$ Isomeric ratio determined by ${ }^{1} \mathrm{H}$ NMR analysis. ${ }^{c}$ For trimannoside-modified polypeptides, mannosylamine content cannot be determined due to signal overlapping. The specific $\alpha / \beta$ conformation could not be determined from ${ }^{1} \mathrm{H}$ NMR. 

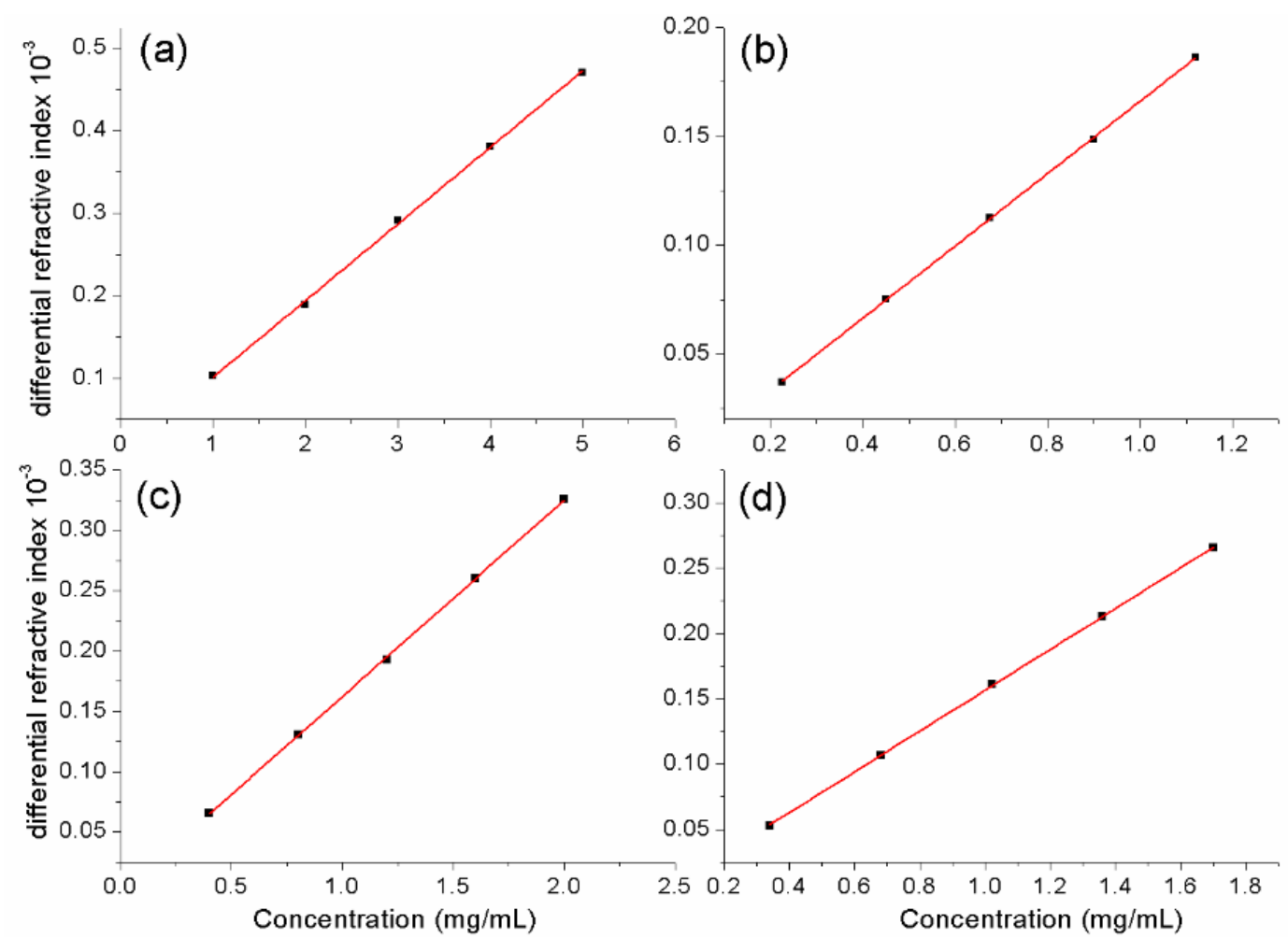

Figure S1. The dn/dc results of (a) P0, (b) P0M, (c) POdM and (d) P0tM in DMF/water.
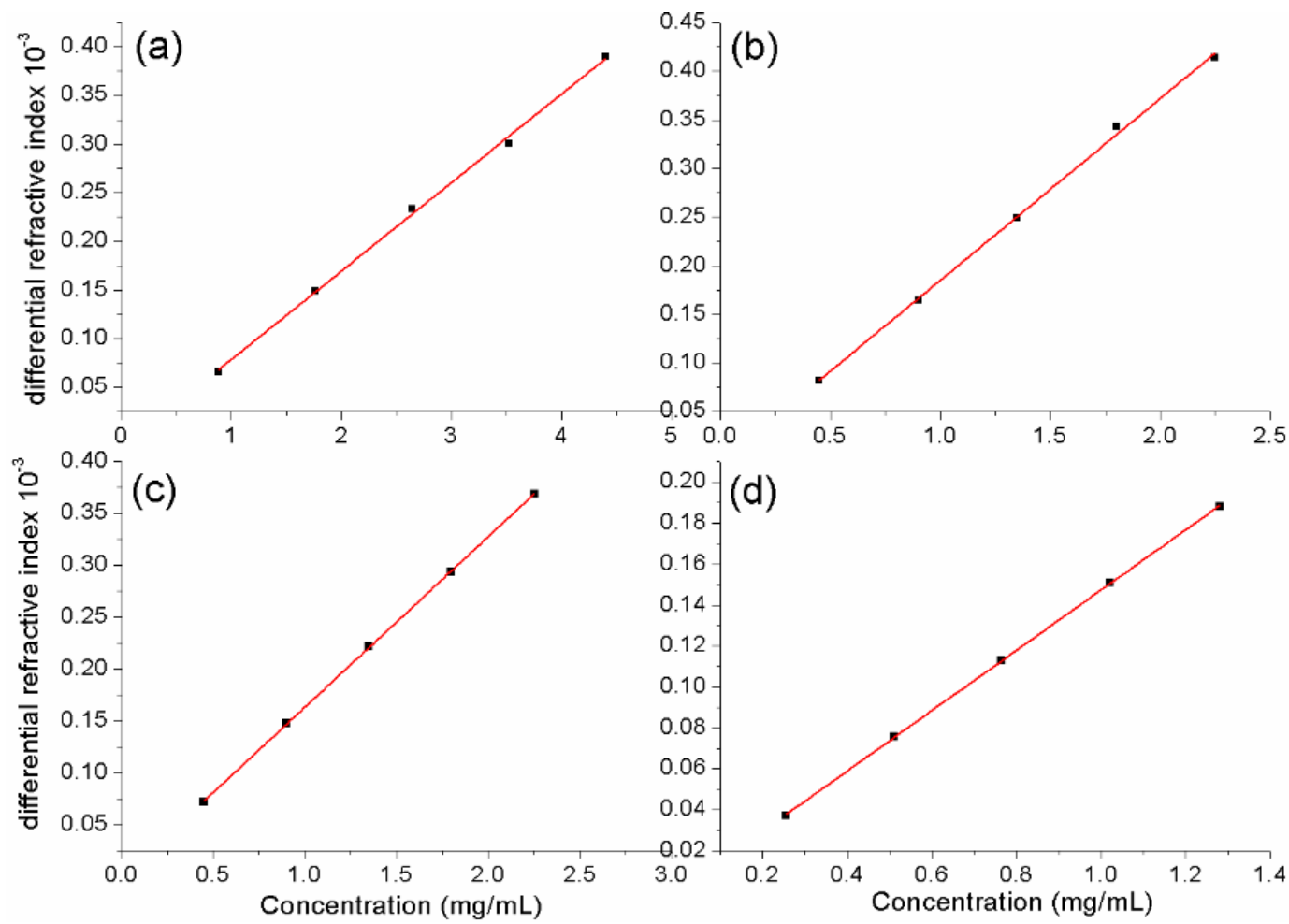

Figure S2. The dn/dc results of (a) P01, (b) P1M, (c) P1dM and (d) P1tM in DMF/water. 

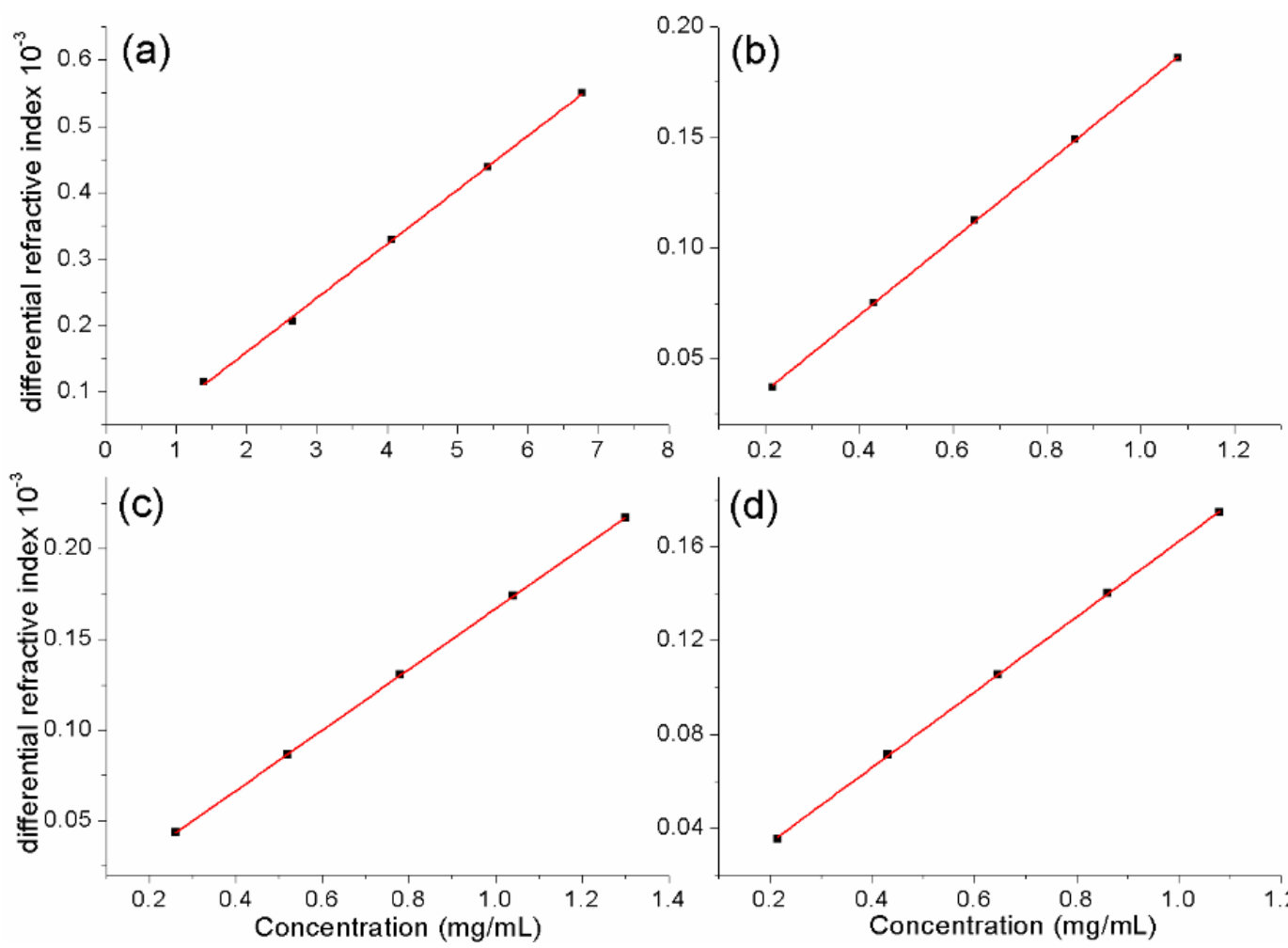

(d)

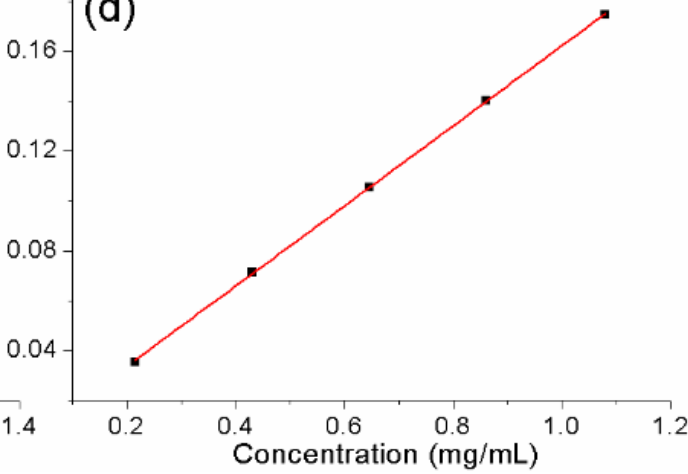

Figure S3. The dn/dc results of (a) P2, (b) P2M, (c) P2dM and (d) P2tM in DMF/water.
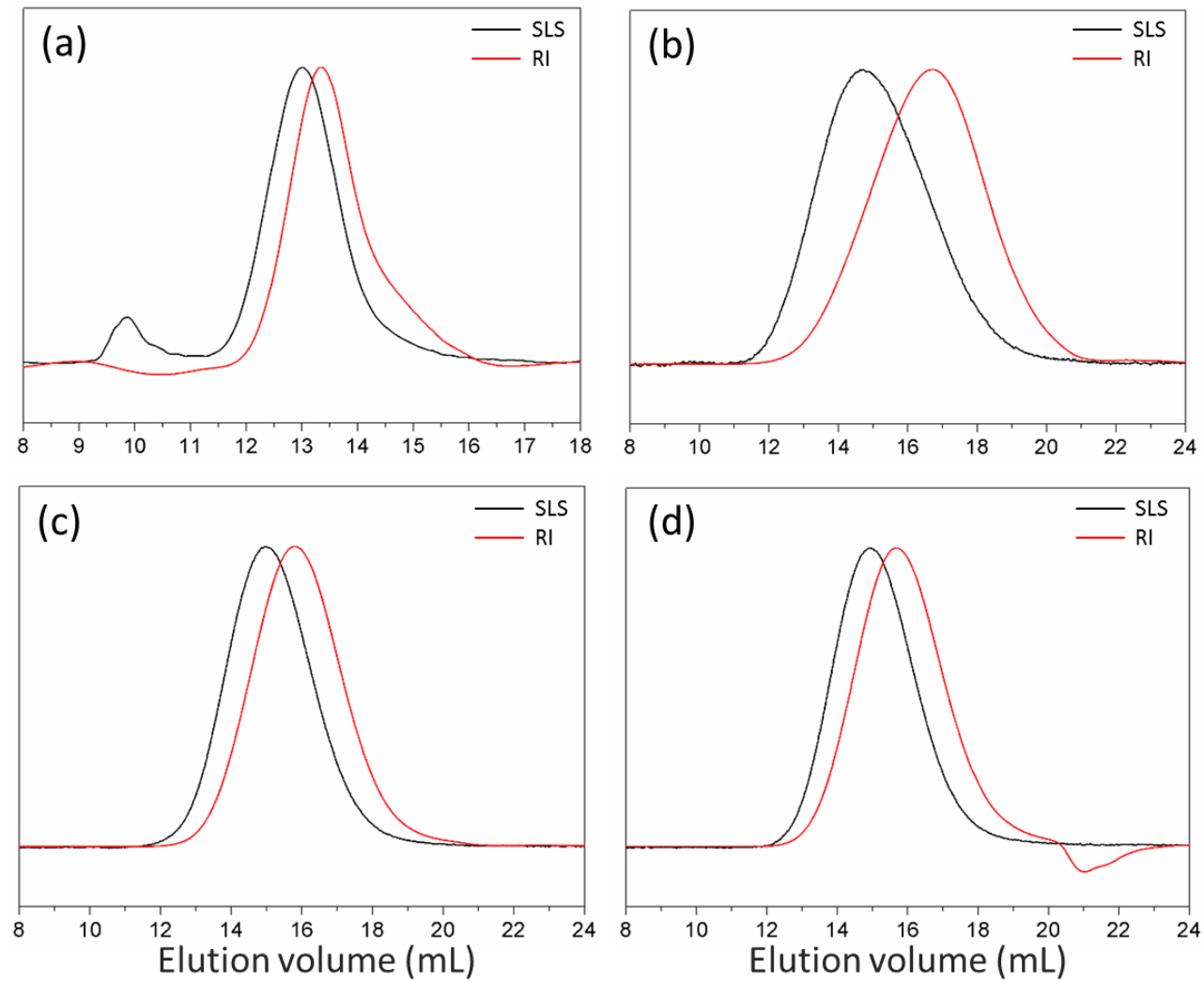

Figure S4. GPC traces of (a) P0, (b) P0M, (c) P0dM and (d) P0tM monitored by Refractive Index (RI) and Static Light Scattering (SLS) detectors in DMF. 

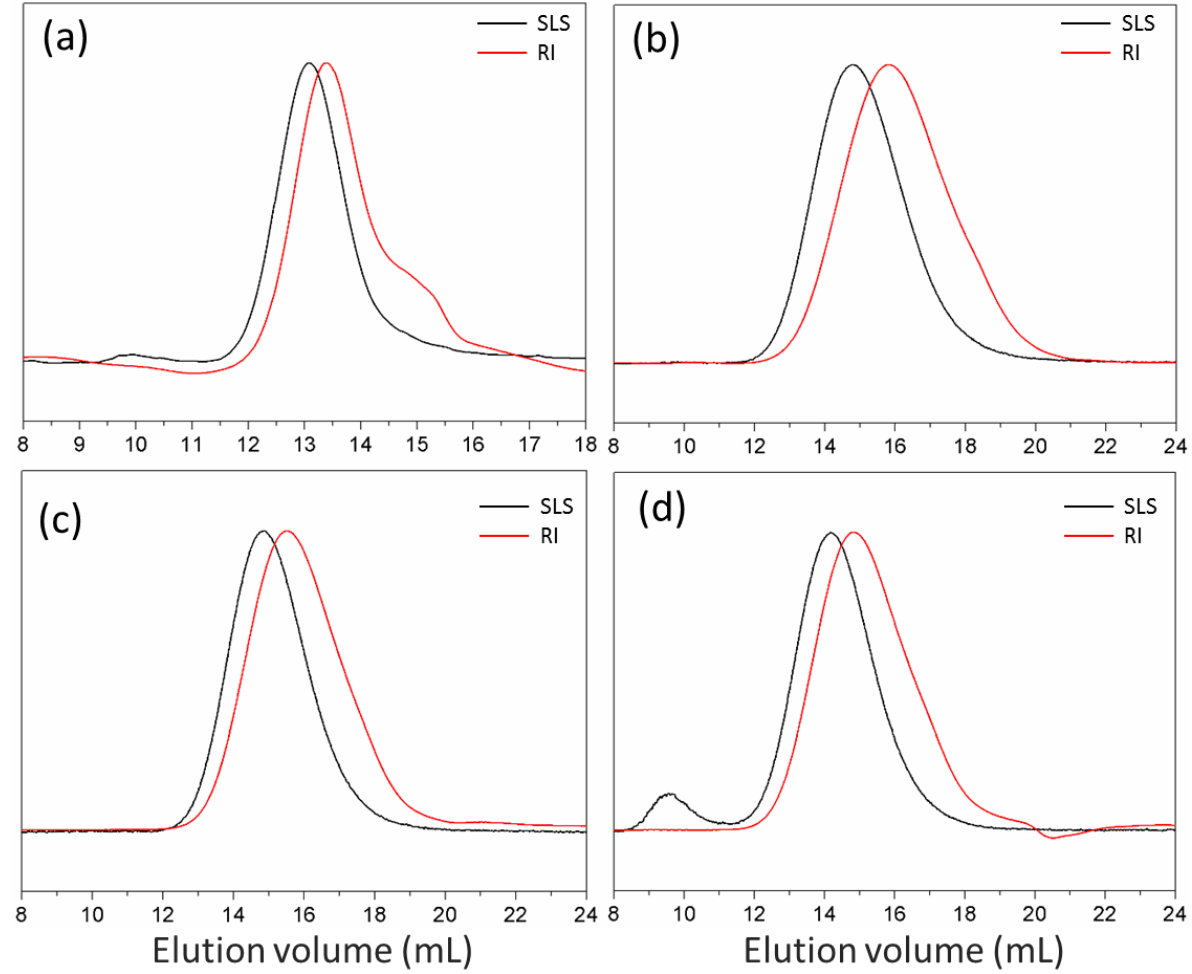

Figure S5. GPC traces of (a) P1, (b) P1M, (c) P1dM and (d) P1tM monitored by Refractive Index (RI) and Static Light Scattering (SLS) in water.
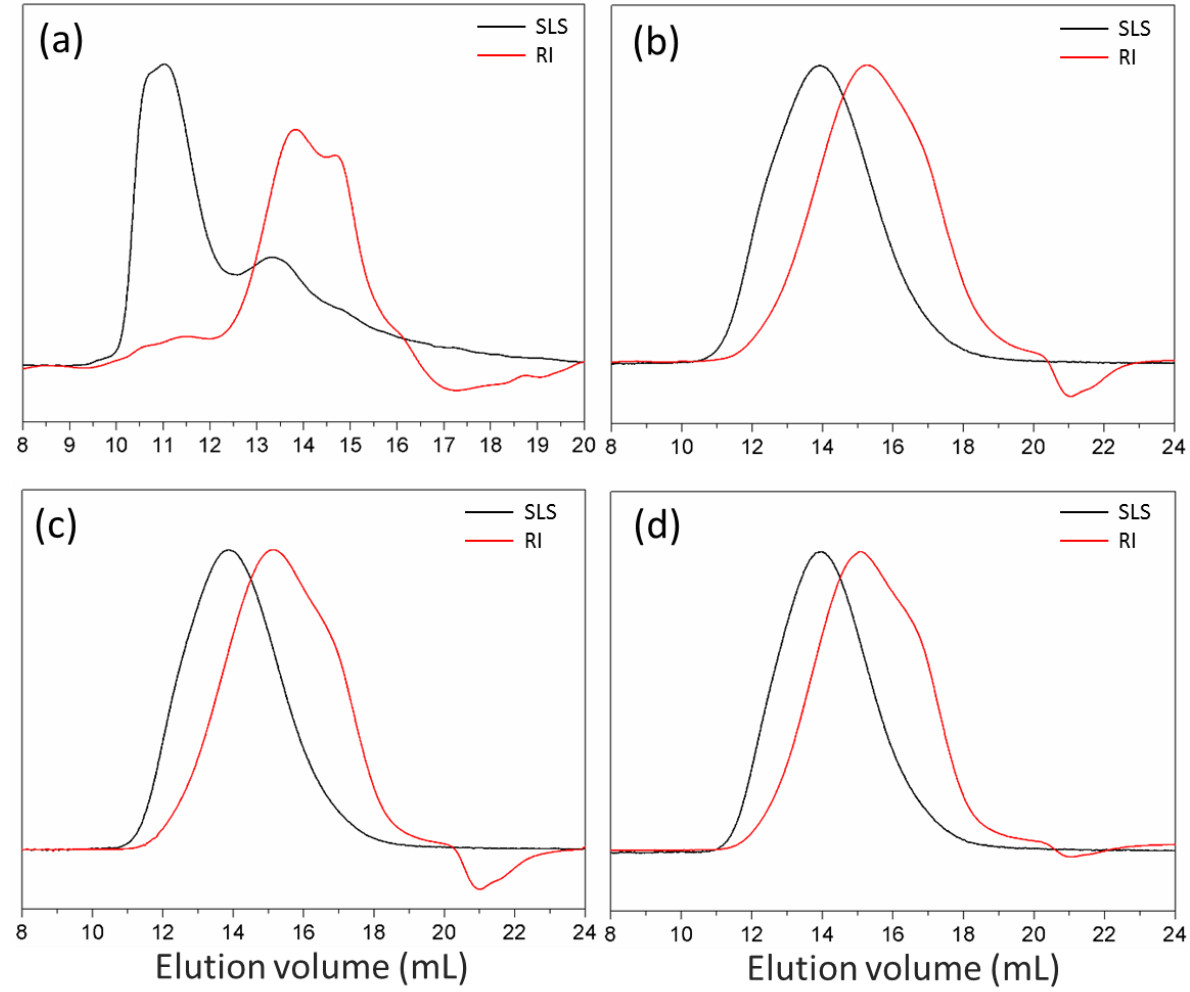

Figure S6. GPC traces of (a) P2, (b) P2M, (c) P2dM and (d) P2tM monitored by Refractive Index (RI) and Static Light Scattering (SLS) in water phase 
IV. Atomic Force Microscope (AFM)
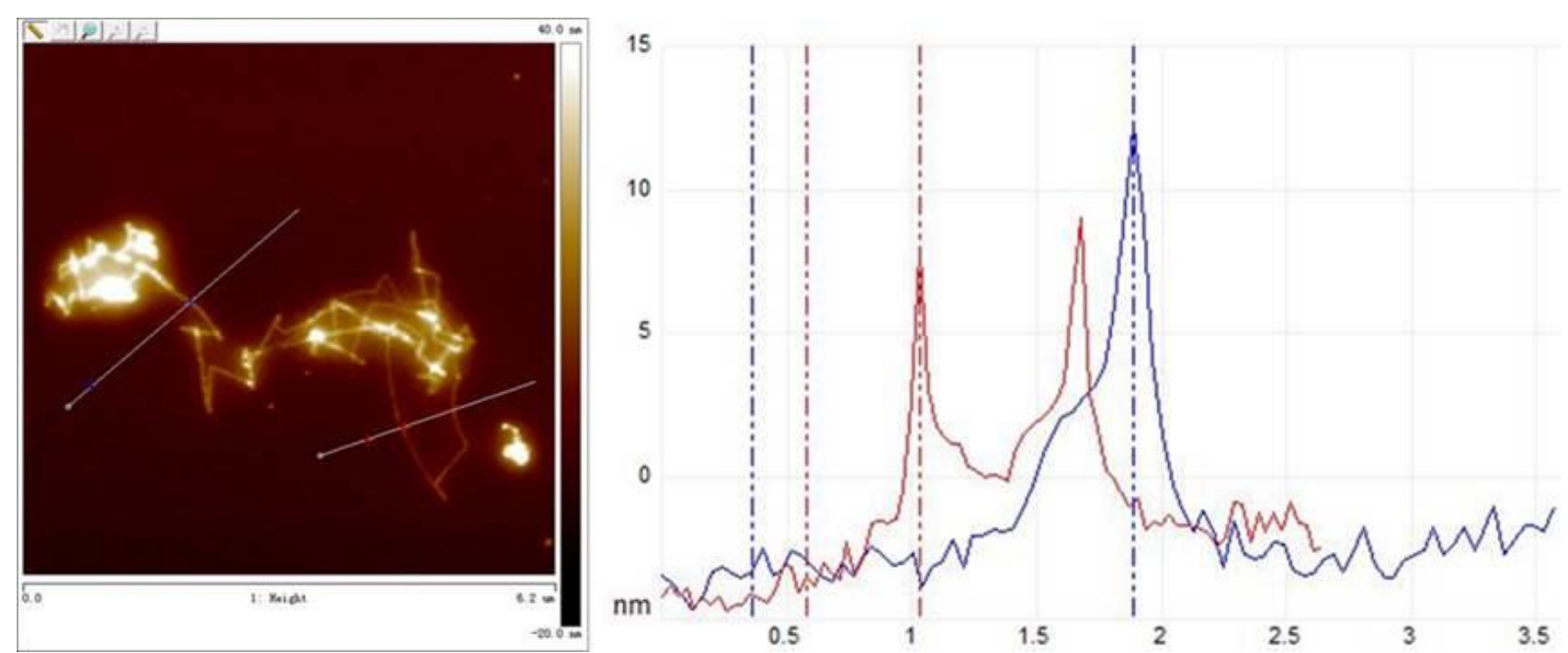

Figure S7. AFM images of P1tM-F 4 .

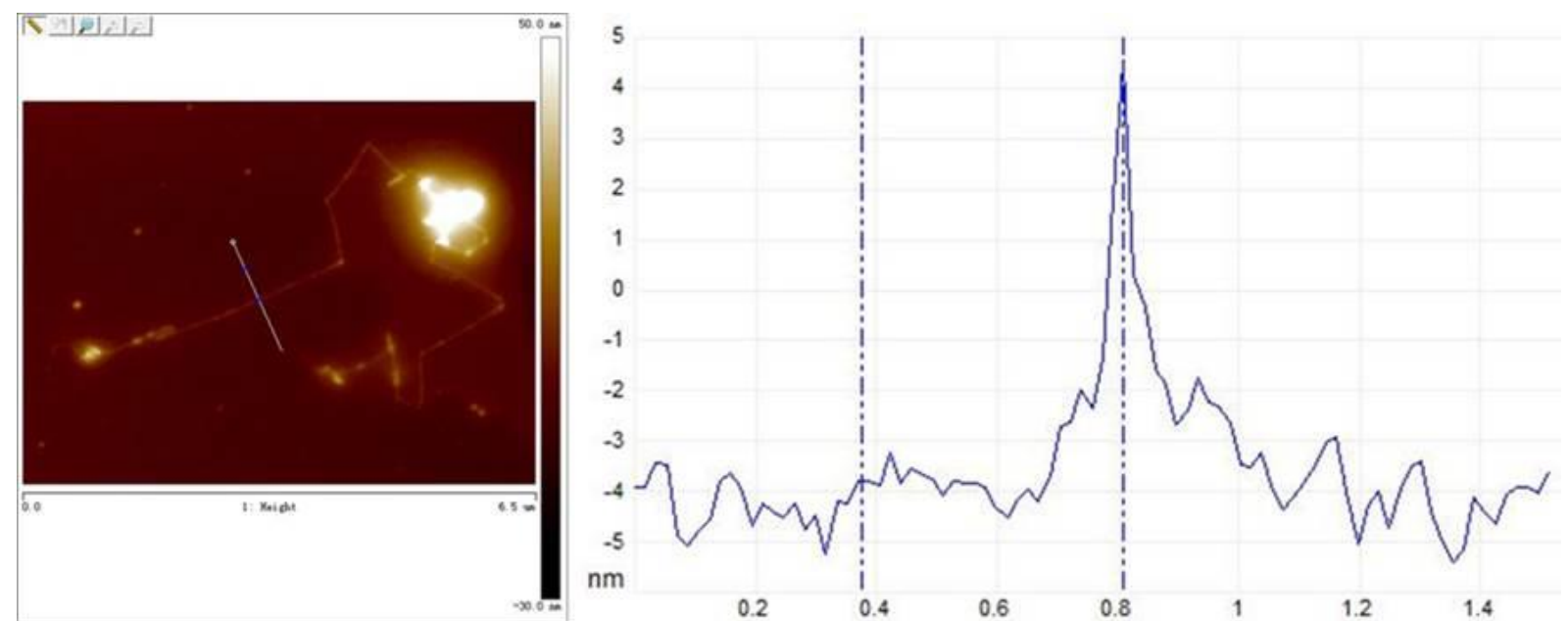

Figure S8. AFM images of P2tM-F 4 . 
V. Transmission electron microscope (TEM) images and Dynamic Light Scattering (DLS)
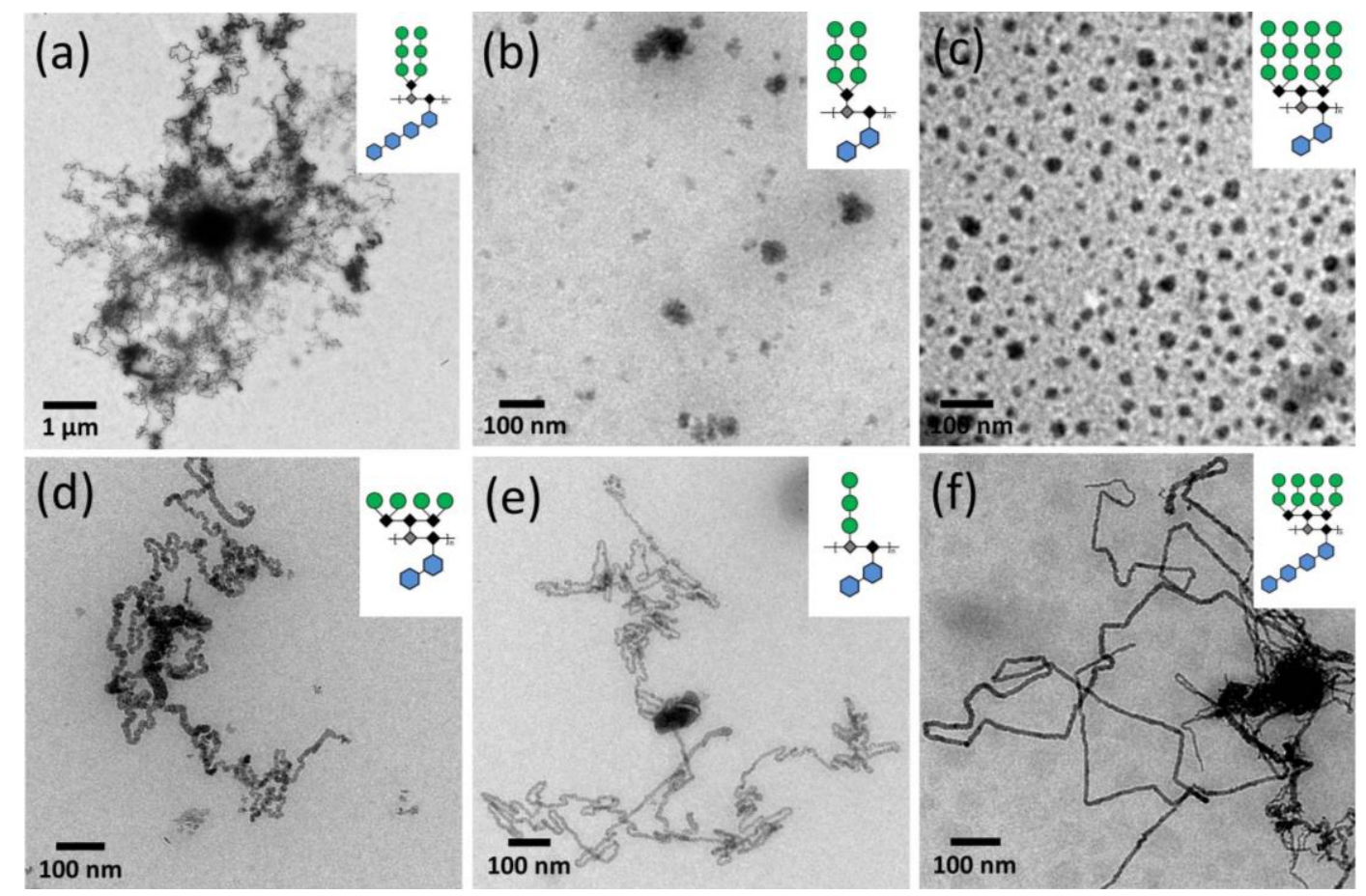

Figure S9. TEM images of AAGBs assemblies.

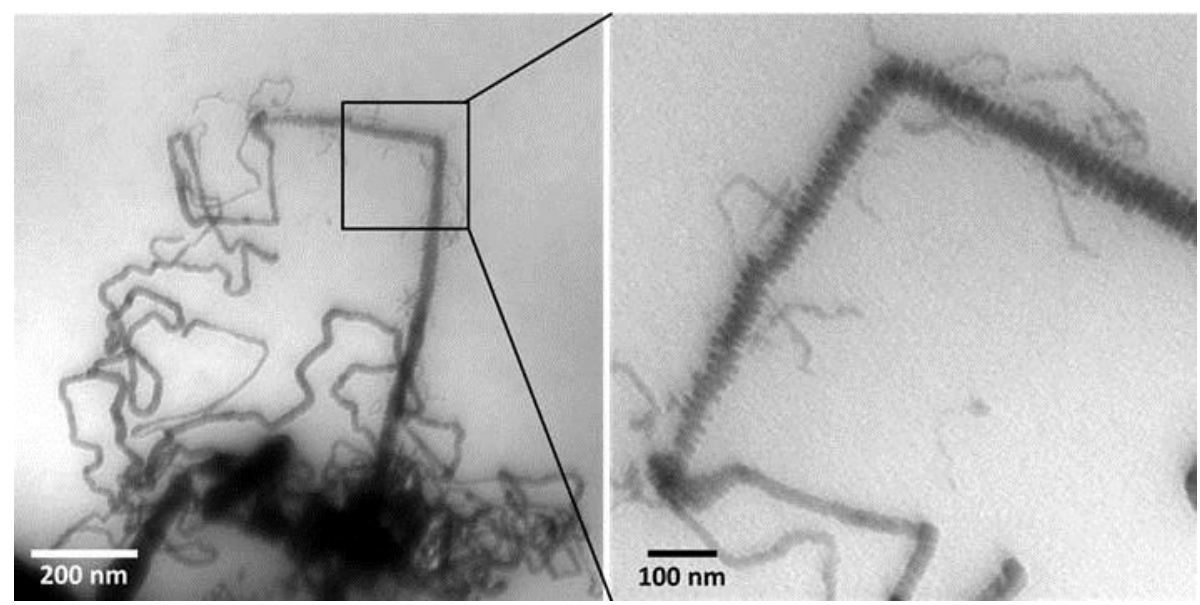

Figure S10. TEM images of P2tM-F 4 intermediate state (80kV TEM). 


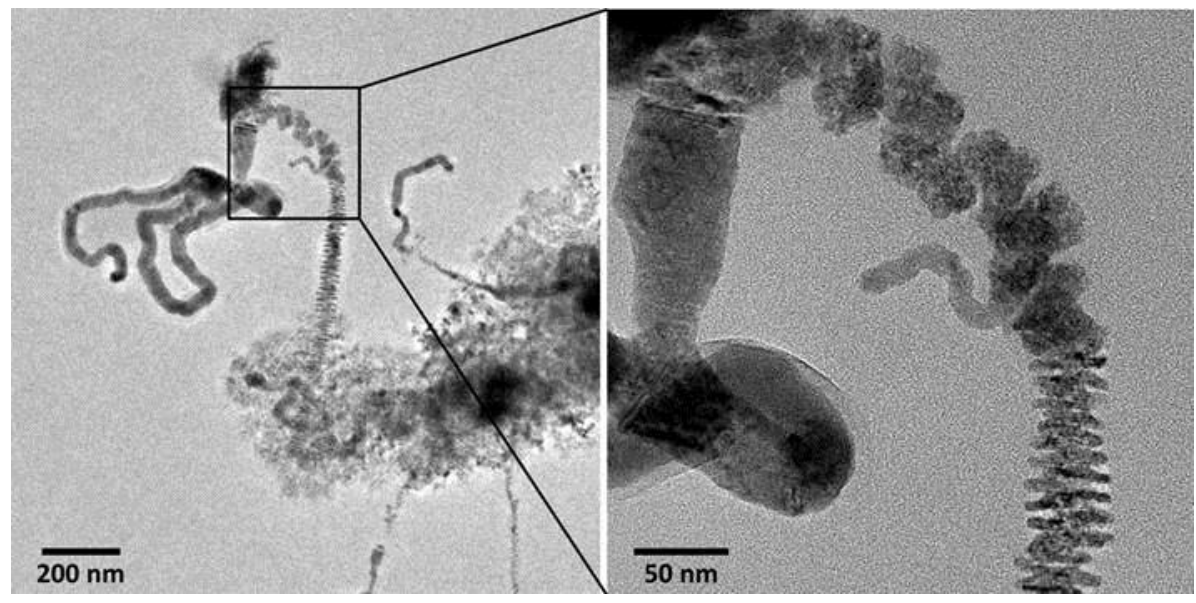

Figure S11. Field Emission Transmission Electron Microscope (FETEM, 200kV) images of P2tM-F 4 intermediate state.

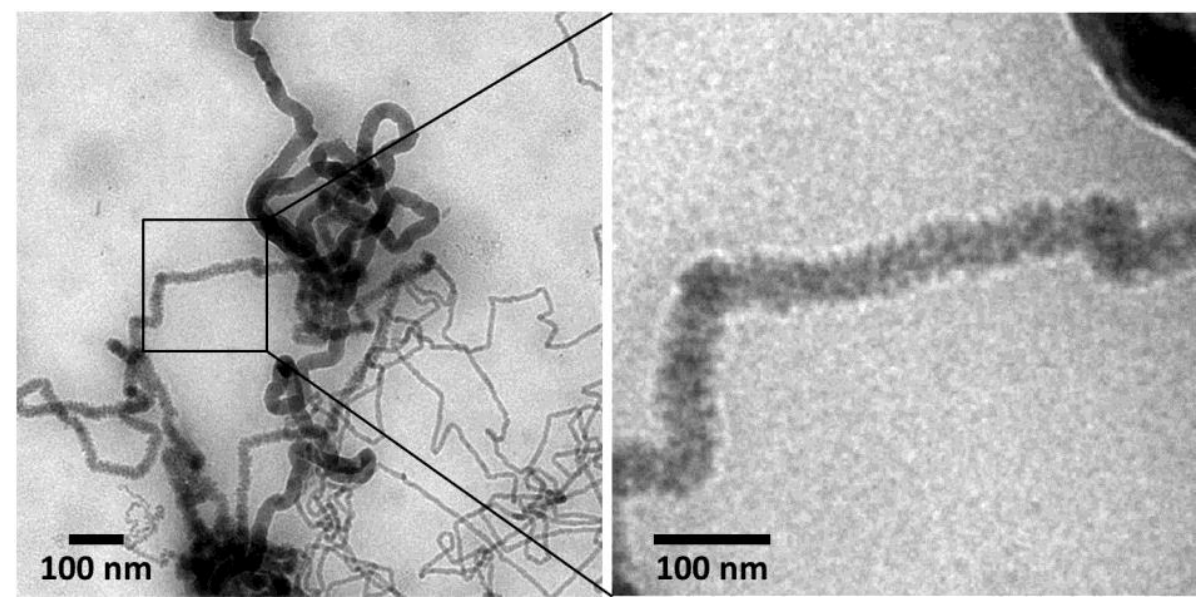

Figure S12. TEM images of P1tM-F 4 intermediate state.

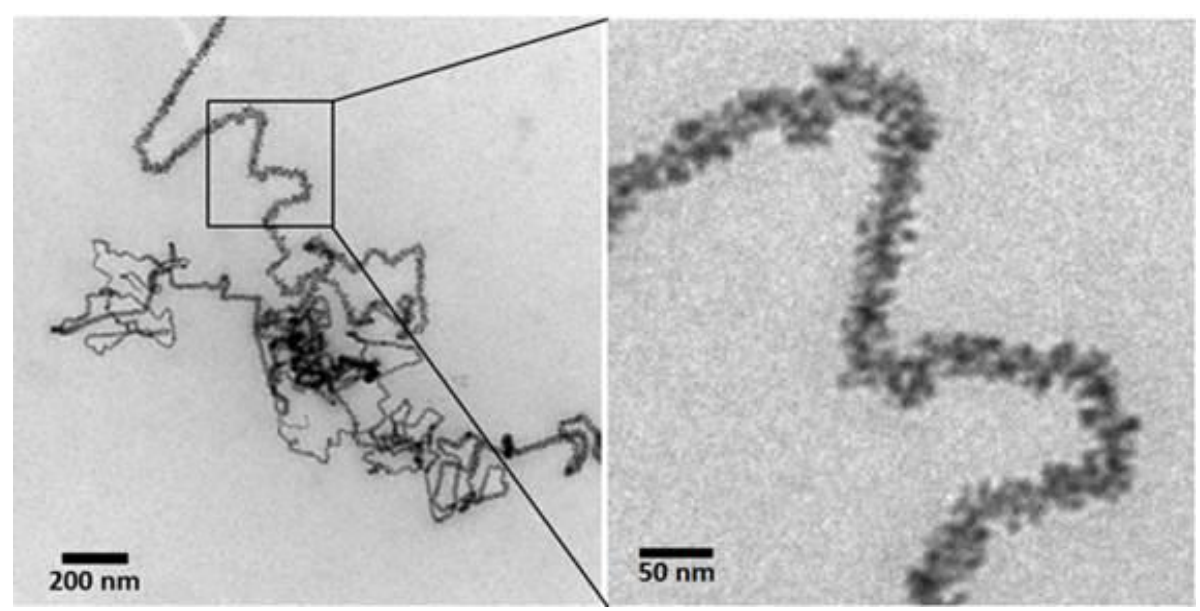

Figure S13. TEM images of P2tM-F 4 intermediate state (by cryofixation sample preparation). 


\section{Circular dichroism (CD)}

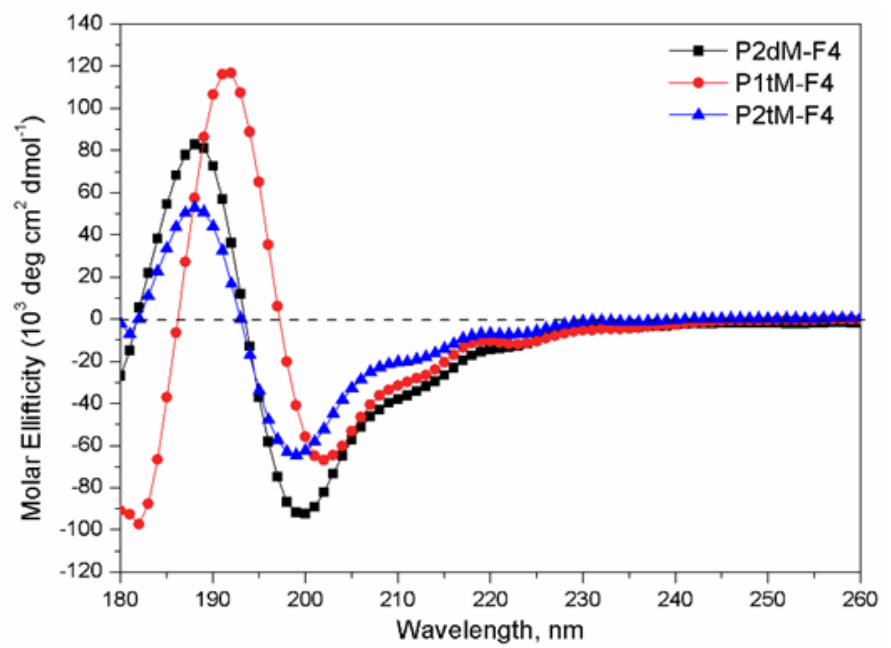

Figure S14. Circular dichroism (CD) spectra of the nanowires formed by $\mathbf{P 2 d M - F _ { 4 } , \mathbf { P 1 t M } - \mathbf { F } _ { \mathbf { 4 } } \text { and P2tM-F }}$

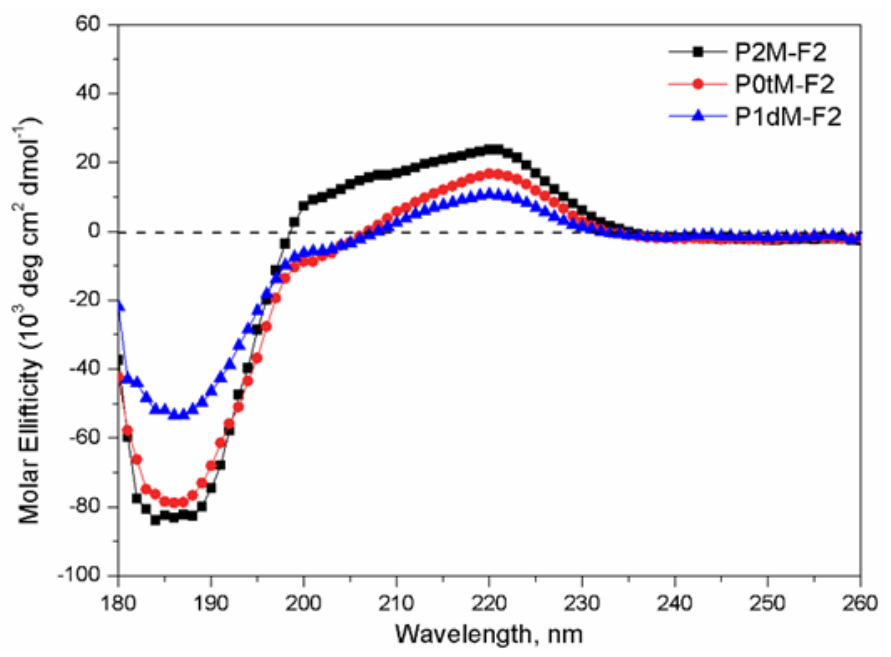

Figure S15. Circular dichroism (CD) spectra of the nanowires formed by P2M-F, P0tM-F 2 and P1tM-F

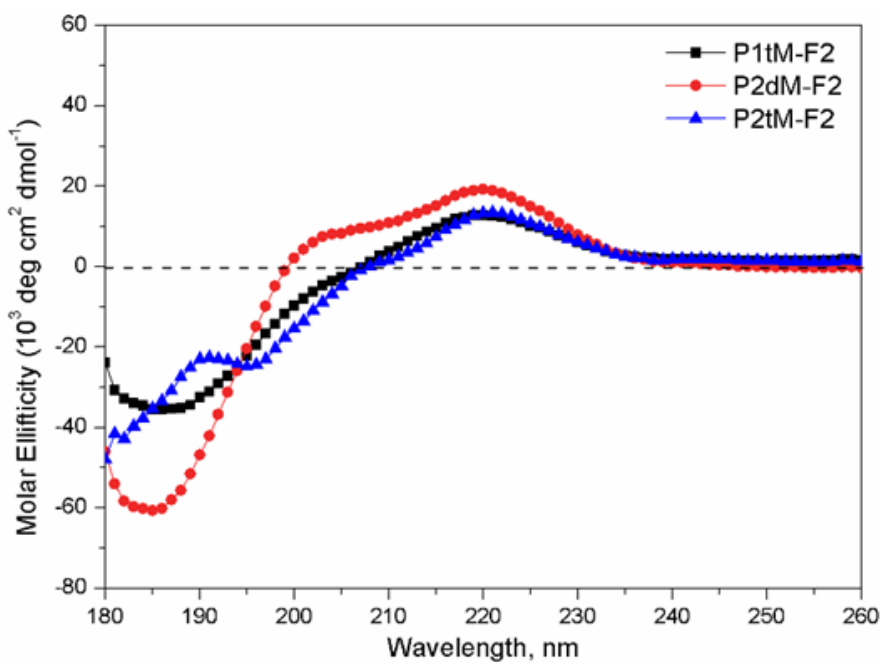

Figure S16. Circular dichroism (CD) spectra of the nanowires formed by $\mathbf{P 1 t M}-\mathbf{F}_{2}, \mathbf{P 2 d M}-\mathbf{F}_{2}$ and $\mathbf{P 2 t} \mathbf{M}-\mathbf{F}_{2}$ 


\section{Concentration dependent ELISA analysis}

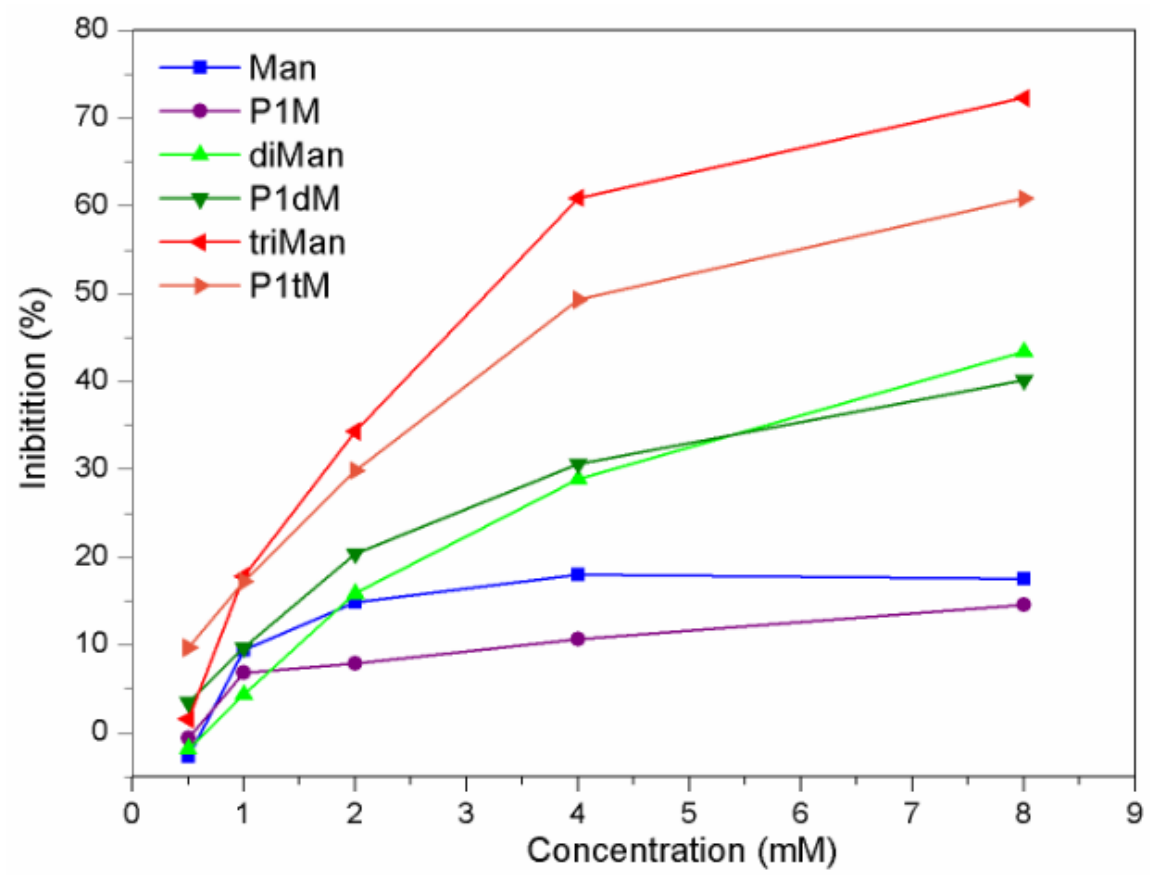

Figure S17. Concentration dependent ELISA analysis of glycoconjugates inhibition (\%) of 2G12 binding to gp120 coated in microtiter plates.

\section{Concentration dependent Agglutination}
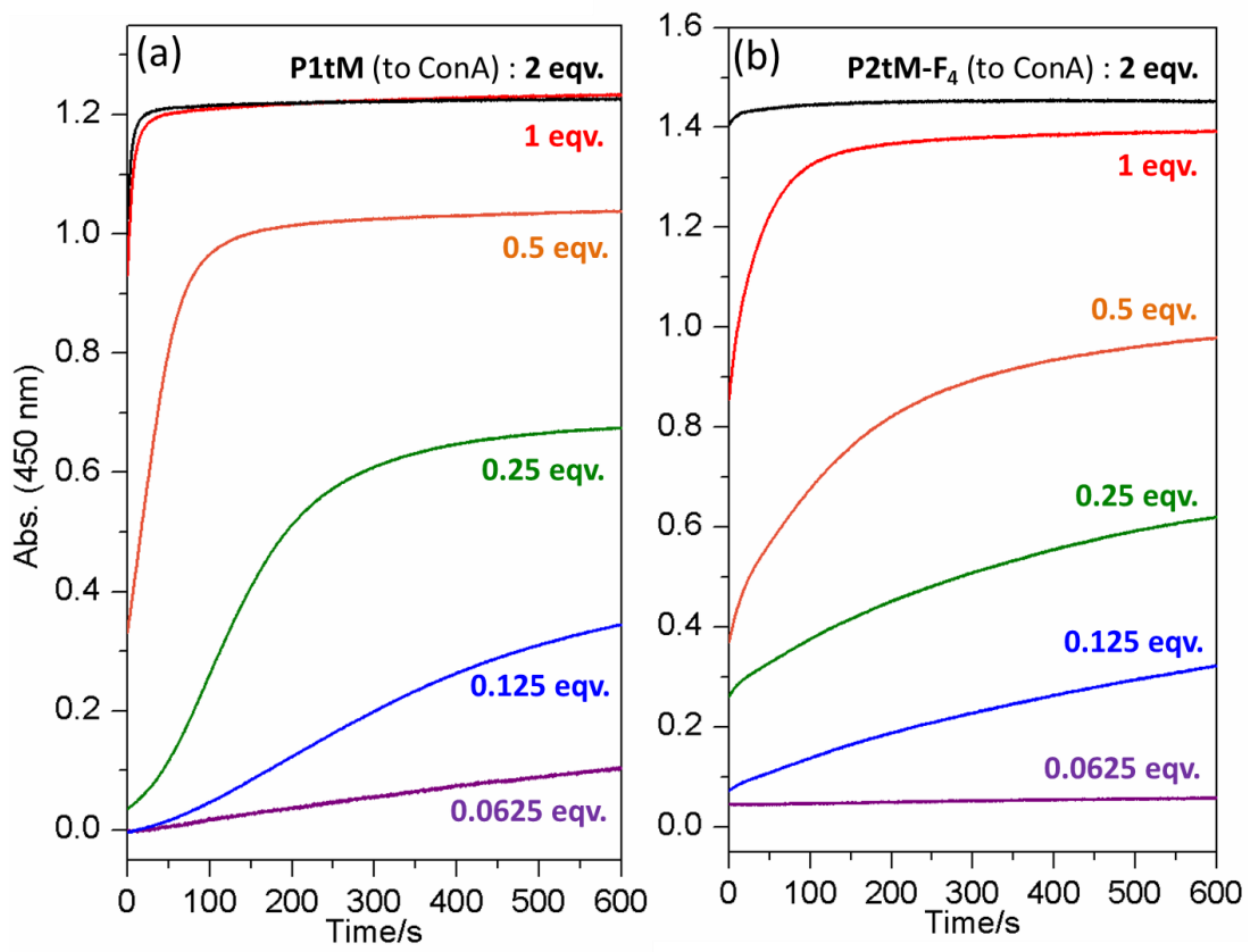

Figure S18. Concentration dependent Agglutination of Con A with glyco-dendronized polypeptide. 


\section{Synthesis and Characterization}

Synthesis of 1 (M0). Solution of Boc-Glu-OH (2.00 g, $8.09 \mathrm{mmol})$, 1-(3-Dimethylaminopropyl)3-ethylcarbodiimide hydrochloride (EDC) $(3.72 \mathrm{~g}, 19.4 \mathrm{mmol})$ in $50 \mathrm{~mL}$ of anhydrous dichloromethane (DCM) was cooled to $0^{\circ} \mathrm{C}$ in ice bath, after which pentafluorophenol (PFp-OH, $3.26 \mathrm{~g}, 17.7 \mathrm{mmol}$ ) dissolved in $5 \mathrm{~mL}$ DCM was added. The solution was allowed to warm to room temperature and stirred for another $5 \mathrm{~h}$. After the solvent was removed under reduced pressure, the crude product was purified by silica gel chromatography (200 300 mesh, DCM) to give $4.45 \mathrm{~g}$ (96\%) M0.

${ }^{1} \mathrm{H}$ NMR $\left(400 \mathrm{MHz}, \mathrm{CDCl}_{3}\right) \delta 5.18(\mathrm{~d}, J=8.3 \mathrm{~Hz}, 1 \mathrm{H}), 4.81(\mathrm{~s}, 1 \mathrm{H}), 2.93(\mathrm{dd}, J=14.7,7.2 \mathrm{~Hz}, 2 \mathrm{H}), 2.55(\mathrm{dd}$, $J=13.2,6.2 \mathrm{~Hz}, 1 \mathrm{H}), 2.29(\mathrm{td}, J=14.6,8.0 \mathrm{~Hz}, 1 \mathrm{H}), 1.50(\mathrm{~s}, 9 \mathrm{H})$.

${ }^{13} \mathrm{C}$ NMR $\left(400 \mathrm{MHz}, \mathrm{CDCl}_{3}\right) \delta 168.36,142.27,139.71,138.72,137.75,136.46,135.79,109.99,109.99,81.14$, $52.62,29.33,28.16,27.18$

$\left[\mathrm{C}_{22} \mathrm{H}_{15} \mathrm{~F}_{10} \mathrm{NO}_{6}+\mathrm{Na}\right]^{+}=602.35$; found 602.45

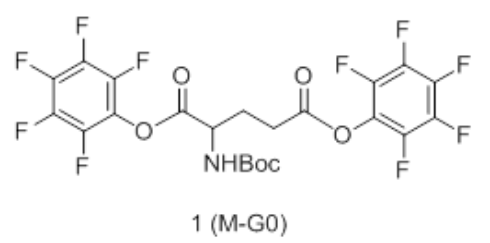

Synthesis of 2. Solution of Boc-Lys(Boc)-OH (3.00 g, $8.66 \mathrm{mmol})$, EDC (2.00 g, $10.4 \mathrm{mmol})$, Hydroxybenzotriazole (HOBT) $(1.41 \mathrm{~g}, 10.4 \mathrm{mmol})$ in $50 \mathrm{~mL}$ of anhydrous $\mathrm{DCM}$ was cooled to $0^{\circ} \mathrm{C}$ in ice bath. To this solution N,N-Diisopropylethylamine (DIPEA) $(4.47 \mathrm{~mL}, 26.0 \mathrm{mmol})$ was slowly added, and then $\mathrm{H}-\mathrm{Glu}(\mathrm{OMe})-\mathrm{OMe} \cdot \mathrm{HCl}(2.01 \mathrm{~g}, 9.56 \mathrm{mmol})$. The solution was allowed to warm to room temperature and stirred overnight. Then the solution was washed with $10 \mathrm{~mL} 2 \mathrm{M} \mathrm{HCl}$. The organic layer was dried over $\mathrm{MgSO}_{4}$, and the solvent was removed under reduced pressure. The crude product was purified by silica gel chromatography (200 300 mesh, 2:1 ethyl acetate: hexane) to give $3.76 \mathrm{~g}(86 \%) 2$.

${ }^{1} \mathrm{H}$ NMR $\left(400 \mathrm{MHz}, \mathrm{CDCl}_{3}\right) \delta 6.85(\mathrm{~d}, J=7.5 \mathrm{~Hz}, 1 \mathrm{H}), 5.18(\mathrm{~s}, 1 \mathrm{H}), 4.86-4.46(\mathrm{~m}, 2 \mathrm{H}), 4.09(\mathrm{~d}, J=5.8 \mathrm{~Hz}$, 1H), 3.77 (s, 3H), 3.69 (s, 3H), $3.13(\mathrm{dd}, J=11.5,5.7 \mathrm{~Hz}, 2 \mathrm{H}), 2.54-2.32$ (m, 2H), 2.24 (dtd, $J=12.5,7.5$, $5.1 \mathrm{~Hz}, 1 \mathrm{H}), 2.02(\mathrm{dtd}, J=14.4,8.1,6.5 \mathrm{~Hz}, 1 \mathrm{H}), 1.92-1.77(\mathrm{~m}, 2 \mathrm{H}), 1.74-1.17(\mathrm{~m}, 23 \mathrm{H})$.

${ }^{13} \mathrm{C}$ NMR $\left(400 \mathrm{MHz}, \mathrm{CDCl}_{3}\right) \delta 173.19,171.99,156.15,79.96,79.01,77.38,54.32,52.49,51.80,51.54,39.83$, 31.87, 29.93, 29.54, 28.41, 28.27, 27.09, 22.43.

$\left[\mathrm{C}_{23} \mathrm{H}_{41} \mathrm{~N}_{3} \mathrm{O}_{9}+\mathrm{Na}\right]^{+}=526.60$; found 526.47

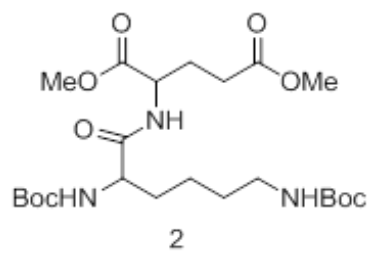

Synthesis of $3.2(3.76 \mathrm{~g}, 7.48 \mathrm{mmol})$ was dissolved in $20 \mathrm{~mL}$ methanol, then $10 \mathrm{~mL} \mathrm{H} \mathrm{H}_{2} \mathrm{O}$ was added and cooled to $0^{\circ} \mathrm{C}$. To this solution, $\mathrm{LiOH} \cdot \mathrm{H}_{2} \mathrm{O}(3.14 \mathrm{~g}, 74.8 \mathrm{mmol})$ was added. The mixture was allowed to warm to room temperature and stirred for $4 \mathrm{~h}$. The solution was neutralized by adding $2 \mathrm{M} \mathrm{HCl}$ until $\mathrm{pH} 3 \sim 4$. The methanol was removed under reduced pressure, and the aqueous solution was extracted with ethyl acetate (EA) 
(30 $\mathrm{mL} \times 3$ ). The organic layers was combined and dried over $\mathrm{MgSO}_{4}$. The solvent was removed under reduced pressure to give $3.27 \mathrm{~g}$ (92\%) 2a, which do not need further purification.

${ }^{1} \mathrm{H}$ NMR (400 MHz, DMSO) $\delta 12.41(\mathrm{~s}, 2 \mathrm{H}), 7.99(\mathrm{~d}, J=7.8 \mathrm{~Hz}, 1 \mathrm{H}), 7.05-6.68(\mathrm{~m}, 2 \mathrm{H}), 4.22(\mathrm{td}, J=8.9$, $5.0 \mathrm{~Hz}, 1 \mathrm{H}), 3.89(\mathrm{~d}, J=4.8 \mathrm{~Hz}, 1 \mathrm{H}), 3.03-2.75(\mathrm{~m}, 2 \mathrm{H}), 2.28(\mathrm{t}, J=7.7 \mathrm{~Hz}, 2 \mathrm{H}), 2.08-1.88(\mathrm{~m}, 1 \mathrm{H}), 1.77$ (dd, $J=13.5,7.9 \mathrm{~Hz}, 1 \mathrm{H}), 1.67-1.04(\mathrm{~m}, 23 \mathrm{H})$.

${ }^{13} \mathrm{C}$ NMR (400 MHz, MeOD) $\delta 174.95,173.90,173.16,171.58,157.15,156.44,79.22,78.46,60.15,54.56$, $51.42,39.64,31.47,29.63,29.18,27.44,27.36,26.62,22.68$

$\left[\mathrm{C}_{21} \mathrm{H}_{37} \mathrm{~N}_{3} \mathrm{O}_{9}+\mathrm{Na}\right]^{+}=498.54 ;$ found 498.40

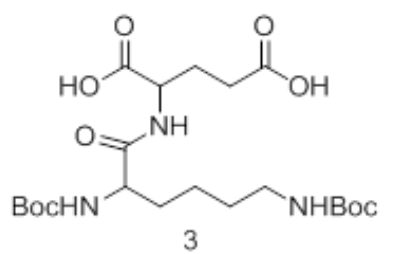

Synthesis of 4 (M1). Solution of 3 (3.27 g, $6.88 \mathrm{mmol})$, EDC (3.04 g, $16.5 \mathrm{mmol})$ in $50 \mathrm{~mL}$ of anhydrous DCM was cooled $0^{\circ} \mathrm{C}$ in ice bath, after which Pentafluorophenol (PFp-OH, $\left.2.78 \mathrm{~g}, 15.1 \mathrm{mmol}\right)$ dissolved in 5 $\mathrm{mL}$ DCM was added. The solution was allowed to warm to room temperature and stirred for another $5 \mathrm{~h}$. After which the solution was washed with $10 \mathrm{~mL} \mathrm{H}_{2} \mathrm{O}$. The organic layer was dried over $\mathrm{MgSO}_{4}$, and the solvent was removed under reduced pressure. The crude product was purified by silica gel chromatography (200 300 mesh, 2:1 ethyl acetate: hexane) to give $5.00 \mathrm{~g}(90 \%) 4$ (M1).

${ }^{1} \mathrm{H}$ NMR $\left(400 \mathrm{MHz}, \mathrm{CDCl}_{3}\right) \delta 7.25(\mathrm{~d}, J=11.9 \mathrm{~Hz}, 1 \mathrm{H}), 5.52-5.20(\mathrm{~m}, 1 \mathrm{H}), 5.07(\mathrm{tt}, J=8.6,4.3 \mathrm{~Hz}, 2 \mathrm{H})$, $4.70(\mathrm{~s}, 2 \mathrm{H}), 4.27-4.00(\mathrm{~m}, 2 \mathrm{H}), 3.26-3.02(\mathrm{~m}, 3 \mathrm{H}), 3.00-2.82(\mathrm{~m}, 3 \mathrm{H}), 2.58(\mathrm{ddd}, J=21.9,7.8,5.1 \mathrm{~Hz}$, $2 \mathrm{H}), 2.40-2.23(\mathrm{~m}, 2 \mathrm{H}), 1.98-1.82(\mathrm{~m}, 2 \mathrm{H}), 1.79-1.63(\mathrm{~m}, 2 \mathrm{H}), 1.45(\mathrm{~d}, J=2.6 \mathrm{~Hz}, 36 \mathrm{H})$.

13C NMR (400 MHz, CDCl3) $\delta$ 174.73, 173.21, 168.32, 167.64, 156.40, 156.13, 142.22, 140.82, 139.71, $139.13,138.29,136.63,80.38,79.35,60.46,54.36,51.00,50.96,39.59,39.46,31.22,29.61,29.42,29.13$, $28.29,28.12,28.10,26.73,26.68,22.42,20.63,14.07$.

$\left[\mathrm{C}_{33} \mathrm{H}_{35} \mathrm{~F}_{10} \mathrm{~N}_{3} \mathrm{O}_{9}+\mathrm{Na}\right]^{+}=830.64$; found 830.38

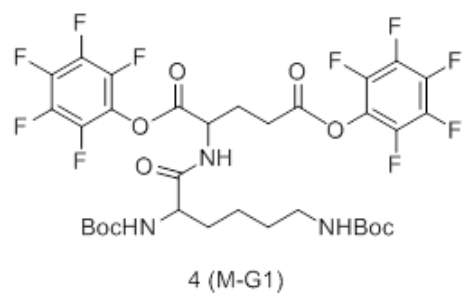

Synthesis of $5.2(3.76 \mathrm{~g}, 7.48 \mathrm{mmol})$ was cooled to $0^{\circ} \mathrm{C}, 20 \mathrm{~mL}$ of $4 \mathrm{M} \mathrm{HCl}$ solution in ethyl acetate was added. The mixture was kept at room temperature for further 20 min while white precipitates formed. The precipitate was filtered and washed with ethyl acetate several times to give $2.79 \mathrm{~g}(98 \%) \mathbf{5}$.

${ }^{1} \mathrm{H}$ NMR (400 MHz, $\left.\mathrm{D}_{2} \mathrm{O}\right) \delta 4.46(\mathrm{dd}, J=8.7,5.7 \mathrm{~Hz}, 1 \mathrm{H}), 4.02(\mathrm{t}, J=6.5 \mathrm{~Hz}, 1 \mathrm{H}), 3.70(\mathrm{~s}, 3 \mathrm{H}), 3.64(\mathrm{~s}, 2 \mathrm{H})$, $2.97(\mathrm{t}, J=7.7 \mathrm{~Hz}, 2 \mathrm{H}), 2.58-2.36(\mathrm{~m}, 2 \mathrm{H}), 2.19(\mathrm{qd}, J=7.5,5.7 \mathrm{~Hz}, 1 \mathrm{H}), 2.07-1.82(\mathrm{~m}, 3 \mathrm{H}), 1.68(\mathrm{dt}, J=$ 15.6, 7.7 Hz, 2H), $1.44(\mathrm{qd}, J=7.7,3.2 \mathrm{~Hz}, 2 \mathrm{H})$.

${ }^{13} \mathrm{C}$ NMR $\left(400 \mathrm{MHz}, \mathrm{D}_{2} \mathrm{O}\right) \delta 175.49,173.17,169.83,53.05,52.77,52.36,52.17,39.02,30.32,29.83,26.35$, $25.43,20.96$ 
$\left[\mathrm{C}_{13} \mathrm{H}_{25} \mathrm{~N}_{3} \mathrm{O}_{5}+\mathrm{Na}\right]^{+}=326.35 ;$ found 326.25

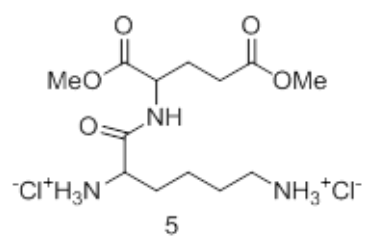

Synthesis of 6. Solution of Boc-Lys(Boc)-OH (3.00 g, $8.66 \mathrm{mmol})$, EDC (2.00 g, $10.4 \mathrm{mmol})$, HOBT (1.41 g, $10.4 \mathrm{mmol}$ ) in $50 \mathrm{~mL}$ of anhydrous DCM was cooled to $0^{\circ} \mathrm{C}$ in ice bath. To this solution DIPEA (4.47 mL, $26.0 \mathrm{mmol}$ ) was slowly added, and then $5(1.63 \mathrm{~g}, 4.33 \mathrm{mmol})$. The solution was allowed to warm to room temperature and stirred overnight. Then the solution was washed with $10 \mathrm{~mL} 2 \mathrm{M} \mathrm{HCl}$. The organic layer was dried over $\mathrm{MgSO}_{4}$, and the solvent was removed under reduced pressure. The crude product was purified by silica gel chromatography (200 300 mesh, ethyl acetate) to give $3.41 \mathrm{~g}(82 \%) 6$.

${ }^{1} \mathrm{H}$ NMR $\left(400 \mathrm{MHz}, \mathrm{CDCl}_{3}\right) \delta 7.44(\mathrm{~s}, 2 \mathrm{H}), 7.23(\mathrm{~s}, 1 \mathrm{H}), 6.15-5.45(\mathrm{~m}, 2 \mathrm{H}), 4.93(\mathrm{~d}, J=73.2 \mathrm{~Hz}, 2 \mathrm{H}), 4.68$ $-4.17(\mathrm{~m}, 4 \mathrm{H}), 3.75(\mathrm{~s}, 3 \mathrm{H}), 3.67(\mathrm{~s}, 3 \mathrm{H}), 3.06(\mathrm{t}, J=26.3 \mathrm{~Hz}, 5 \mathrm{H}), 2.42(\mathrm{dd}, J=10.1,5.6 \mathrm{~Hz}, 2 \mathrm{H}), 2.28-$ $2.12(\mathrm{~m}, 1 \mathrm{H}), 2.09-1.93(\mathrm{~m}, 2 \mathrm{H}), 1.85-1.63(\mathrm{~m}, 6 \mathrm{H}), 1.58-1.31(\mathrm{~m}, 51 \mathrm{H})$.

${ }^{13} \mathrm{C}$ NMR $\left(101 \mathrm{MHz}, \mathrm{CDCl}_{3}\right) \delta 173.63,173.22,172.26,171.86,156.20,79.90,52.49,51.82,51.62,40.03$, $30.02,29.53,29.46,28.43,28.40,28.36,24.73,22.87,22.69,21.04,14.18,12.14$

$\left[\mathrm{C}_{45} \mathrm{H}_{81} \mathrm{~N}_{7} \mathrm{O}_{15}+\mathrm{Na}\right]^{+}=983.17$; found 982.67

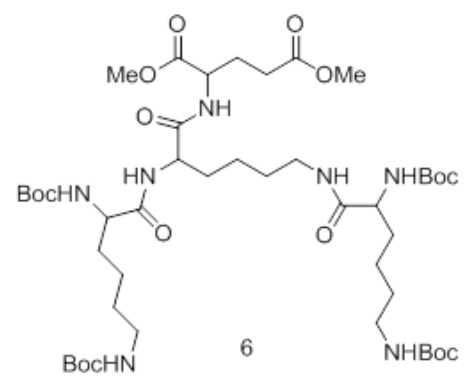

Synthesis of 7.6 ( $3.41 \mathrm{~g}, 3.55 \mathrm{mmol}$ ) was dissolved in $20 \mathrm{~mL}$ methanol, then $10 \mathrm{~mL} \mathrm{H}_{2} \mathrm{O}$ was added and cooled to $0^{\circ} \mathrm{C}$. To this solution, $\mathrm{LiOH} \cdot \mathrm{H}_{2} \mathrm{O}(1.49 \mathrm{~g}, 35.5 \mathrm{mmol})$ was added. The mixture was allowed to warm to room temperature and stirred for $4 \mathrm{~h}$. The solution was neutralized by adding $2 \mathrm{M} \mathrm{HCl} \mathrm{until} \mathrm{pH} 3 \sim 4$. The methanol was removed under reduced pressure, and the aqueous solution was extracted with EA ( $30 \mathrm{~mL} \times 3)$. The organic layers was combined and dried over $\mathrm{MgSO}_{4}$. The solvent was removed under reduced pressure to give $3.14 \mathrm{~g}(95 \%) 7$, which do not need further purification.

${ }^{1} \mathrm{H}$ NMR $\left(400 \mathrm{MHz}, \mathrm{CDCl}_{3}\right) \delta 7.66(\mathrm{~s}, 3 \mathrm{H}), 6.06(\mathrm{dd}, J=155.3,89.9 \mathrm{~Hz}, 2 \mathrm{H}), 5.00(\mathrm{~d}, J=71.9 \mathrm{~Hz}, 1 \mathrm{H}), 4.74$ $-4.13(\mathrm{~m}, 4 \mathrm{H}), 3.54-2.90(\mathrm{~m}, 6 \mathrm{H}), 2.49$ (s, 2H), 2.29 (d, $J=6.7 \mathrm{~Hz}, 1 \mathrm{H}), 1.59$ (dd, $J=116.3,27.8 \mathrm{~Hz}, 58 \mathrm{H})$.

${ }^{13} \mathrm{C}$ NMR $\left(400 \mathrm{MHz}, \mathrm{CDCl}_{3}\right.$ ) $\delta$ 175.94, 174.82, 173.46, 172.26, 156.52, 80.23, 80.03, 79.40, 60.44, 54.23, $51.81,41.18,40.20,38.66,32.47,32.12,28.42,28.37,22.59,14.19$

$\left[\mathrm{C}_{43} \mathrm{H}_{77} \mathrm{~N}_{7} \mathrm{O}_{15}+\mathrm{Na}\right]^{+}=955.12$; found 954.53 


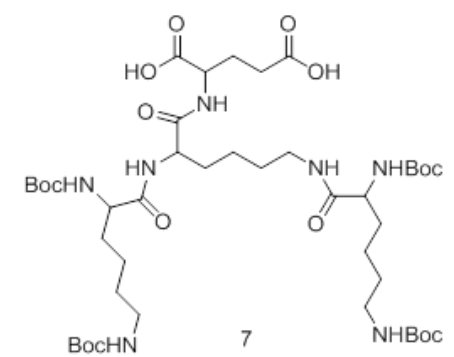

Synthesis of 8 (M2). Solution of 7 (3.14 g, $3.37 \mathrm{mmol})$, EDC (1.55 g, $8.09 \mathrm{mmol})$ in $20 \mathrm{~mL}$ of anhydrous DCM was cooled $0^{\circ} \mathrm{C}$ in ice bath, after which Pentafluorophenol (PFp-OH, $1.36 \mathrm{~g}, 7.42 \mathrm{mmol}$ ) dissolved in 2 $\mathrm{mL}$ DCM was added. The solution was allowed to warm to room temperature and stirred for another $5 \mathrm{~h}$. After which the solution was washed with $5 \mathrm{~mL} \mathrm{H}_{2} \mathrm{O}$. The organic layer was dried over $\mathrm{MgSO}_{4}$, and the solvent was removed under reduced pressure. The crude product was purified by silica gel chromatography (200 300 mesh, ethyl acetate) to give $3.62 \mathrm{~g}(85 \%) 8$ (M2).

${ }^{1} \mathrm{H}$ NMR (400 MHz, $\left.\mathrm{CDCl}_{3}\right) \delta 7.65(\mathrm{t}, J=158.0 \mathrm{~Hz}, 2 \mathrm{H}), 6.12-5.35(\mathrm{~m}, 2 \mathrm{H}), 4.92(\mathrm{~d}, J=67.6 \mathrm{~Hz}, 3 \mathrm{H}), 4.29$ $(\mathrm{d}, J=52.2 \mathrm{~Hz}, 3 \mathrm{H}), 3.56(\mathrm{~s}, 1 \mathrm{H}), 3.27-2.81(\mathrm{~m}, 7 \mathrm{H}), 2.55(\mathrm{td}, J=13.3,7.8 \mathrm{~Hz}, 1 \mathrm{H}), 2.43-2.24(\mathrm{~m}, 1 \mathrm{H})$, $1.95-1.07(\mathrm{~m}, 58 \mathrm{H})$.

${ }^{13} \mathrm{C}$ NMR $\left(400 \mathrm{MHz}, \mathrm{CDCl}_{3}\right) \delta 174.15,173.56,172.59,168.52,167.80,156.55,156.37,156.22,142.27$, 139.76, 139.11, 136.62, 80.21, 80.04, 79.12, 54.20, 53.39, 51.22, 51.14, 40.17, 39.70, 38.53, 32.41, 29.45, $28.34,28.24,28.20,22.82,22.60,20.96,14.11$.

$\left[\mathrm{C}_{55} \mathrm{H}_{75} \mathrm{~F}_{10} \mathrm{~N}_{7} \mathrm{O}_{15}+\mathrm{Na}\right]^{+}=1287.22 ;$ found 1286.60

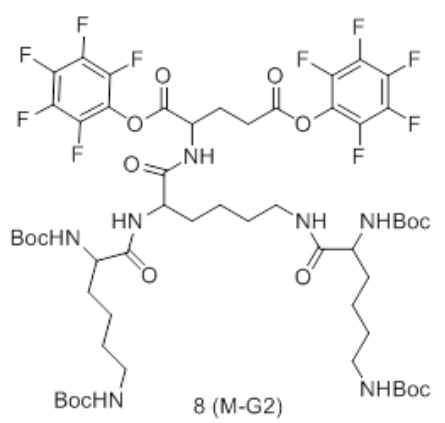

Synthesis of 9. Solution of Boc-Lys(Boc)-OH (3.00 g, $8.66 \mathrm{mmol})$, EDC (2.00 g, $10.4 \mathrm{mmol})$, HOBT (1.41 g, $10.4 \mathrm{mmol}$ ) in $50 \mathrm{~mL}$ of anhydrous DCM was cooled to $0^{\circ} \mathrm{C}$ in ice bath. To this solution DIPEA (4.47 $\mathrm{mL}$, $26.0 \mathrm{mmol})$ was slowly added, and then 2-Propynylamine $(0.48 \mathrm{~g}, 8.72 \mathrm{mmol})$. The solution was allowed to warm to room temperature and stirred overnight. Then the solution was washed with $10 \mathrm{~mL} 2 \mathrm{M} \mathrm{HCl}$. The organic layer was dried over $\mathrm{MgSO}_{4}$, and the solvent was removed under reduced pressure. The crude product was purified by silica gel chromatography (200 300 mesh, 2:1 ethyl acetate: hexane) to give $2.98 \mathrm{~g} \mathrm{(91 \% )} 9$. ${ }^{1} \mathrm{H}$ NMR (400 MHz, $\left.\mathrm{CDCl}_{3}\right) \delta 6.73(\mathrm{~s}, 1 \mathrm{H}), 5.23(\mathrm{~s}, 1 \mathrm{H}), 4.68(\mathrm{~s}, 1 \mathrm{H}), 4.10(\mathrm{~s}, 1 \mathrm{H}), 4.05$ (dd, J = 5.1, $2.4 \mathrm{~Hz}$, 2H), $3.12(\mathrm{~d}, J=6.2 \mathrm{~Hz}, 2 \mathrm{H}), 2.24(\mathrm{t}, J=2.5 \mathrm{~Hz}, 1 \mathrm{H}), 1.85(\mathrm{ddd}, J=13.8,8.9,6.0 \mathrm{~Hz}, 1 \mathrm{H}), 1.74-1.59(\mathrm{~m}$, $1 \mathrm{H}), 1.57-1.35(\mathrm{~m}, 23 \mathrm{H})$.

${ }^{13} \mathrm{C}$ NMR $\left(101 \mathrm{MHz}, \mathrm{CDCl}_{3}\right) \delta 156.18,79.34,71.61,54.24,39.87,31.87,29.65,29.09,28.45,28.33,22.56$ $\left[\mathrm{C}_{19} \mathrm{H}_{33} \mathrm{~N}_{3} \mathrm{O}_{5}+\mathrm{Na}\right]^{+}=406.49$; found 406.32 


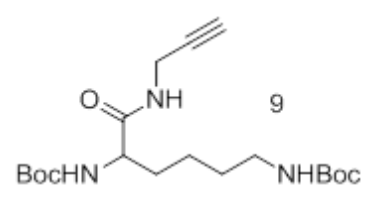

Synthesis of 10 (M-a). $9(2.98 \mathrm{~g}, 7.80 \mathrm{mmol})$ was cooled to $0^{\circ} \mathrm{C}, 20 \mathrm{~mL}$ of $4 \mathrm{M} \mathrm{HCl}$ solution in ethyl acetate was added. The mixture was kept at room temperature for further 20 min while white precipitates formed. The precipitate was filtered and washed with ethyl acetate several times to give $1.98 \mathrm{~g}(98 \%) \mathbf{1 0}$ (M-a).

${ }^{1} \mathrm{H}$ NMR $\left(400 \mathrm{MHz}, \mathrm{D}_{2} \mathrm{O}\right) \delta 4.05-3.88(\mathrm{~m}, 1 \mathrm{H}), 3.03-2.86(\mathrm{~m}, 1 \mathrm{H}), 2.58(\mathrm{t}, J=2.5 \mathrm{~Hz}, 1 \mathrm{H}), 1.87(\mathrm{ddd}, J=$ $12.0,8.5,4.1 \mathrm{~Hz}, 1 \mathrm{H}), 1.65(\mathrm{dt}, J=15.5,7.7 \mathrm{~Hz}, 1 \mathrm{H}), 1.40(\mathrm{tt}, J=10.4,6.6 \mathrm{~Hz}, 1 \mathrm{H})$.

${ }^{13} \mathrm{C}$ NMR $\left(101 \mathrm{MHz}, \mathrm{D}_{2} \mathrm{O}\right) \delta 169.41,78.95,72.18,52.93,39.00,30.22,29.61,28.91,26.31,21.18,13.26$

$\left[\mathrm{C}_{9} \mathrm{H}_{17} \mathrm{~N}_{3} \mathrm{O}+\mathrm{Na}\right]^{+}=206.26$; found 206.19

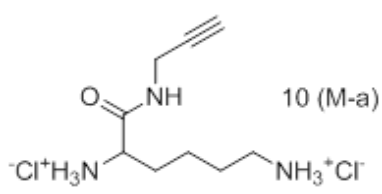

Synthesis of 11 (M-b). Solution of (Boc-Aminooxy)Acetic Acid (2 g, $10.5 \mathrm{mmol})$, EDC (2.41 g, $12.6 \mathrm{mmol})$ in $20 \mathrm{~mL}$ of anhydrous DCM was cooled $0^{\circ} \mathrm{C}$ in ice bath, after which Pentafluorophenol (PFp-OH, $2.13 \mathrm{~g}$, $11.6 \mathrm{mmol}$ ) dissolved in $5 \mathrm{~mL}$ DCM was added. The solution was allowed to warm to room temperature and stirred for another $5 \mathrm{~h}$, while white precipitates were formed. The solution was concentrate under reduced pressure and filtered to give $2.80 \mathrm{~g}(75 \%) \mathbf{1 1}$ (M-b).

${ }^{1} \mathrm{H}$ NMR (400 MHz, $\left.\mathrm{D}_{2} \mathrm{O}\right) \delta 4.83$ (s, 2H), $1.52(\mathrm{~s}, 9 \mathrm{H})$

${ }^{13} \mathrm{C}$ NMR $\left(400 \mathrm{MHz}, \mathrm{CDCl}_{3}\right) \delta 165.63,156.38,142.21,141.08,139.66,139.20,138.56,136.66,82.79,71.96$, 28.11 ,

$\left[\mathrm{C}_{13} \mathrm{H}_{12} \mathrm{~F}_{5} \mathrm{NO}_{5}+\mathrm{Na}\right]^{+}=380.24$; found 380.20

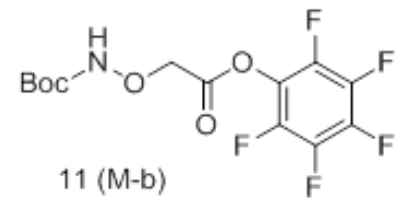

Synthesis of 12. Solution of azido butyric acid $(2.00 \mathrm{~g}, 15.5 \mathrm{mmol}$, the synthesis of azido butyric acid was reported elsewhere $\left.{ }^{3}\right)$, EDC $(3.57 \mathrm{~g}, 18.6 \mathrm{mmol})$ in $50 \mathrm{~mL}$ of anhydrous DCM was cooled $0^{\circ} \mathrm{C}$ in ice bath, after which Pentafluorophenol (PFp-OH, $3.15 \mathrm{~g}, 17.1 \mathrm{mmol}$ ) dissolved in $5 \mathrm{~mL}$ DCM was added. The solution was allowed to warm to room temperature and stirred for another $5 \mathrm{~h}$. After the solvent was removed under reduced pressure, the crude product was purified by silica gel chromatography (200 300 mesh, DCM) to give $4.11 \mathrm{~g}(90 \%) 12$.

${ }^{1} \mathrm{H}$ NMR $\left(400 \mathrm{MHz}, \mathrm{CDCl}_{3}\right) \delta 3.49(\mathrm{t}, J=6.5 \mathrm{~Hz}, 2 \mathrm{H}), 2.82(\mathrm{t}, J=7.2 \mathrm{~Hz}, 2 \mathrm{H}), 2.08(\mathrm{dq}, J=13.8,6.9 \mathrm{~Hz}$, $2 \mathrm{H})$.

${ }^{13} \mathrm{C}$ NMR $\left(400 \mathrm{MHz}, \mathrm{CDCl}_{3}\right) \delta 168.70,142.34,140.83,139.81,138.97,138.20,136.78,50.19,30.28,24.12$

$\left[\mathrm{C}_{10} \mathrm{H}_{6} \mathrm{~F}_{5} \mathrm{~N}_{3} \mathrm{O}_{2}+\mathrm{Na}\right]^{+}=318.18$; found 318.03 


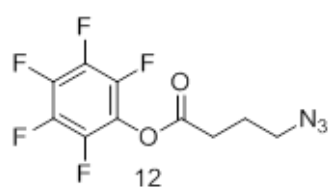

Synthesis of 13. Solution of Boc-Phe-OH (2.00 g, $7.55 \mathrm{mmol})$, EDC (1.74 g, $9.06 \mathrm{mmol})$, HOBT (1.22 g, $9.06 \mathrm{mmol}$ ) in $50 \mathrm{~mL}$ of anhydrous DCM was cooled to $0^{\circ} \mathrm{C}$ in ice bath. To this solution DIPEA (3.9 mL, 22.7 mmol) was slowly added, and then $\mathrm{H}-\mathrm{Phe}-\mathrm{OMe} \cdot \mathrm{HCl}(1.79 \mathrm{~g}, 8.3 \mathrm{mmol})$. The solution was allowed to warm to room temperature and stirred overnight. Then the solution was washed with $10 \mathrm{~mL} 2 \mathrm{M} \mathrm{HCl}$. The organic layer was dried over $\mathrm{MgSO}_{4}$, and the solvent was removed under reduced pressure. The crude product was purified by silica gel chromatography (200 300 mesh, 2:1 ethyl acetate: hexane) to give $3.06 \mathrm{~g}(96 \%) \mathbf{1 3}$.

${ }^{1} \mathrm{H}$ NMR $\left(400 \mathrm{MHz}, \mathrm{CDCl}_{3}\right) \delta 7.44-7.15(\mathrm{~m}, 8 \mathrm{H}), 7.09-6.93(\mathrm{~m}, 2 \mathrm{H}), 6.33(\mathrm{~d}, J=5.2 \mathrm{~Hz}, 1 \mathrm{H}), 4.84(\mathrm{dd}, J$ $=40.7,34.0 \mathrm{~Hz}, 2 \mathrm{H}), 4.35(\mathrm{~s}, 1 \mathrm{H}), 3.69(\mathrm{~s}, 3 \mathrm{H}), 3.07(\mathrm{dd}, J=8.1,6.0 \mathrm{~Hz}, 4 \mathrm{H}), 1.42(\mathrm{~s}, 9 \mathrm{H})$.

${ }^{13} \mathrm{C}$ NMR $\left(400 \mathrm{MHz}, \mathrm{CDCl}_{3}\right) \delta 171.35,170.77,136.49,135.64,129.37,129.25,128.68,128.55,127.20$, $126.99,55.69,53.72,53.27,52.26,38.27,37.97,28.23$

$\left[\mathrm{C}_{24} \mathrm{H}_{30} \mathrm{~N}_{2} \mathrm{O}_{5}+\mathrm{Na}\right]^{+}=449.51$; found 449.49

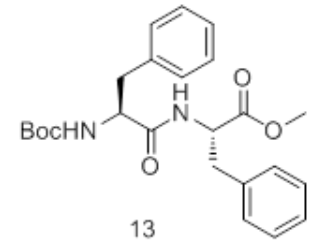

Synthesis of 14.13 (3.06 g, $7.17 \mathrm{mmol}$ ) was dissolved in $20 \mathrm{~mL}$ methanol, then $10 \mathrm{~mL} \mathrm{H}_{2} \mathrm{O}$ was added and cooled to $0^{\circ} \mathrm{C}$. To this solution, $\mathrm{LiOH} \cdot \mathrm{H}_{2} \mathrm{O}(3.01 \mathrm{~g}, 71.7 \mathrm{mmol})$ was added. The mixture was allowed to warm to room temperature and stirred for $4 \mathrm{~h}$. The solution was neutralized by adding $2 \mathrm{M} \mathrm{HCl}$ until $\mathrm{pH} \mathrm{3} \mathrm{4.} \mathrm{The}$ methanol was removed under reduced pressure, and the aqueous solution was extracted with EA ( $30 \mathrm{~mL} \times 3)$. The organic layers was combined and dried over $\mathrm{MgSO}_{4}$. The solvent was removed under reduced pressure to give $2.84 \mathrm{~g}(96 \%) \mathbf{1 4}$, which do not need further purification.

${ }^{1} \mathrm{H}$ NMR (400 MHz, DMSO) $\delta 8.11(\mathrm{~d}, J=7.8 \mathrm{~Hz}, 1 \mathrm{H}), 7.40-7.07(\mathrm{~m}, 10 \mathrm{H}), 6.91(\mathrm{~d}, J=8.8 \mathrm{~Hz}, 1 \mathrm{H}), 4.47$ $(\mathrm{td}, J=8.0,5.3 \mathrm{~Hz}, 1 \mathrm{H}), 4.17(\mathrm{td}, J=10.4,3.9 \mathrm{~Hz}, 1 \mathrm{H}), 3.10(\mathrm{dd}, J=13.8,5.1 \mathrm{~Hz}, 1 \mathrm{H}), 3.03-2.80(\mathrm{~m}, 2 \mathrm{H})$, $2.68(\mathrm{dd}, J=13.7,10.7 \mathrm{~Hz}, 1 \mathrm{H}), 1.21(\mathrm{~d}, J=59.3 \mathrm{~Hz}, 9 \mathrm{H})$.

${ }^{13} \mathrm{C}$ NMR $\left(400 \mathrm{MHz}, \mathrm{CDCl}_{3}\right) \delta 173.25,172.04,155.54,137.89,129.17,129.62,128.61,128.43,126.86$, $126.59,78.49,56.19,53.89,37.89,37.29,28.58$

$\left[\mathrm{C}_{23} \mathrm{H}_{28} \mathrm{~N}_{2} \mathrm{O}_{5}+\mathrm{Na}\right]^{+}=435.49$; found 435.45

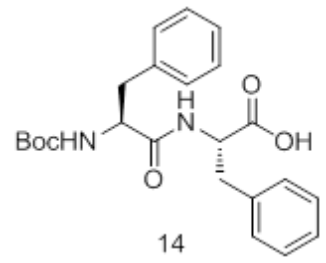

Synthesis of 15 Solution of $\mathbf{1 4}(2.84 \mathrm{~g}, 6.89 \mathrm{mmol})$, EDC (1.59 g, $8.27 \mathrm{mmol})$ in $20 \mathrm{~mL}$ of anhydrous DCM was cooled $0^{\circ} \mathrm{C}$ in ice bath, after which Pentafluorophenol (PFp-OH, $1.39 \mathrm{~g}, 7.58 \mathrm{mmol}$ ) dissolved in $2 \mathrm{~mL}$ DCM was added. The solution was allowed to warm to room temperature and stirred for another $5 \mathrm{~h}$. After the 
solvent was removed under reduced pressure, the crude product was purified by silica gel chromatography (200 300 mesh, DCM) to give $3.74 \mathrm{~g}(94 \%) 15$.

${ }^{1} \mathrm{H}$ NMR $\left(400 \mathrm{MHz}, \mathrm{CDCl}_{3}\right) \delta 7.52-6.89(\mathrm{~m}, 10 \mathrm{H}), 6.77-6.29(\mathrm{~m}, 1 \mathrm{H}), 5.26-4.87(\mathrm{~m}, 2 \mathrm{H}), 4.40(\mathrm{~s}, 1 \mathrm{H})$, $3.44-2.91(\mathrm{~m}, 4 \mathrm{H}), 1.41(\mathrm{~d}, J=6.2 \mathrm{~Hz}, 9 \mathrm{H})$.

${ }^{13} \mathrm{C}$ NMR $\left(400 \mathrm{MHz}, \mathrm{CDCl}_{3}\right) \delta 171.50,171.36,167.51,167.44,155.44,142.25,139.66,139.16,138.48$, 136.37, 136.34, 134.51, 134.42, 129.27, 129.15, 128.93, 128.85, 128.71, 127.62, 127.58, 127.08, 80.61, 55.67, 53.04, 52.87, 38.09, 37.96, 37.54, 37.40, 28.16

$\left[\mathrm{C}_{29} \mathrm{H}_{27} \mathrm{~F}_{5} \mathrm{~N}_{2} \mathrm{O}_{5}+\mathrm{Na}\right]^{+}=601.54$; found 601.52

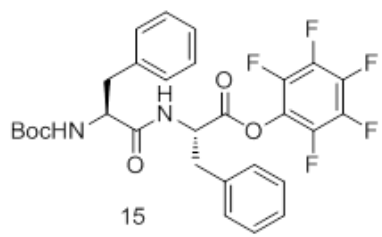

Synthesis of $16.13(3.06 \mathrm{~g}, 7.17 \mathrm{mmol})$ was cooled to $0^{\circ} \mathrm{C} ; 20 \mathrm{~mL}$ of $4 \mathrm{M} \mathrm{HCl}$ solution in ethyl acetate was added. The mixture was kept at room temperature for further 20 min while white precipitates formed. The precipitate was filtered and washed with ethyl acetate several times to give $2.59 \mathrm{~g} \mathrm{(98 \% )} \mathbf{1 6}$.

${ }^{1} \mathrm{H}$ NMR (400 MHz, DMSO) $\delta 9.07(\mathrm{~d}, J=7.6 \mathrm{~Hz}, 1 \mathrm{H}), 8.21(\mathrm{~s}, 3 \mathrm{H}), 7.49-7.12(\mathrm{~m}, 10 \mathrm{H}), 4.74-4.45$ (m, $1 \mathrm{H}), 4.07$ (dd, $J=7.7,5.5 \mathrm{~Hz}, 1 \mathrm{H}), 3.62$ (s, 3H), 3.03 (ddd, $J=21.6,14.5,6.6 \mathrm{~Hz}, 4 \mathrm{H})$.

${ }^{13} \mathrm{C}$ NMR $\left(400 \mathrm{MHz}, \mathrm{CDCl}_{3}\right) \delta 171.60,168.71,137.15,135.17,130.01,129.54,128.97,128.86,127.63$, $127.21,54.31,53.59,52.49,37.34,37.12$

$\left[\mathrm{C}_{19} \mathrm{H}_{22} \mathrm{~N}_{2} \mathrm{O}_{3}+\mathrm{Na}\right]^{+}=349.39$; found 349.37

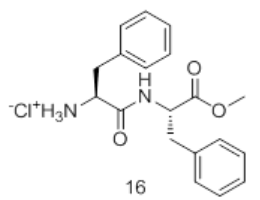

Synthesis of 17 (M-c). Solution of $16(1 \mathrm{~g}, 2.76 \mathrm{mmol})$ and DIPEA $(1.18 \mathrm{~mL}, 6.89 \mathrm{mmol})$ in $20 \mathrm{~mL}$ of anhydrous DCM was cooled to $0^{\circ} \mathrm{C}$ in ice bath, after which $12(0.82 \mathrm{~g}, 2.76 \mathrm{mmol})$ was added. The solution was allowed to warm to room temperature and stirred for another $5 \mathrm{~h}$. After the solvent was removed under reduced pressure, the crude product was purified by silica gel chromatography (200 300 mesh, 2:1 ethyl acetate: hexane) to give $1.11 \mathrm{~g}(92 \%) \mathbf{1 7}$ (M-c).

${ }^{1} \mathrm{H}$ NMR (400 MHz, DMSO) $\delta 8.47(\mathrm{~d}, J=7.5 \mathrm{~Hz}, 1 \mathrm{H}), 8.08(\mathrm{~d}, J=8.7 \mathrm{~Hz}, 1 \mathrm{H}), 7.43-7.10(\mathrm{~m}, 10 \mathrm{H}), 4.78-$ $4.38(\mathrm{~m}, 2 \mathrm{H}), 3.60(\mathrm{~s}, 3 \mathrm{H}), 2.92(\mathrm{dddd}, J=35.7,24.2,13.9,8.8 \mathrm{~Hz}, 7 \mathrm{H}), 2.08(\mathrm{t}, J=7.3 \mathrm{~Hz}, 2 \mathrm{H}), 1.60(\mathrm{dd}, J=$ $14.1,7.1 \mathrm{~Hz}, 2 \mathrm{H})$.

${ }^{13} \mathrm{C}$ NMR (101 MHz, DMSO) $\delta$ 172.20, 171.97, 171.41, 138.34, 137.50, 129.62, 129.52, 128.70, 128.39, $127.02,126.63,54.06,53.81,52.29,50.41,38.02,37.02,32.47,24.89$

$\left[\mathrm{C}_{23} \mathrm{H}_{27} \mathrm{~N}_{5} \mathrm{O}_{4}+\mathrm{Na}\right]^{+}=460.50$; found 460.45

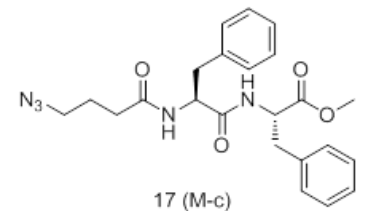


Synthesis of 18. Solution of $\mathbf{1 6}(1 \mathrm{~g}, 2.76 \mathrm{mmol})$ and DIPEA $(1.18 \mathrm{~mL}, 6.89 \mathrm{mmol})$ in $20 \mathrm{~mL}$ of anhydrous DCM was cooled to $0^{\circ} \mathrm{C}$ in ice bath, after which $15(1.60 \mathrm{~g}, 2.76 \mathrm{mmol})$ was added. White precipitates were formed immediately. The solution was allowed to warm to room temperature and stirred for another $5 \mathrm{~h}$ and the precipitates were filtered and washed with DCM for several times, to give $1.63 \mathrm{~g}(82 \%) \mathbf{1 8}$, which do not need further purification.

${ }^{1} \mathrm{H}$ NMR (400 MHz, DMSO) $\delta 8.54(\mathrm{dd}, J=59.1,7.8 \mathrm{~Hz}, 2 \mathrm{H}), 8.08(\mathrm{~d}, J=8.4 \mathrm{~Hz}, 1 \mathrm{H}), 7.18(\mathrm{dddd}, J=62.1$, 40.8, 24.2, $6.8 \mathrm{~Hz}, 19 \mathrm{H}), 6.64(\mathrm{~d}, J=8.7 \mathrm{~Hz}, 1 \mathrm{H}), 4.79-4.41(\mathrm{~m}, 3 \mathrm{H}), 4.15(\mathrm{~s}, 1 \mathrm{H}), 3.58(\mathrm{~s}, 3 \mathrm{H}), 3.17-2.89$ (m, 3H), $2.78-2.54(\mathrm{~m}, 3 \mathrm{H}), 2.39(\mathrm{t}, J=11.1 \mathrm{~Hz}, 2 \mathrm{H}), 1.17(\mathrm{~d}, J=67.1 \mathrm{~Hz}, 9 \mathrm{H})$.

${ }^{13} \mathrm{C}$ NMR $\left(400 \mathrm{MHz}, \mathrm{CDCl}_{3}\right) \delta 172.23,171.84,171.56,171.04,155.43,138.59,138.18,137.95,137.38$, $129.87,129.78,129.62,129.50,128.76,128.46,128.28,127.07,126.81,126.60,126.45,78.31,55.79,54.22$, $53.95,52.27,38.60,38.41,38.05,37.13,28.56$

$\left[\mathrm{C}_{42} \mathrm{H}_{48} \mathrm{~N}_{4} \mathrm{O}_{7}+\mathrm{Na}\right]^{+}=743.85$; found 743.71

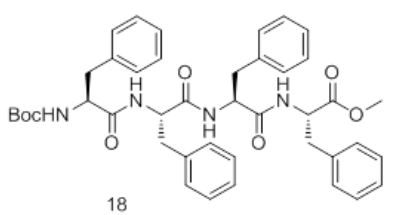

Synthesis of $19.18(1.63 \mathrm{~g}, 2.26 \mathrm{mmol})$ was cooled to $0^{\circ} \mathrm{C}$, after which $5 \mathrm{~mL}$ of trifluoroacetate (TFA) was added. The mixture was kept at room temperature for further $20 \mathrm{~min}$, then TAF was removed under reduced pressure. The syrup residue was dilute by $3 \mathrm{~mL}$ of methanol and precipitate in diethyl ether. The precipitate was washed by diethyl ether sever time and filtered to give $1.39 \mathrm{~g}(81 \%) \mathbf{1 9}$.

${ }^{1} \mathrm{H}$ NMR (400 MHz, DMSO) $\delta 9.07(\mathrm{~d}, J=7.6 \mathrm{~Hz}, 1 \mathrm{H}), 8.21(\mathrm{~s}, 3 \mathrm{H}), 7.49-7.12(\mathrm{~m}, 10 \mathrm{H}), 4.74-4.45(\mathrm{~m}$, $1 \mathrm{H}), 4.07$ (dd, $J=7.7,5.5 \mathrm{~Hz}, 1 \mathrm{H}), 3.62$ (s, 3H), 3.03 (ddd, $J=21.6,14.5,6.6 \mathrm{~Hz}, 4 \mathrm{H}$ ).

${ }^{13} \mathrm{C}$ NMR (101 MHz, DMSO) $\delta 172.10,171.45,170.79,168.26,137.97,137.82,137.47,135.10,130.02$, $129.71,129.62,129.49,128.91,128.73,128.53,128.49,127.55,127.02,126.80,126.70,54.35,54.05,53.46$, $52.31,38.13,37.44,37.09$.

$\left[\mathrm{C}_{37} \mathrm{H}_{40} \mathrm{~N}_{4} \mathrm{O}_{5}+\mathrm{Na}\right]^{+}=643.74 ;$ found 643.67

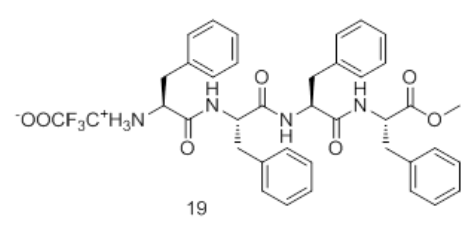

Synthesis of 20 (M-d). 19 (1.39 g, $1.81 \mathrm{mmol})$ and DIPEA (0.93 mL, $5.43 \mathrm{mmol})$ was dissolved in $3 \mathrm{~mL}$ N,N-Dimethylformamide (DMF). After which $12(0.47 \mathrm{~g}, 1.81 \mathrm{mmol})$ was added, and white precipitate formed immediately. The mixture was allowed to stirred under room temperature for another $2 \mathrm{~h}$, and precipitate in diethyl ether and filtered to give $0.99 \mathrm{~g}(76 \%) \mathbf{2 0}$ (M-d).

${ }^{1} \mathrm{H}$ NMR (400 MHz, DMSO) $\delta 8.59(\mathrm{~d}, J=7.5 \mathrm{~Hz}, 1 \mathrm{H}), 8.22(\mathrm{~d}, J=8.4 \mathrm{~Hz}, 1 \mathrm{H}), 8.12(\mathrm{~d}, J=8.7 \mathrm{~Hz}, 1 \mathrm{H})$, $8.06(\mathrm{~d}, J=8.4 \mathrm{~Hz}, 1 \mathrm{H}), 7.43-6.96(\mathrm{~m}, 20 \mathrm{H}), 4.63-4.34(\mathrm{~m}, 4 \mathrm{H}), 3.54(\mathrm{~s}, 3 \mathrm{H}), 3.11-2.56(\mathrm{~m}, 11 \mathrm{H}), 2.03(\mathrm{t}$, $J=7.2 \mathrm{~Hz}, 2 \mathrm{H}), 1.62-1.44(\mathrm{~m}, 2 \mathrm{H})$.

${ }^{13} \mathrm{C}$ NMR (400 MHz, $\left.\mathrm{CDCl}_{3}\right) \delta 172.08,171.48,171.04,138.52,138.11,137.69,137.52,129.72,129.62$, $129.54,128.72$, 128.45, 128.33, 127.02, 126.69, 126.60, 54.36, 54.26, 52.33, 50.37, 38.01, 37.90, 37.71, 37.02, $32.49,24.85$ 
$\left[\mathrm{C}_{41} \mathrm{H}_{45} \mathrm{~N}_{7} \mathrm{O}_{6}+\mathrm{Na}\right]^{+}=754.84$; found 754.70

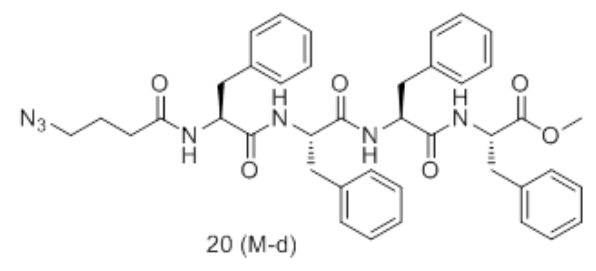

\section{General method of polypeptide P-G0, G1 and G2 preparation:}

Preparation of P0-a. M-a (1.00 g, $1.72 \mathrm{mmol})$ and DIPEA $(1.78 \mathrm{~mL}, 10.36 \mathrm{mmol})$ were dissolved in $2 \mathrm{~mL}$ of anhydrous DMSO, after which M0 $(0.44 \mathrm{~g}, 1.72 \mathrm{mmol})$ was added. The mixture was stirred under room temperature for $1 \mathrm{~h}$, and then $2 \mathrm{~mL}$ methanol was added to dilute the viscous solution. The polymer was precipitated in diethyl ether, filtered and dried in vacuum for $12 \mathrm{~h}$ to give $0.59 \mathrm{~g}(81 \%) \mathbf{P 0 - a}$.

Preparation of P0-b. P0-a $(0.59 \mathrm{~g}, 1.39 \mathrm{mmol})$ was cooled to $0^{\circ} \mathrm{C}$, after which $2 \mathrm{~mL}$ of TFA was added. The mixture was allowed to stirred under room temperature for $0.5 \mathrm{~h}$. The excess TFA was removed under reduced pressure. The syrup residue was dilute by $3 \mathrm{~mL}$ of methanol and precipitate in diethyl ether. The precipitate was washed by diethyl ether sever time and filtered to give $0.54 \mathrm{~g}(93 \%)$ P0-b.

Preparation of P0-c. M0-b (0.54 g, $1.29 \mathrm{mmol})$ and DIPEA $(0.67 \mathrm{~mL}, 3.88 \mathrm{mmol})$ were dissolved in $1 \mathrm{~mL}$ of anhydrous DMSO, after which M-b $(0.46 \mathrm{~g}, 1.29 \mathrm{mmol})$ was added. The mixture was stirred under room temperature for $1 \mathrm{~h}$, and then $2 \mathrm{~mL}$ methanol was added to dilute the viscous solution. The polymer was precipitated in diethyl ether, filtered and dried in vacuum for $12 \mathrm{~h}$ to give $0.52 \mathrm{~g}(81 \%)$ P0-c.

Preparation of P0. P0-a $(0.52 \mathrm{~g}, 1.04 \mathrm{mmol})$ was cooled to $0^{\circ} \mathrm{C}$, after which $2 \mathrm{~mL}$ of TFA was added. The mixture was allowed to stirred under room temperature for $0.5 \mathrm{~h}$. The excess TFA was removed under reduced pressure. The syrup residue was dilute by $2 \mathrm{~mL}$ of methanol and precipitate in diethyl ether. The precipitate was washed by diethyl ether sever time and filtered to give $0.45 \mathrm{~g}(89 \%)$ P-G0.

P0-a ${ }^{1} \mathrm{H}$ NMR (400 MHz, DMSO) $\delta 9.45$ (s, 1H), 8.85 - 7.47 (m, 2H), $7.39-6.34$ (m, 1H), 4.41 - 4.05 (m, $1 \mathrm{H}), 3.66(\mathrm{dd}, J=56.6,50.3 \mathrm{~Hz}, 3 \mathrm{H}), 3.22-2.88(\mathrm{~m}, 3 \mathrm{H}), 2.32-1.13(\mathrm{~m}, 22 \mathrm{H})$.

P0-b ${ }^{1} \mathrm{H}$ NMR (400 MHz, $\left.\mathrm{D}_{2} \mathrm{O}\right) \delta 4.34-4.04(\mathrm{~m}, 1 \mathrm{H}), 3.88(\mathrm{dd}, J=29.8,17.4 \mathrm{~Hz}, 3 \mathrm{H}), 3.11(\mathrm{~d}, J=26.1 \mathrm{~Hz}$, $2 \mathrm{H}), 2.53(\mathrm{~d}, J=2.4 \mathrm{~Hz}, 1 \mathrm{H}), 2.32(\mathrm{dt}, J=15.3,9.7 \mathrm{~Hz}, 2 \mathrm{H}), 2.04(\mathrm{~s}, 2 \mathrm{H}), 1.69$ (s, 2H), 1.41 (dd, $J=38.0$, $31.6 \mathrm{~Hz}, 4 \mathrm{H})$.

P0-c ${ }^{1} \mathrm{H}$ NMR (400 MHz, DMSO) $\delta 10.31(\mathrm{~s}, 1 \mathrm{H}), 8.92-7.53(\mathrm{~m}, 5 \mathrm{H}), 4.21(\mathrm{dd}, J=26.6,10.3 \mathrm{~Hz}, 4 \mathrm{H}), 3.86$ (d, $J=4.4 \mathrm{~Hz}, 2 \mathrm{H}), 3.23-2.92(\mathrm{~m}, 3 \mathrm{H}), 2.35-1.06(\mathrm{~m}, 21 \mathrm{H})$.

P0 ${ }^{1} \mathrm{H}$ NMR (400 MHz, $\left.\mathrm{D}_{2} \mathrm{O}\right) \delta 4.52-4.01(\mathrm{~m}, 4 \mathrm{H}), 3.99-3.75(\mathrm{~m}, 2 \mathrm{H}), 3.27-2.97(\mathrm{~m}, 2 \mathrm{H}), 2.55(\mathrm{~s}, 1 \mathrm{H})$, $2.30(\mathrm{dd}, J=32.5,6.1 \mathrm{~Hz}, 2 \mathrm{H}), 1.99(\mathrm{dd}, J=37.5,9.3 \mathrm{~Hz}, 2 \mathrm{H}), 1.68(\mathrm{~d}, J=9.4 \mathrm{~Hz}, 2 \mathrm{H}), 1.52-1.15(\mathrm{~m}, 4 \mathrm{H})$. 


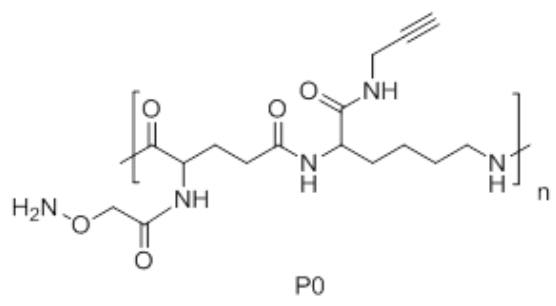

P1-a ${ }^{1} \mathrm{H}$ NMR (400 MHz, DMSO) $\delta 9.07(\mathrm{~s}, 1 \mathrm{H}), 8.65-7.63(\mathrm{~m}, 3 \mathrm{H}), 7.15-6.61(\mathrm{~m}, 1 \mathrm{H}), 4.17(\mathrm{~s}, 1 \mathrm{H}), 3.85$ (s, 2H), $3.61(\mathrm{dd}, J=10.7,6.6 \mathrm{~Hz}, 1 \mathrm{H}), 3.24-2.77(\mathrm{~m}, 4 \mathrm{H}), 2.29-1.04(\mathrm{~m}, 27 \mathrm{H})$.

P1-b ${ }^{1} \mathrm{H}$ NMR (400 MHz, $\left.\mathrm{D}_{2} \mathrm{O}\right) \delta 4.37-4.05(\mathrm{~m}, 2 \mathrm{H}), 4.04-3.75(\mathrm{~m}, 3 \mathrm{H}), 3.08$ (d, $\left.J=6.8 \mathrm{~Hz}, 2 \mathrm{H}\right), 2.99-$ $2.86(\mathrm{~m}, 2 \mathrm{H}), 2.53(\mathrm{~s}, 1 \mathrm{H}), 2.44-2.13(\mathrm{~m}, 2 \mathrm{H}), 1.78(\mathrm{ddd}, J=72.2,24.3,5.8 \mathrm{~Hz}, 8 \mathrm{H}), 1.36(\mathrm{ddd}, J=40.4$, $15.3,7.1 \mathrm{~Hz}, 6 \mathrm{H})$.

P1-c ${ }^{1} \mathrm{H}$ NMR (400 MHz, DMSO) $\delta 10.31$ (s, 1H), 8.16 (ddd, $\left.J=127.6,71.4,48.2 \mathrm{~Hz}, 4 \mathrm{H}\right), 4.46$ - 4.06 (m, $4 \mathrm{H}), 3.85(\mathrm{~s}, 1 \mathrm{H}), 3.60(\mathrm{~s}, 1 \mathrm{H}), 3.35$ (s, 2H), 3.03 (ddd, $J=19.6,15.1,6.7 \mathrm{~Hz}, 5 \mathrm{H}), 2.31-1.06(\mathrm{~m}, 28 \mathrm{H})$. P1 ${ }^{1} \mathrm{H}$ NMR $\left(400 \mathrm{MHz}, \mathrm{D}_{2} \mathrm{O}\right) \delta 4.49-4.02(\mathrm{~m}, 7 \mathrm{H}), 4.00-3.78(\mathrm{~m}, 2 \mathrm{H}), 3.11(\mathrm{dd}, J=23.2,16.8 \mathrm{~Hz}, 4 \mathrm{H})$, $2.54(\mathrm{~s}, 1 \mathrm{H}), 2.27(\mathrm{dd}, J=31.1,6.1 \mathrm{~Hz}, 2 \mathrm{H}), 2.14-1.10(\mathrm{~m}, 15 \mathrm{H})$.

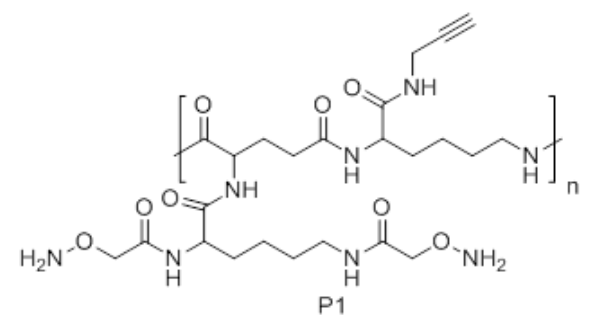

P2-a ${ }^{1} \mathrm{H}$ NMR (400 MHz, DMSO) $\delta 8.62-7.65(\mathrm{~m}, 5 \mathrm{H}), 7.07-6.17(\mathrm{~m}, 3 \mathrm{H}), 4.21(\mathrm{~s}, 2 \mathrm{H}), 4.00-3.49(\mathrm{~m}$, $4 \mathrm{H}), 3.25-2.78(\mathrm{~m}, 8 \mathrm{H}), 2.32-1.01(\mathrm{~m}, 58 \mathrm{H})$

P2-b ${ }^{1} \mathrm{H}$ NMR $\left(400 \mathrm{MHz}, \mathrm{D}_{2} \mathrm{O}\right) \delta 4.30-3.99(\mathrm{~m}, 3 \mathrm{H}), 3.98-3.76(\mathrm{~m}, 4 \mathrm{H}), 3.22-2.97(\mathrm{~m}, 4 \mathrm{H}), 2.89(\mathrm{dd}, J=$ 14.5, $6.9 \mathrm{~Hz}, 4 \mathrm{H}), 2.49$ (d, $J=2.1 \mathrm{~Hz}, 1 \mathrm{H}), 2.22(\mathrm{~d}, J=29.8 \mathrm{~Hz}, 2 \mathrm{H}), 2.08-1.11(\mathrm{~m}, 27 \mathrm{H})$.

P2-c ${ }^{1} \mathrm{H}$ NMR (400 MHz, DMSO) $\delta 10.33$ (s, 2H), $9.54-7.60(\mathrm{~m}, 9 \mathrm{H}), 4.89-4.01(\mathrm{~m}, 8 \mathrm{H}), 3.85$ (s, 2H), 3.57 (s, 6H), 3.09 (d, $J=4.9 \mathrm{~Hz}, 8 \mathrm{H}), 2.28-0.89(\mathrm{~m}, 54 \mathrm{H})$.

P2 ${ }^{1} \mathrm{H}$ NMR $\left(400 \mathrm{MHz}, \mathrm{D}_{2} \mathrm{O}\right) \delta 4.30(\mathrm{dd}, J=63.6,27.3 \mathrm{~Hz}, 1 \mathrm{H}), 4.00-3.76(\mathrm{~m}, 1 \mathrm{H}), 3.13(\mathrm{~d}, J=23.3 \mathrm{~Hz}$, $1 \mathrm{H}), 2.53(\mathrm{~s}, 1 \mathrm{H}), 1.78$ (ddd, $J=156.4,97.4,43.9 \mathrm{~Hz}, 3 \mathrm{H})$.

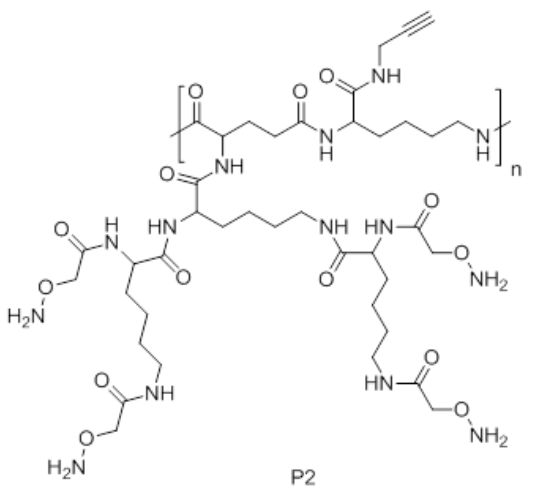


General Procedure for Glycosylation. P0 (20 mg, $0.39 \mathrm{mmol}$ ), tri-Mannose (26 mg, $0.50 \mathrm{mmol}$, acetylation and acetolysis of tri-Mannose from baker's yeast were reported elsewhere $\left.{ }^{1}\right)$ and aniline $(20 \mu \mathrm{L})$ were dissolved in $\mathrm{HAc} / \mathrm{NaAc}$ buffer $\left(\mathrm{pH}=4.2\right.$ ). The solution was allowed to stir under $37^{\circ} \mathrm{C}$ for $12 \mathrm{~h}$, and dialysis against distilled water. The resulted solution was lyophilized to give $40 \mathrm{mg}(95 \%)$ P0tM

tri-Mannose (OAc): $\quad{ }^{1} \mathrm{H}$ NMR $\left(400 \mathrm{MHz}, \mathrm{CDCl}_{3}\right) \delta 6.26(\mathrm{~d}, J=2.2 \mathrm{~Hz}, 1 \mathrm{H}), 5.58-5.20(\mathrm{~m}, 7 \mathrm{H}), 5.14(\mathrm{~d}, J$ $=2.1 \mathrm{~Hz}, 1 \mathrm{H}), 4.96(\mathrm{~d}, J=1.8 \mathrm{~Hz}, 1 \mathrm{H}), 4.39-3.93(\mathrm{~m}, 11 \mathrm{H}), 2.38-1.83(\mathrm{~m}, 38 \mathrm{H})$.

${ }^{13} \mathrm{C}$ NMR $\left(101 \mathrm{MHz}, \mathrm{CDCl}_{3}\right) \delta 170.89,170.47,170.20,170.04,169.77,169.47,169.36,169.26,168.26,99.88$, $99.38,91.55,75.45,70.78,69.98,69.67,69.55,69.51,68.40,66.26,66.05,65.54,62.48,62.19,61.67,20.89$, $20.84,20.71,20.69,20.67,20.63,20.61,20.57$

tri-Mannose: ${ }^{1} \mathrm{H}$ NMR $\left(400 \mathrm{MHz}, \mathrm{CDCl}_{3}\right) \delta 6.26(\mathrm{~d}, J=2.2 \mathrm{~Hz}, 1 \mathrm{H}), 5.58-5.20(\mathrm{~m}, 7 \mathrm{H}), 5.14(\mathrm{~d}, J=2.1 \mathrm{~Hz}$, $1 \mathrm{H}), 4.96(\mathrm{~d}, J=1.8 \mathrm{~Hz}, 1 \mathrm{H}), 4.39-3.93(\mathrm{~m}, 11 \mathrm{H}), 2.38-1.83(\mathrm{~m}, 38 \mathrm{H})$.

${ }^{13} \mathrm{C}$ NMR $\left(101 \mathrm{MHz}, \mathrm{D}_{2} \mathrm{O}\right) \delta 102.21,100.53,92.46,79.31,78.52,73.20,72.43,70.31,69.96,69.92,67.01$, $66.80,61.03,60.92$
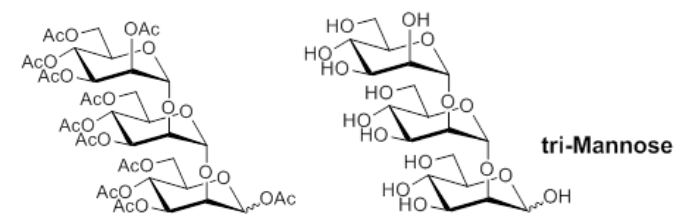

P0M ${ }^{1} \mathrm{H}$ NMR $\left(400 \mathrm{MHz}, \mathrm{D}_{2} \mathrm{O}\right) \delta 7.77-7.50(\mathrm{~m}, 1 \mathrm{H}), 7.08-6.86(\mathrm{~m}, 1 \mathrm{H}), 4.93(\mathrm{t}, J=7.6 \mathrm{~Hz}, 1 \mathrm{H}), 4.52(\mathrm{~s}$, 1H), 4.19 (dd, $J=19.4,11.3 \mathrm{~Hz}, 3 \mathrm{H}), 4.00-3.45(\mathrm{~m}, 6 \mathrm{H}), 3.07$ (d, $J=6.4 \mathrm{~Hz}, 2 \mathrm{H}), 2.50(\mathrm{~s}, 1 \mathrm{H}), 2.25$ (dd, $J=$ 39.3, 7.8 Hz, 2H), 1.94 (ddd, $J=21.2,14.8,7.8 \mathrm{~Hz}, 2 \mathrm{H}), 1.65$ (s, 2H), 1.33 (d, $J=60.4 \mathrm{~Hz}, 4 \mathrm{H}$ ).

POdM ${ }^{1} \mathrm{H}$ NMR (400 MHz, $\left.\mathrm{D}_{2} \mathrm{O}\right) \delta 7.80-7.53(\mathrm{~m}, 1 \mathrm{H}), 7.03(\mathrm{~d}, J=7.0 \mathrm{~Hz}, 1 \mathrm{H}), 5.01(\mathrm{t}, J=7.8 \mathrm{~Hz}, 1 \mathrm{H})$, $4.87(\mathrm{~s}, 1 \mathrm{H}), 4.53(\mathrm{~s}, 1 \mathrm{H}), 4.36-4.05(\mathrm{~m}, 3 \mathrm{H}), 4.03-3.46(\mathrm{~m}, 12 \mathrm{H}), 3.07(\mathrm{~d}, J=6.6 \mathrm{~Hz}, 2 \mathrm{H}), 2.51(\mathrm{~s}, 1 \mathrm{H})$, $2.38-2.10(\mathrm{~m}, 2 \mathrm{H}), 2.09-1.79(\mathrm{~m}, 2 \mathrm{H}), 1.65(\mathrm{~s}, 2 \mathrm{H}), 1.40(\mathrm{~s}, 2 \mathrm{H}), 1.25(\mathrm{~s}, 2 \mathrm{H})$.

P0tM ${ }^{1} \mathrm{H}$ NMR (400 MHz, $\left.\mathrm{D}_{2} \mathrm{O}\right) \delta 7.64(\mathrm{~d}, J=7.5 \mathrm{~Hz}, 1 \mathrm{H}), 7.02(\mathrm{~s}, 1 \mathrm{H}), 5.14(\mathrm{~d}, J=10.1 \mathrm{~Hz}, 1 \mathrm{H}), 4.96(\mathrm{~d}, J$ $=33.0 \mathrm{~Hz}, 1 \mathrm{H}), 4.53(\mathrm{~s}, 1 \mathrm{H}), 4.13(\mathrm{dd}, J=16.5,8.1 \mathrm{~Hz}, 3 \mathrm{H}), 4.03-3.35(\mathrm{~m}, 14 \mathrm{H}), 3.08(\mathrm{~s}, 2 \mathrm{H}), 2.51(\mathrm{~s}, 1 \mathrm{H})$, $2.24(\mathrm{~d}, J=32.5 \mathrm{~Hz}, 2 \mathrm{H}), 2.10-1.78(\mathrm{~m}, 3 \mathrm{H}), 1.65(\mathrm{~s}, 2 \mathrm{H}), 1.33(\mathrm{~d}, J=60.4 \mathrm{~Hz}, 4 \mathrm{H})$.

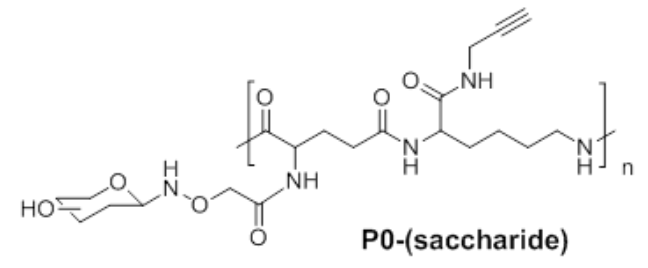

P1M ${ }^{1} \mathrm{H}$ NMR $\left(400 \mathrm{MHz}, \mathrm{D}_{2} \mathrm{O}\right) \delta 7.92(\mathrm{~s}, 1 \mathrm{H}), 7.64(\mathrm{t}, J=7.1 \mathrm{~Hz}, 1 \mathrm{H}), 7.06-6.89(\mathrm{~m}, 1 \mathrm{H}), 4.91(\mathrm{t}, J=7.6$ $\mathrm{Hz}, 1 \mathrm{H}), 4.61-4.40(\mathrm{~m}, 2 \mathrm{H}), 4.18(\mathrm{ddd}, J=34.9,19.8,15.2 \mathrm{~Hz}, 5 \mathrm{H}), 3.99-3.41(\mathrm{~m}, 11 \mathrm{H}), 3.10(\mathrm{~d}, J=28.5$ $\mathrm{Hz}, 4 \mathrm{H}), 2.51(\mathrm{~s}, 1 \mathrm{H}), 2.25(\mathrm{~d}, J=31.0 \mathrm{~Hz}, 2 \mathrm{H}), 2.07-1.06(\mathrm{~m}, 15 \mathrm{H})$

P1dM ${ }^{1} \mathrm{H}$ NMR $\left(400 \mathrm{MHz}, \mathrm{D}_{2} \mathrm{O}\right) \delta 7.64(\mathrm{~d}, J=7.9 \mathrm{~Hz}, 1 \mathrm{H}), 7.01(\mathrm{~d}, J=7.4 \mathrm{~Hz}, 1 \mathrm{H}), 5.00(\mathrm{t}, J=7.8 \mathrm{~Hz}, 1 \mathrm{H})$, $4.87(\mathrm{~d}, J=5.2 \mathrm{~Hz}, 1 \mathrm{H}), 4.51(\mathrm{~d}, J=28.9 \mathrm{~Hz}, 2 \mathrm{H}), 4.16(\mathrm{dd}, J=25.9,17.7 \mathrm{~Hz}, 5 \mathrm{H}), 4.02-3.47(\mathrm{~m}, 21 \mathrm{H})$, $3.10(\mathrm{~d}, J=25.2 \mathrm{~Hz}, 4 \mathrm{H}), 2.51(\mathrm{~s}, 1 \mathrm{H}), 2.24(\mathrm{~d}, J=29.1 \mathrm{~Hz}, 2 \mathrm{H}), 1.85(\mathrm{dd}, J=72.6,48.3 \mathrm{~Hz}, 6 \mathrm{H}), 1.33(\mathrm{~d}, J$ $=72.9 \mathrm{~Hz}, 8 \mathrm{H})$. 
P1tM ${ }^{1} \mathrm{H}$ NMR (400 MHz, $\left.\mathrm{D}_{2} \mathrm{O}\right) \delta 7.66(\mathrm{~d}, J=7.8 \mathrm{~Hz}, 1 \mathrm{H}), 7.03(\mathrm{~d}, J=7.5 \mathrm{~Hz}, 1 \mathrm{H}), 5.42-4.81(\mathrm{~m}, 3 \mathrm{H})$, $4.64-4.06(\mathrm{~m}, 6 \mathrm{H}), 4.04-3.39(\mathrm{~m}, 27 \mathrm{H}), 3.13(\mathrm{~d}, J=30.1 \mathrm{~Hz}, 4 \mathrm{H}), 2.55(\mathrm{~s}, 1 \mathrm{H}), 2.27(\mathrm{~d}, J=29.7 \mathrm{~Hz}, 2 \mathrm{H})$, $2.12-1.08(\mathrm{~m}, 15 \mathrm{H})$.

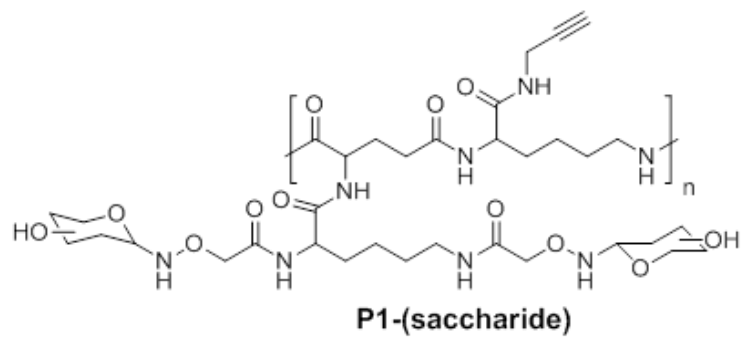

P2M ${ }^{1} \mathrm{H}$ NMR $\left(400 \mathrm{MHz}, \mathrm{D}_{2} \mathrm{O}\right) \delta 7.80-7.56(\mathrm{~m}, 3 \mathrm{H}), 7.08-6.89(\mathrm{~m}, 1 \mathrm{H}), 5.05-4.84(\mathrm{~m}, 1 \mathrm{H}), 4.51(\mathrm{~d}, J=$ $26.0 \mathrm{~Hz}, 4 \mathrm{H}), 4.38-4.05(\mathrm{~m}, 9 \mathrm{H}), 4.01-3.46(\mathrm{~m}, 19 \mathrm{H}), 3.12(\mathrm{~d}, J=19.2 \mathrm{~Hz}, 8 \mathrm{H}), 2.54(\mathrm{~s}, 1 \mathrm{H}), 2.25(\mathrm{~d}, J=$ $27.9 \mathrm{~Hz}, 2 \mathrm{H}), 2.10-1.06(\mathrm{~m}, 28 \mathrm{H})$.

P2dM ${ }^{1} \mathrm{H}$ NMR $\left(400 \mathrm{MHz}, \mathrm{D}_{2} \mathrm{O}\right) \delta 7.63(\mathrm{~d}, J=7.9 \mathrm{~Hz}, 1 \mathrm{H}), 7.00(\mathrm{~d}, J=7.1 \mathrm{~Hz}, 1 \mathrm{H}), 5.00(\mathrm{t}, J=7.8 \mathrm{~Hz}, 1 \mathrm{H})$, $4.86(\mathrm{~d}, J=3.6 \mathrm{~Hz}, 1 \mathrm{H}), 4.61-4.04(\mathrm{~m}, 2 \mathrm{H}), 4.03-3.46(\mathrm{~m}, 5 \mathrm{H}), 3.11(\mathrm{~d}, J=13.7 \mathrm{~Hz}, 1 \mathrm{H}), 2.53(\mathrm{~s}, 1 \mathrm{H})$, $2.40-0.98(\mathrm{~m}, 4 \mathrm{H})$.

P2tM ${ }^{1} \mathrm{H}$ NMR (400 MHz, $\left.\mathrm{D}_{2} \mathrm{O}\right) \delta 7.65(\mathrm{~d}, J=7.8 \mathrm{~Hz}, 3 \mathrm{H}), 7.02(\mathrm{~d}, J=7.0 \mathrm{~Hz}, 1 \mathrm{H}), 5.27-4.82(\mathrm{~m}, 5 \mathrm{H})$, $4.68-4.07(\mathrm{~m}, 12 \mathrm{H}), 4.06-3.40(\mathrm{~m}, 48 \mathrm{H}), 3.15(\mathrm{~s}, 8 \mathrm{H}), 2.55$ (s, 1H), 2.26 (d, $J=34.8 \mathrm{~Hz}, 2 \mathrm{H}), 1.74$ (ddd, $J$ $=214.2,122.7,57.6 \mathrm{~Hz}, 29 \mathrm{H})$.

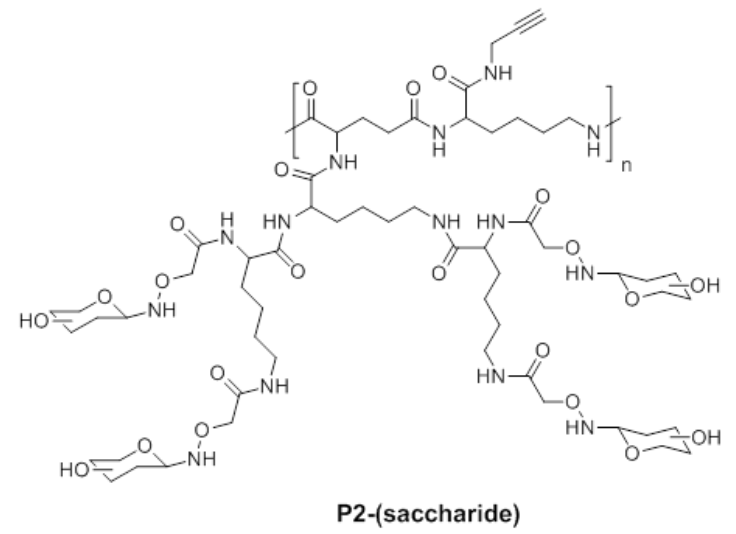

General Procedure for click reaction and self-assembly. P1tM (20 mg, $13 \mu \mathrm{mol}$, monomer unit), M-d (10 $\mathrm{mg}, 13 \mu \mathrm{mol})$ and PMEDTA $(2 \mu \mathrm{L}, 10 \mu \mathrm{mol})$ was dissolved in $1 \mathrm{~mL}$ DMSO with 5\% LiBr. CuBr $(1.5 \mathrm{mg}, 10$ $\mu \mathrm{mol}$ ) was added under $\mathrm{N}_{2}$ atmosphere, and the mixture was allowed to stir under $50^{\circ} \mathrm{C}$ for $12 \mathrm{~h}$. The resulted DMSO solution was diluted with $2 \mathrm{~mL} \mathrm{H}_{2} \mathrm{O}$, and dialysis against distilled water to trigger the self-assembly. Opalescence appears in the solution after dialysis overnight, the samples were characterized without further purification. 


\section{X. ${ }^{1} \mathrm{H}$ and ${ }^{13} \mathrm{C}$ NMR spectra}

1.
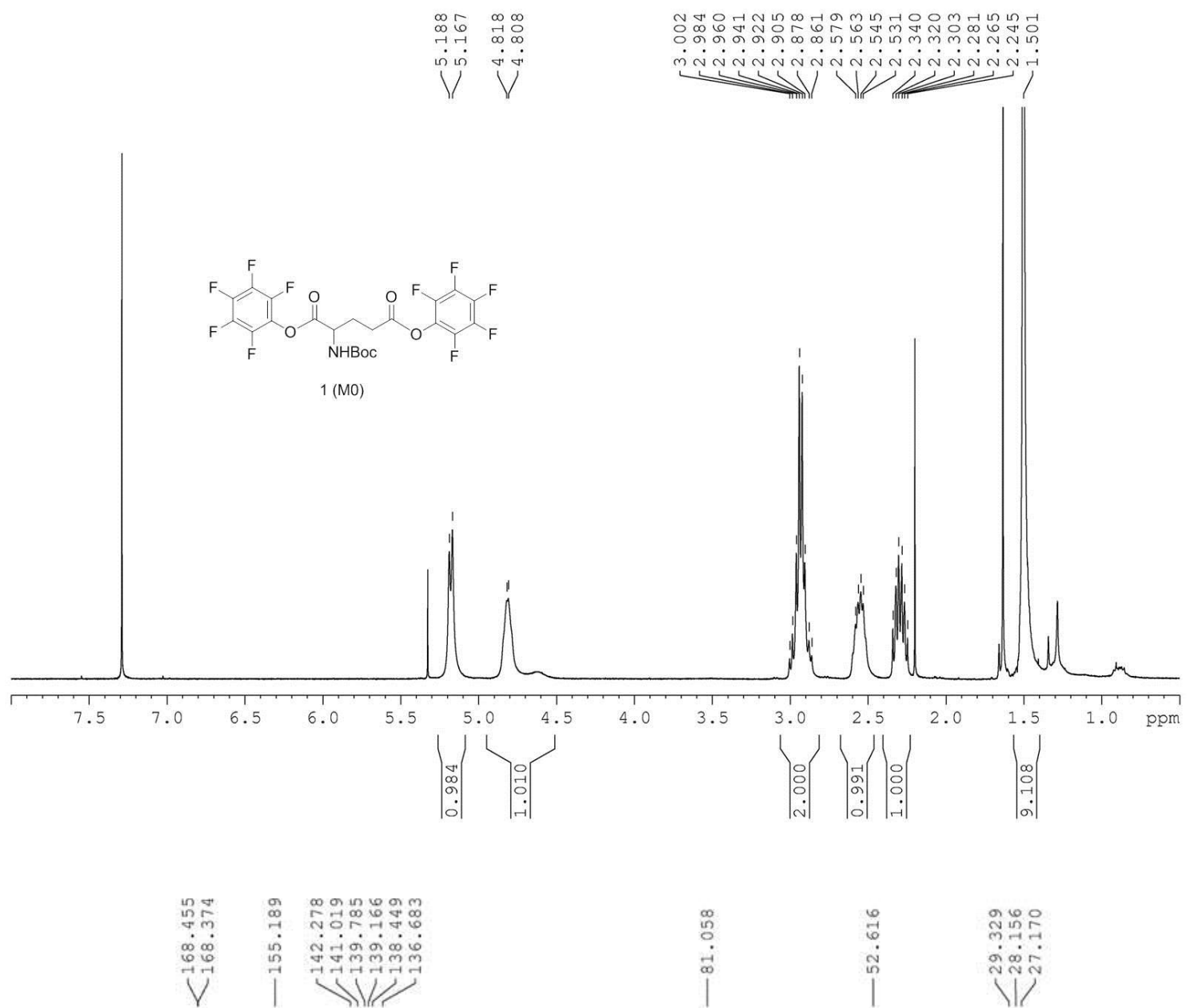

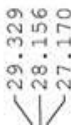
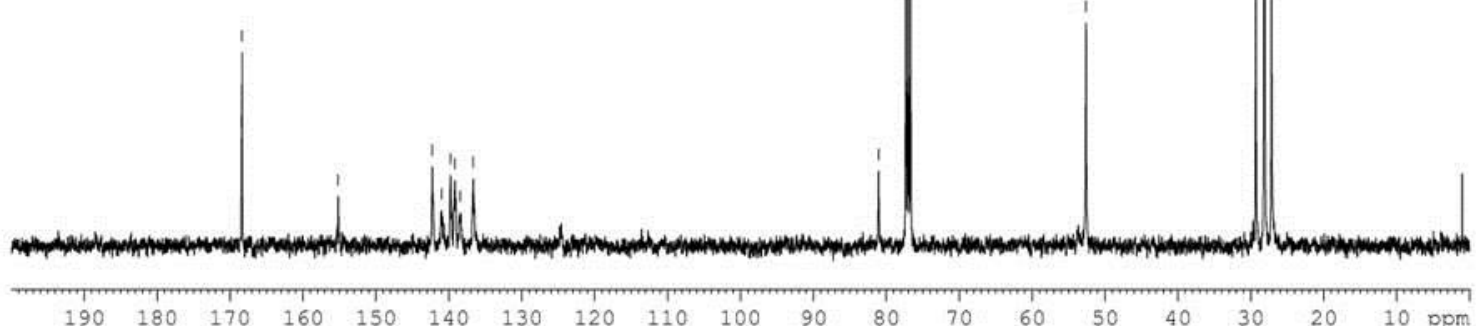
2.
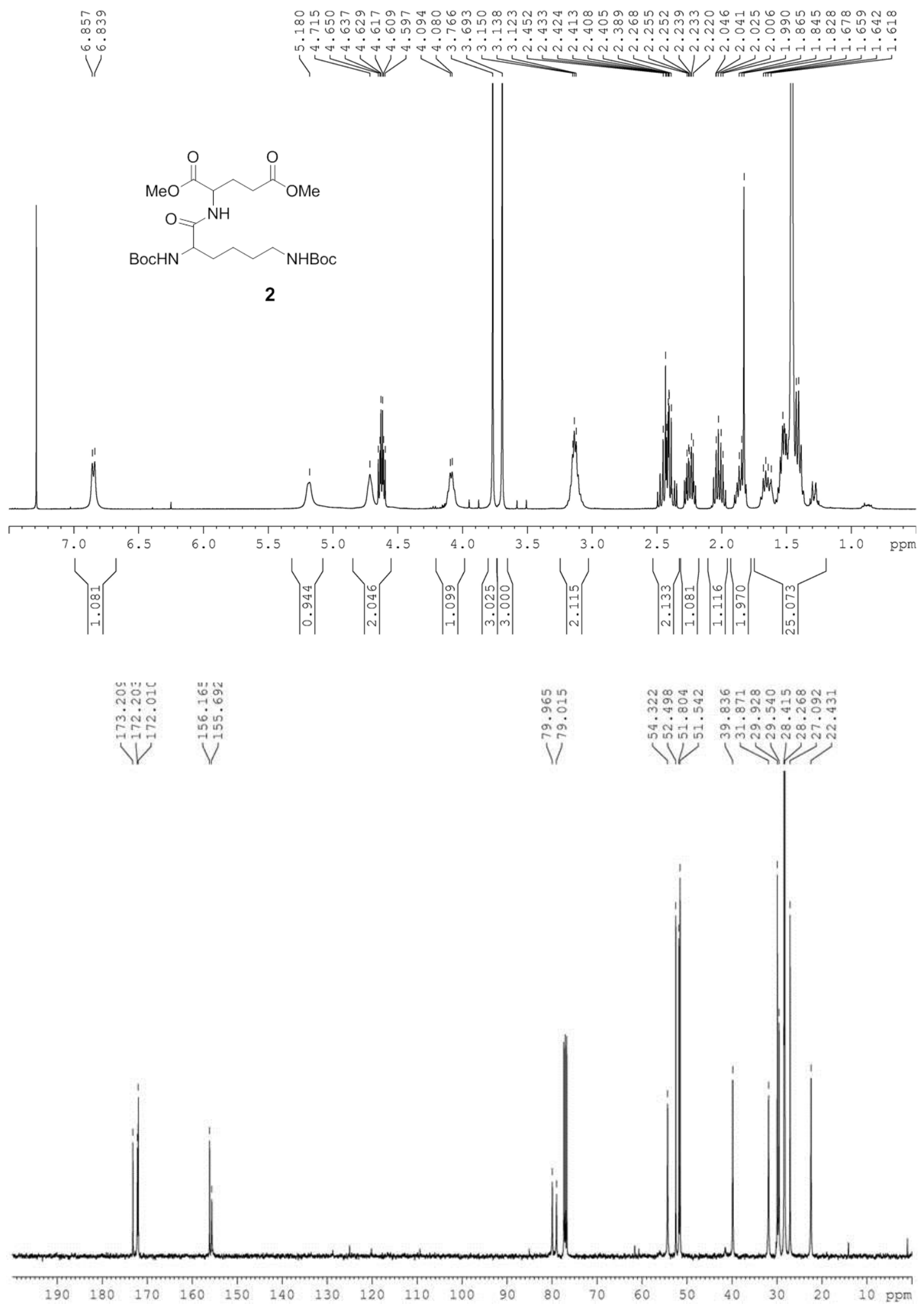
3.
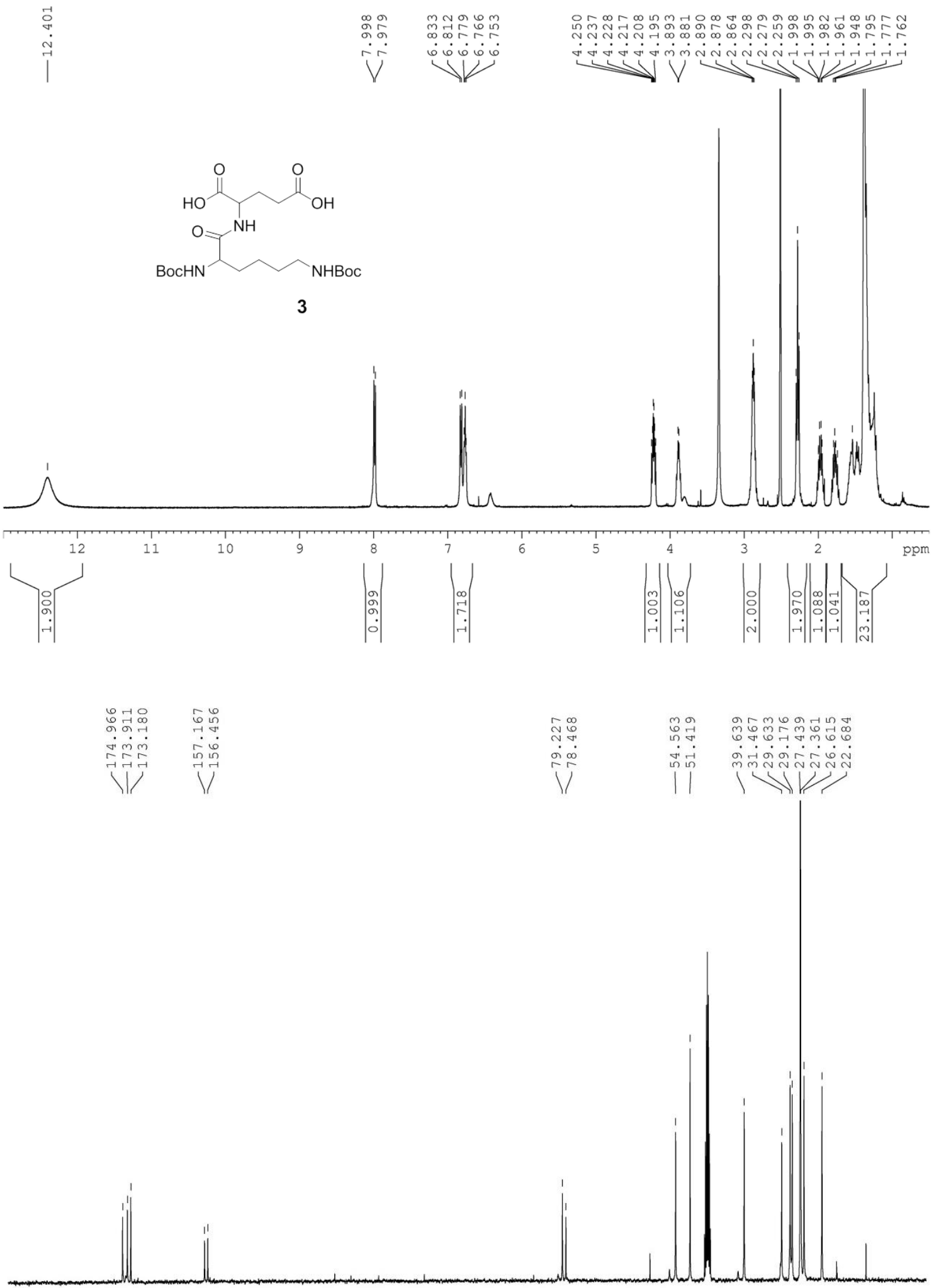

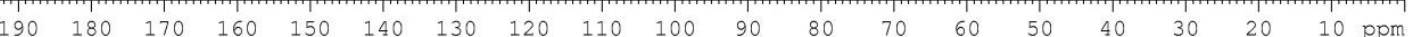


4.

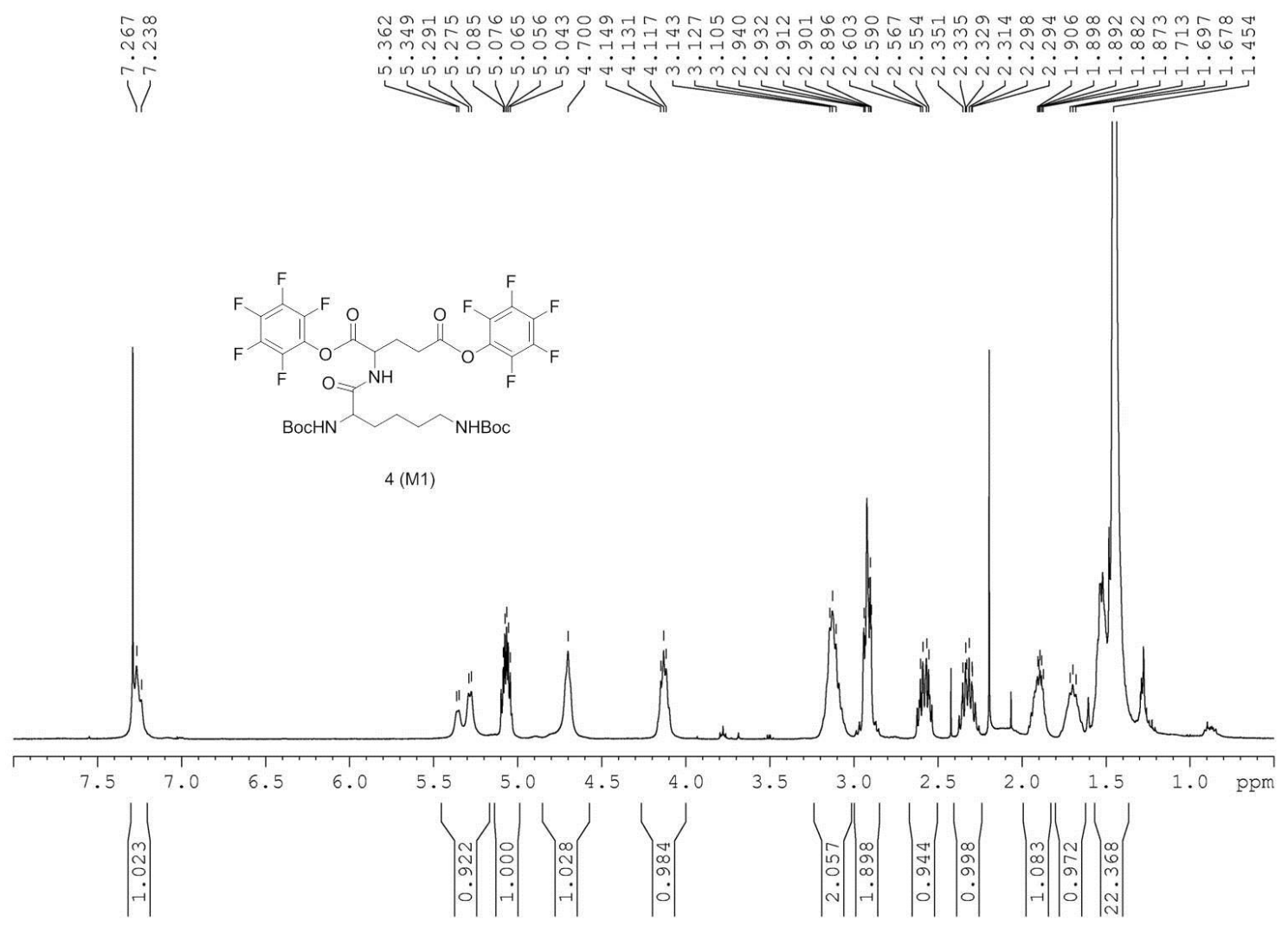

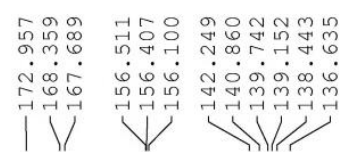
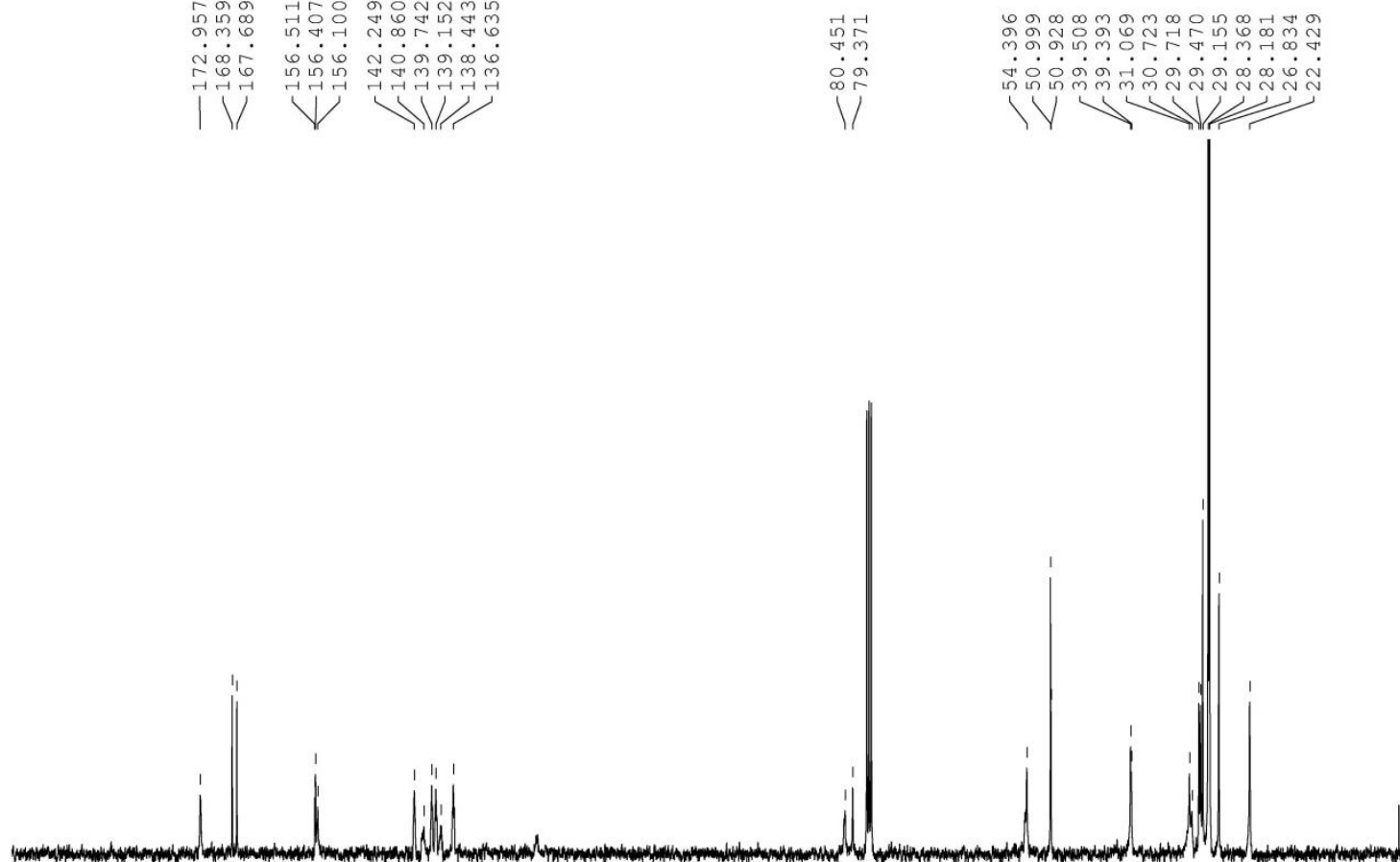

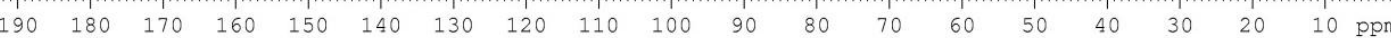


5.

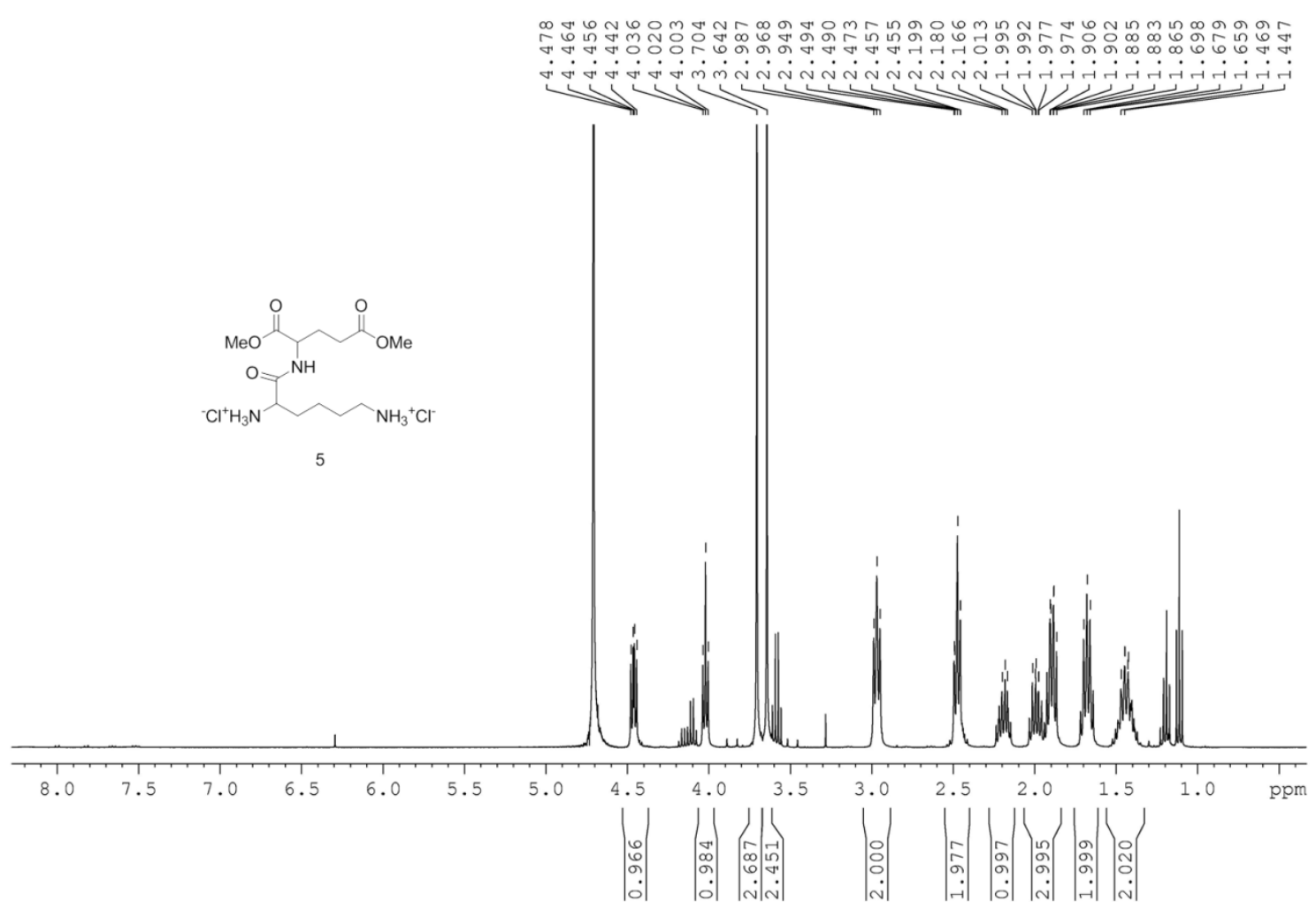

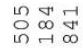

ค่

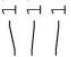
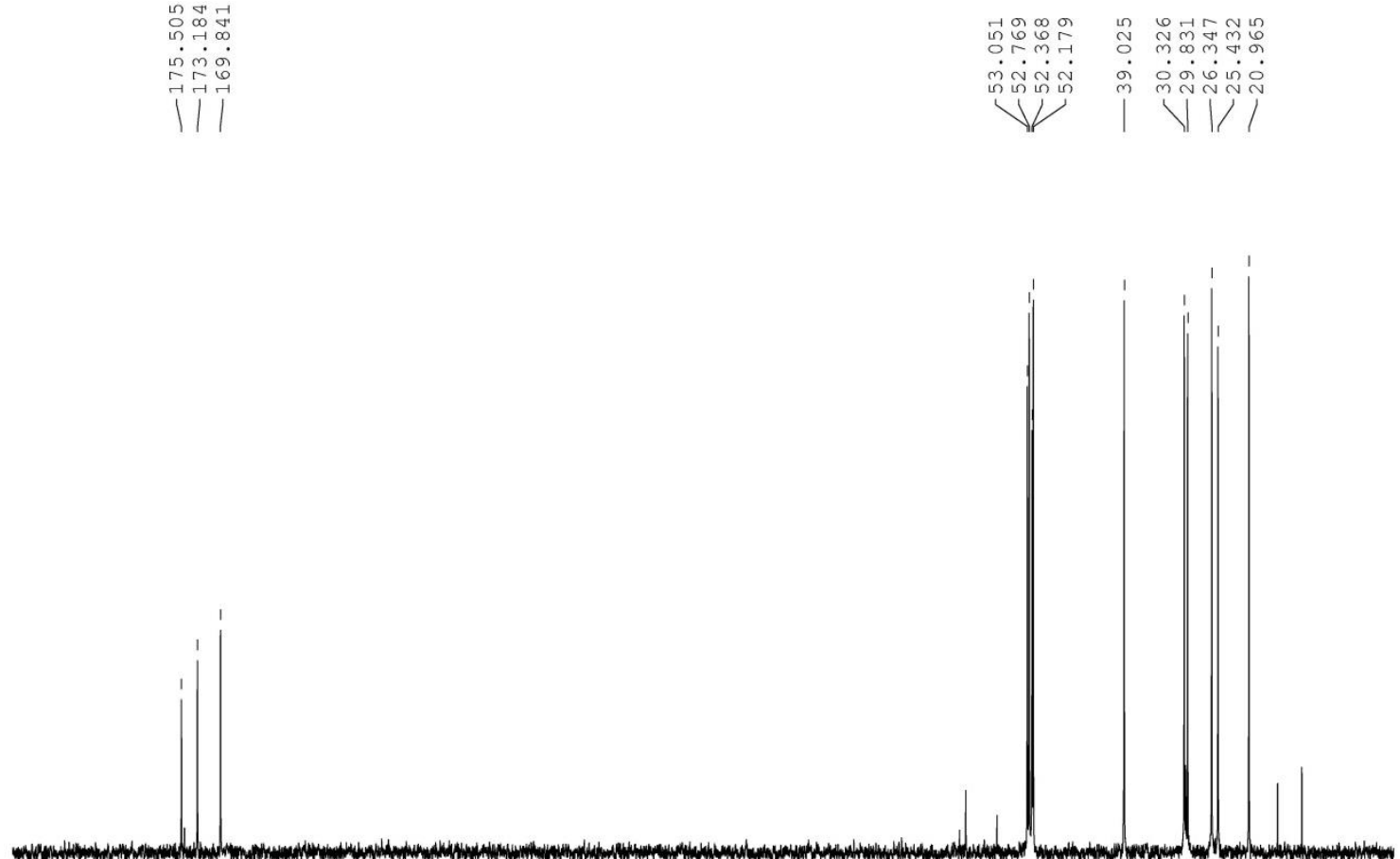

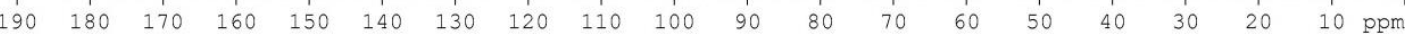


6.

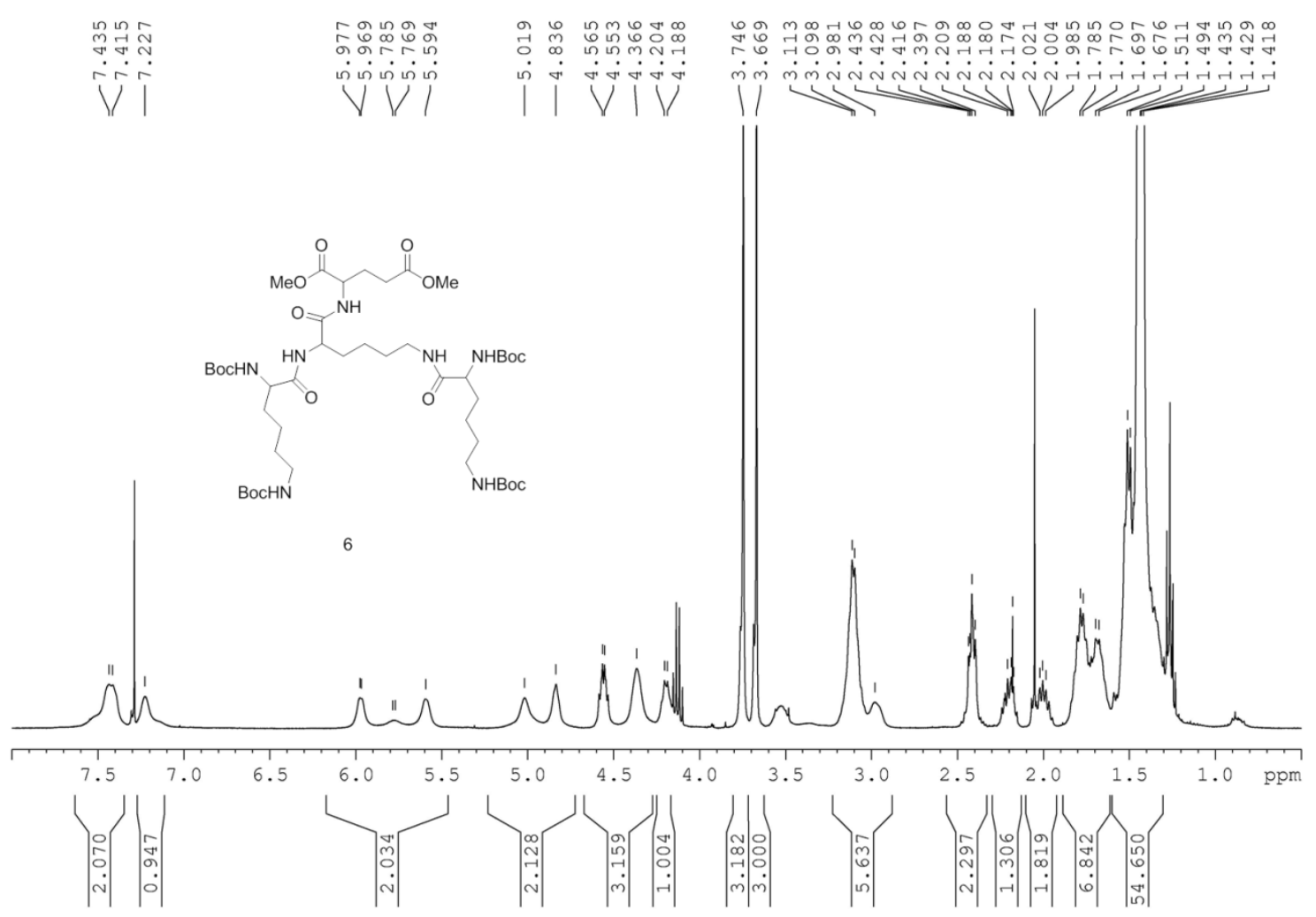

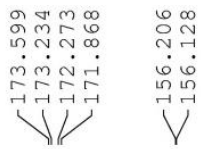
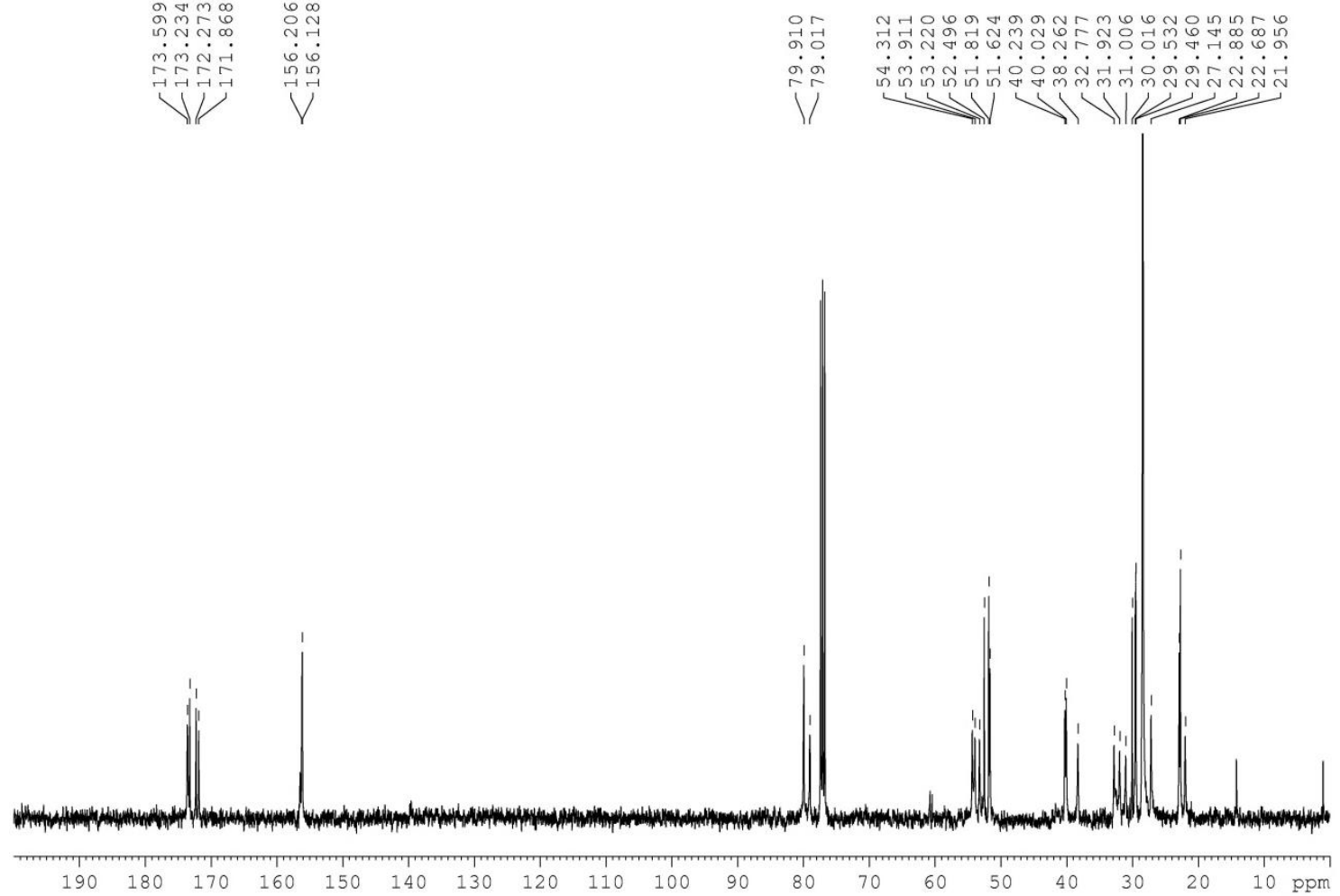
7.
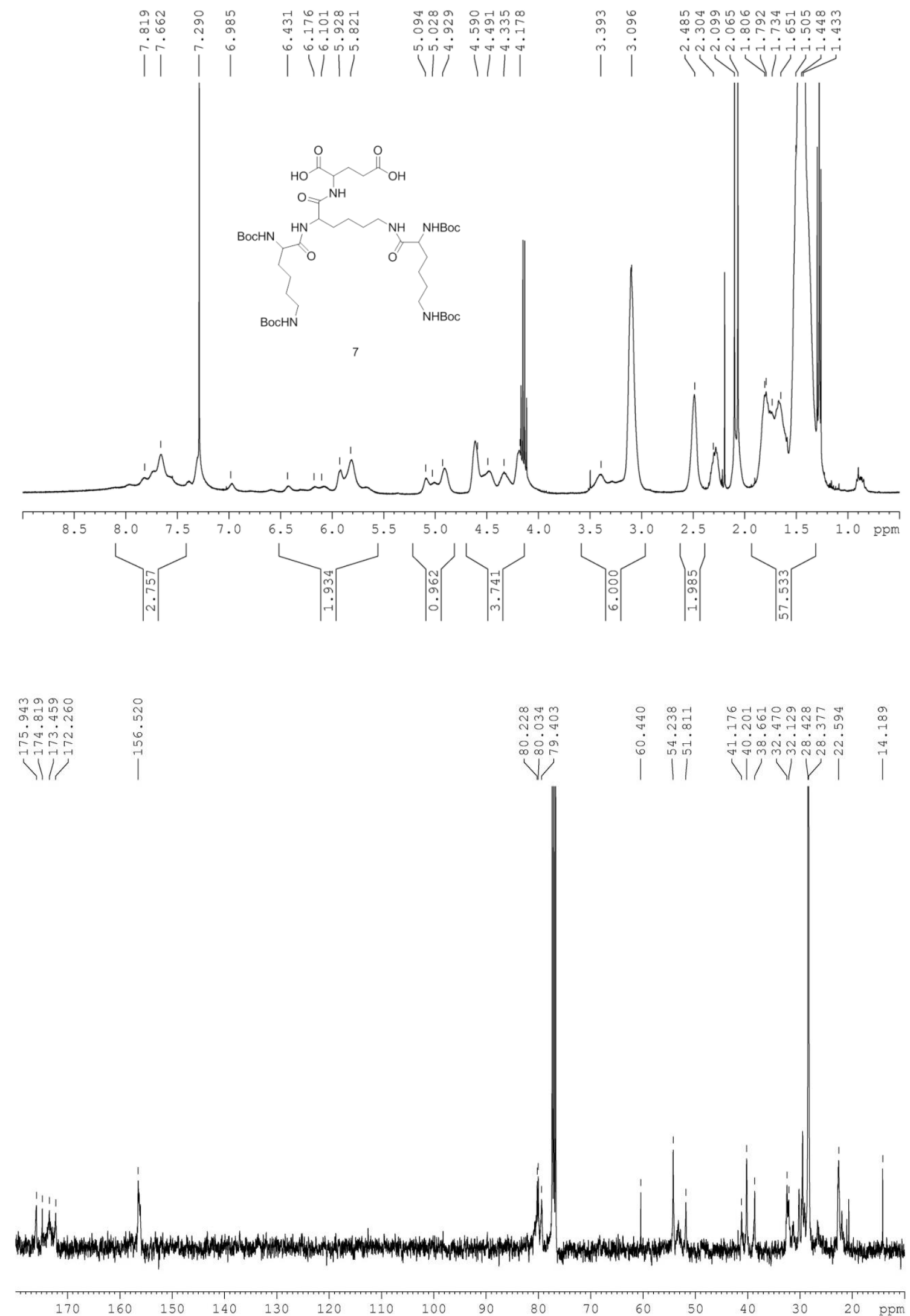
8.
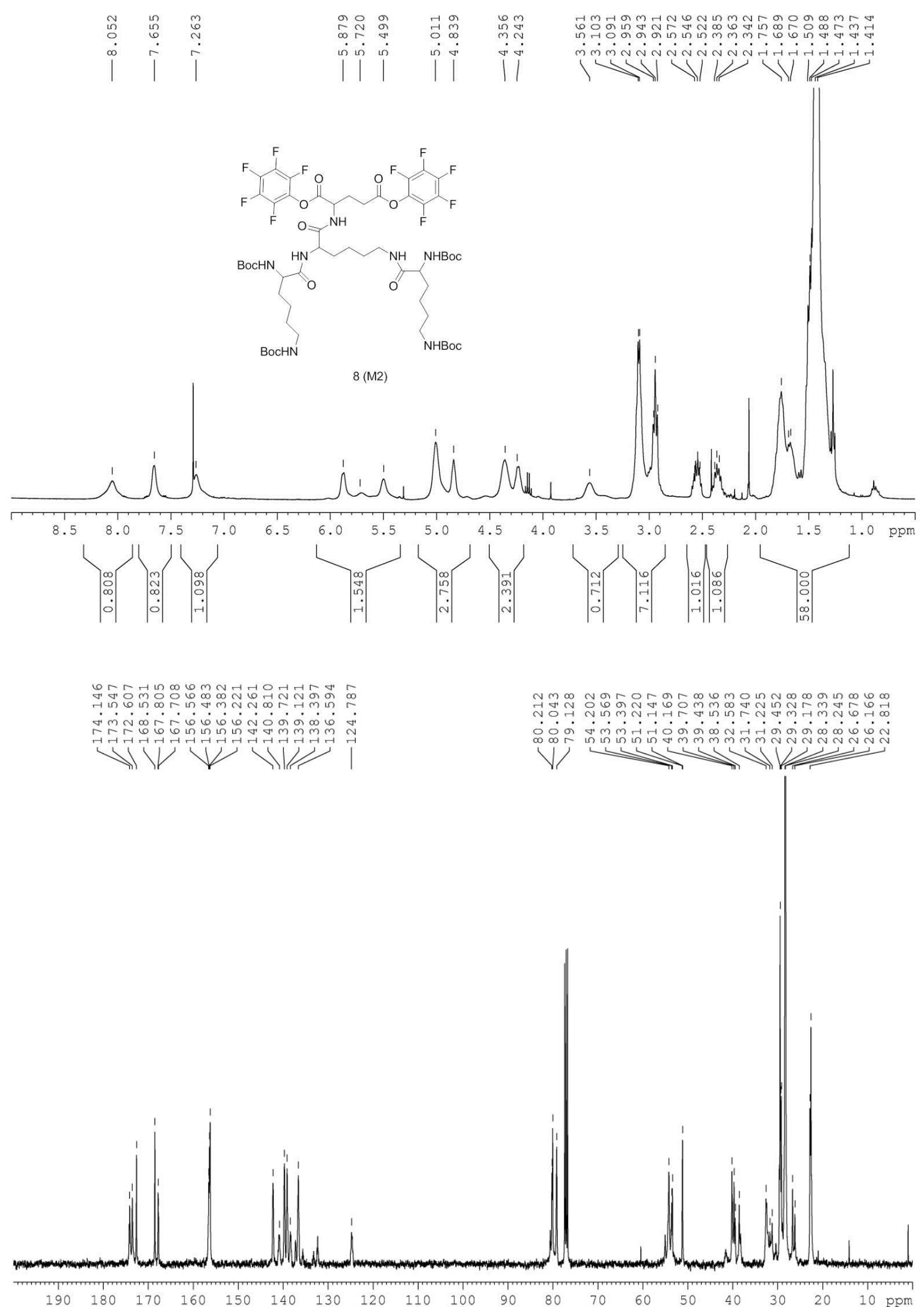
9.
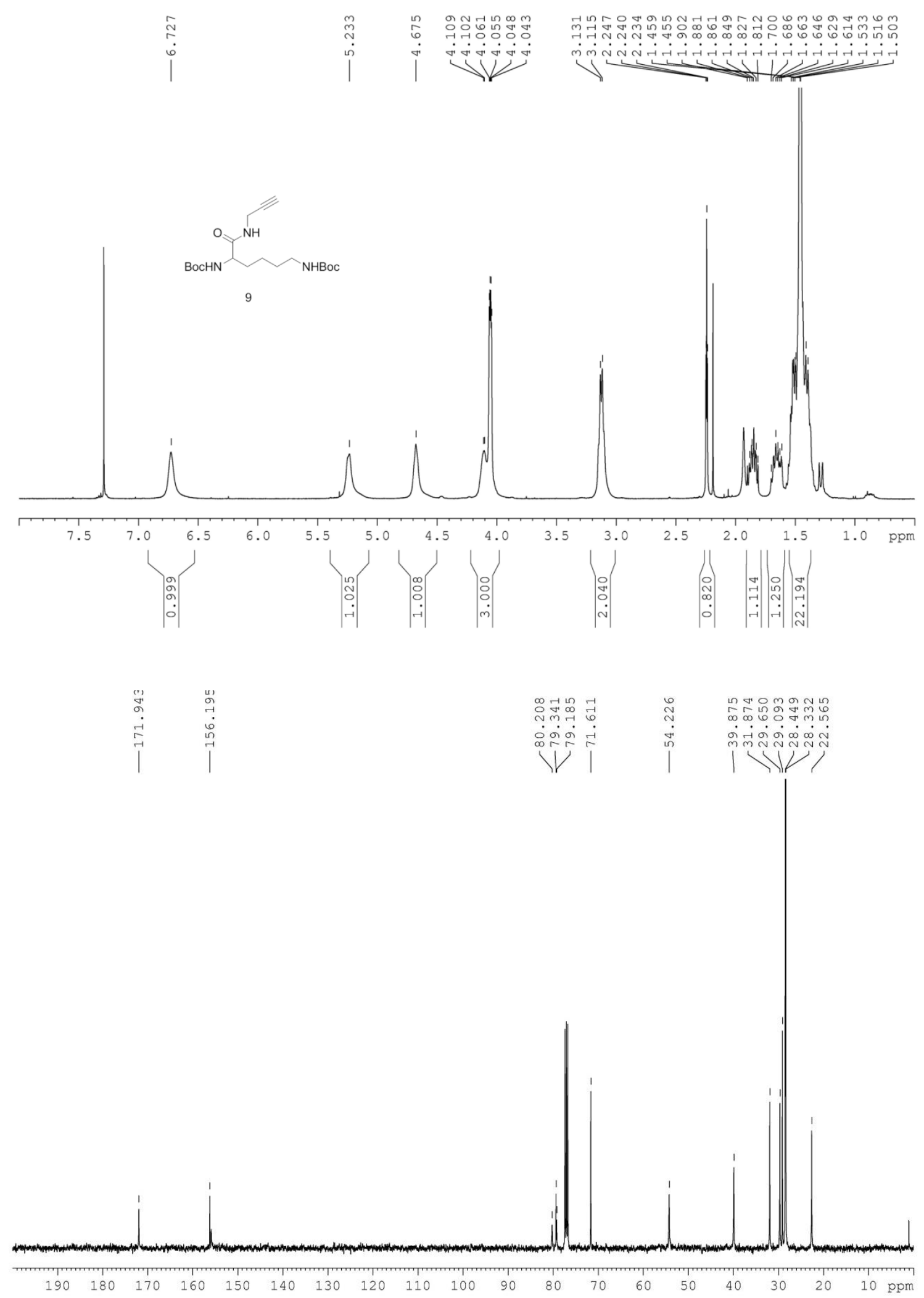
10.
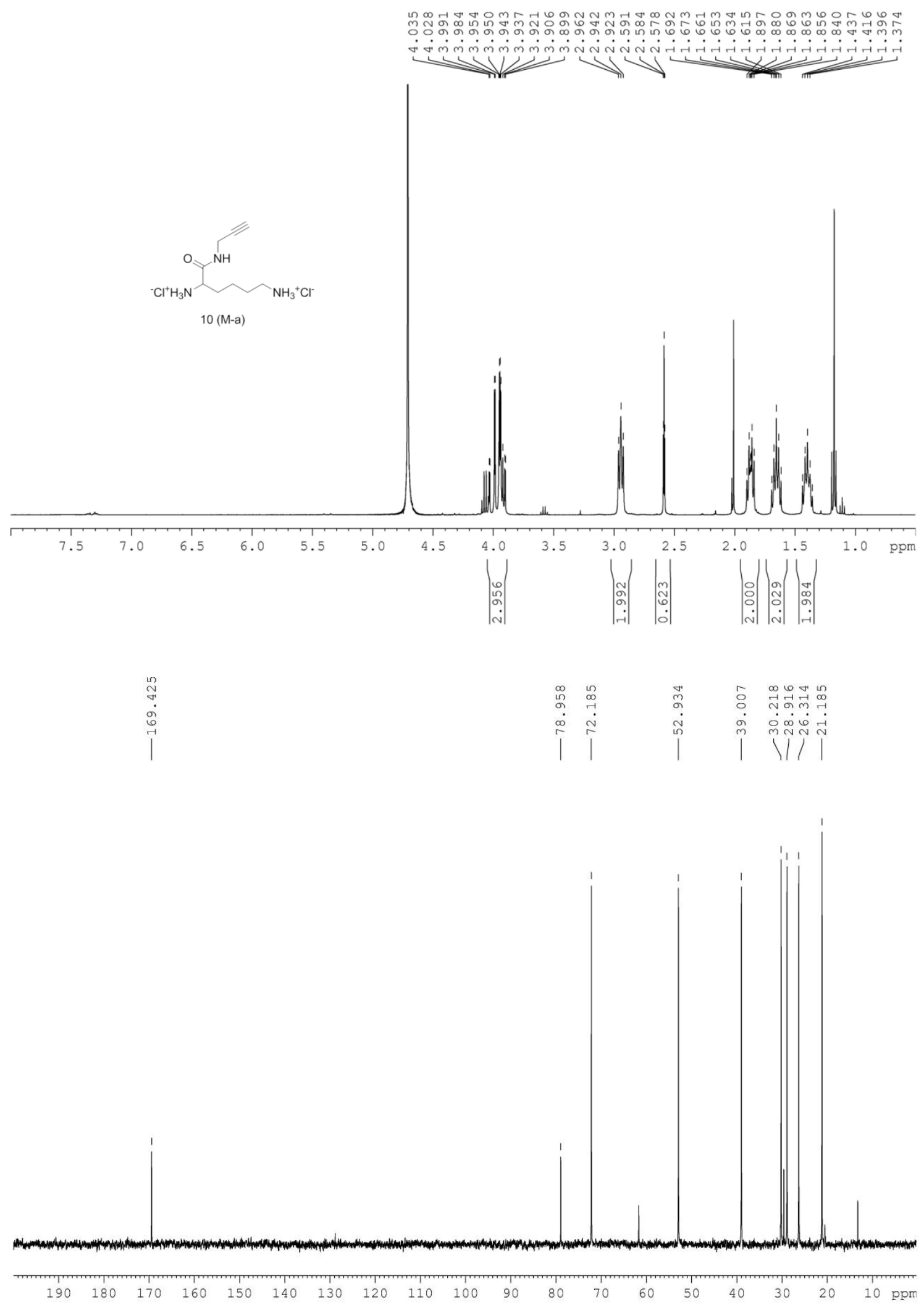
11.
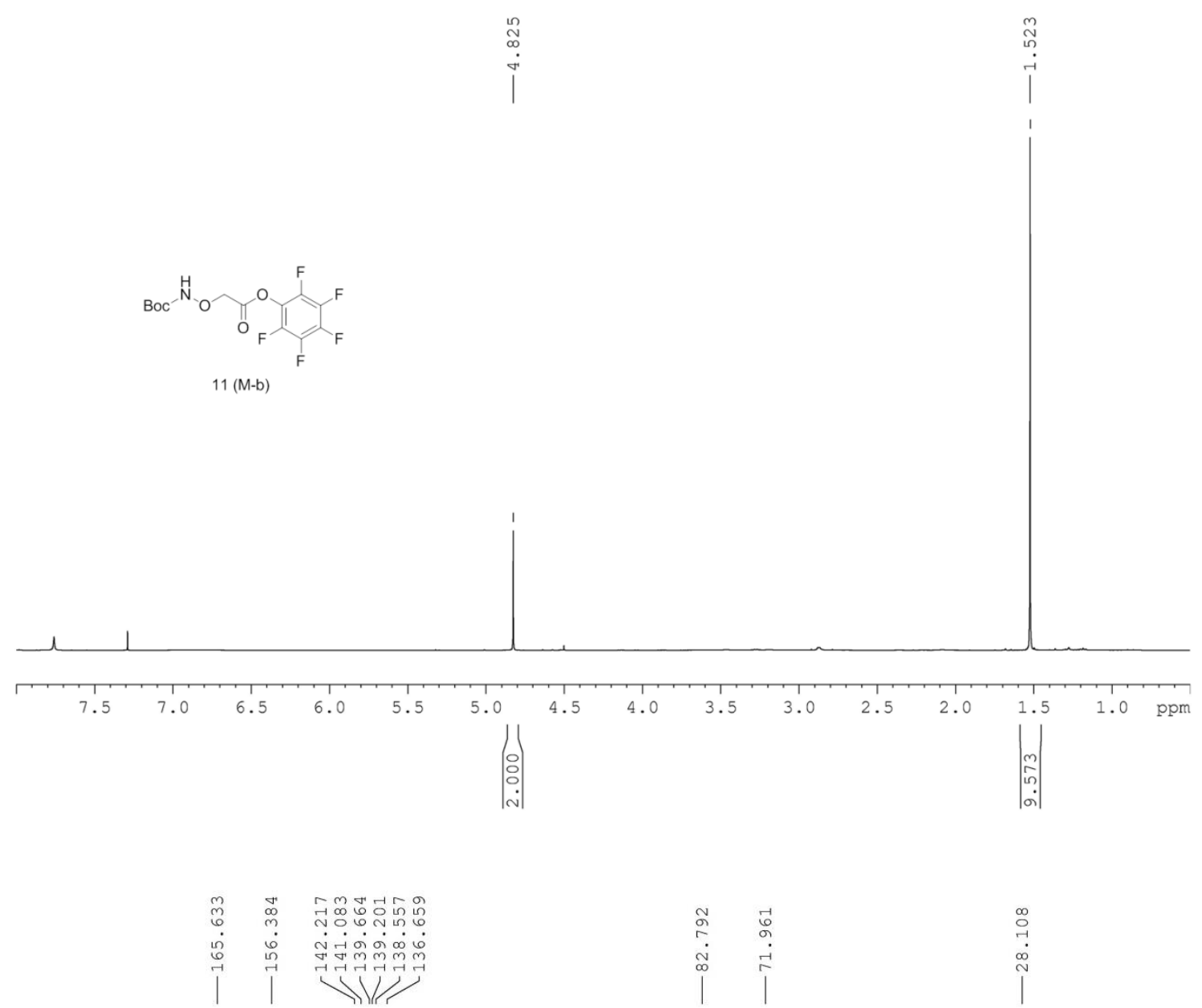

$\infty$
0
+1
$\infty$
$\sim$
$\sim$

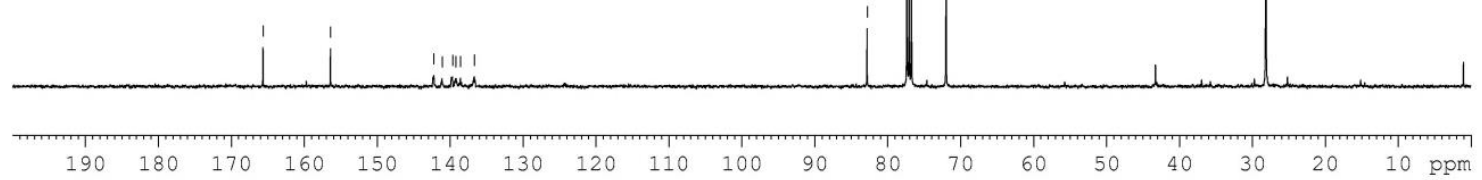


12.
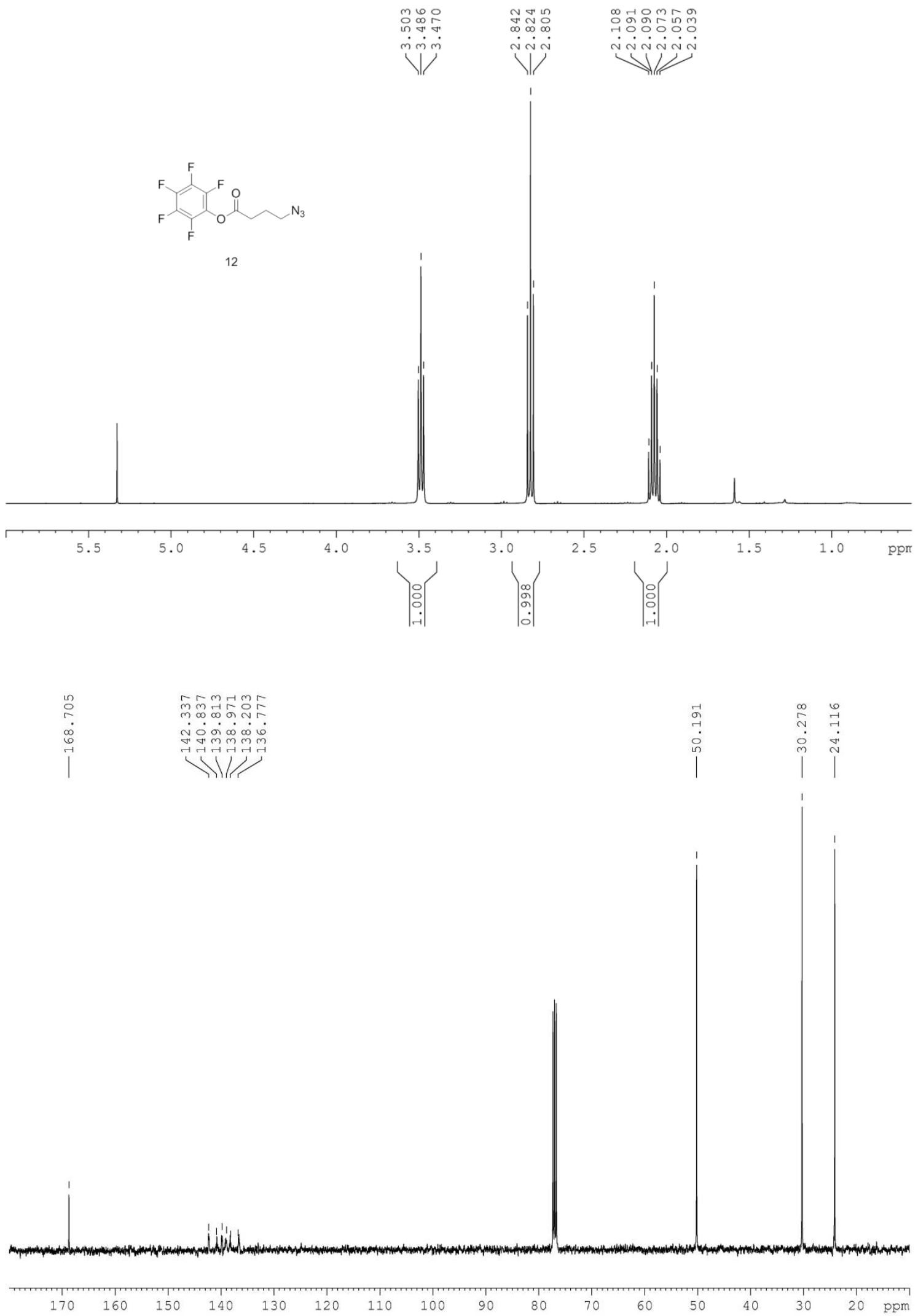
13.
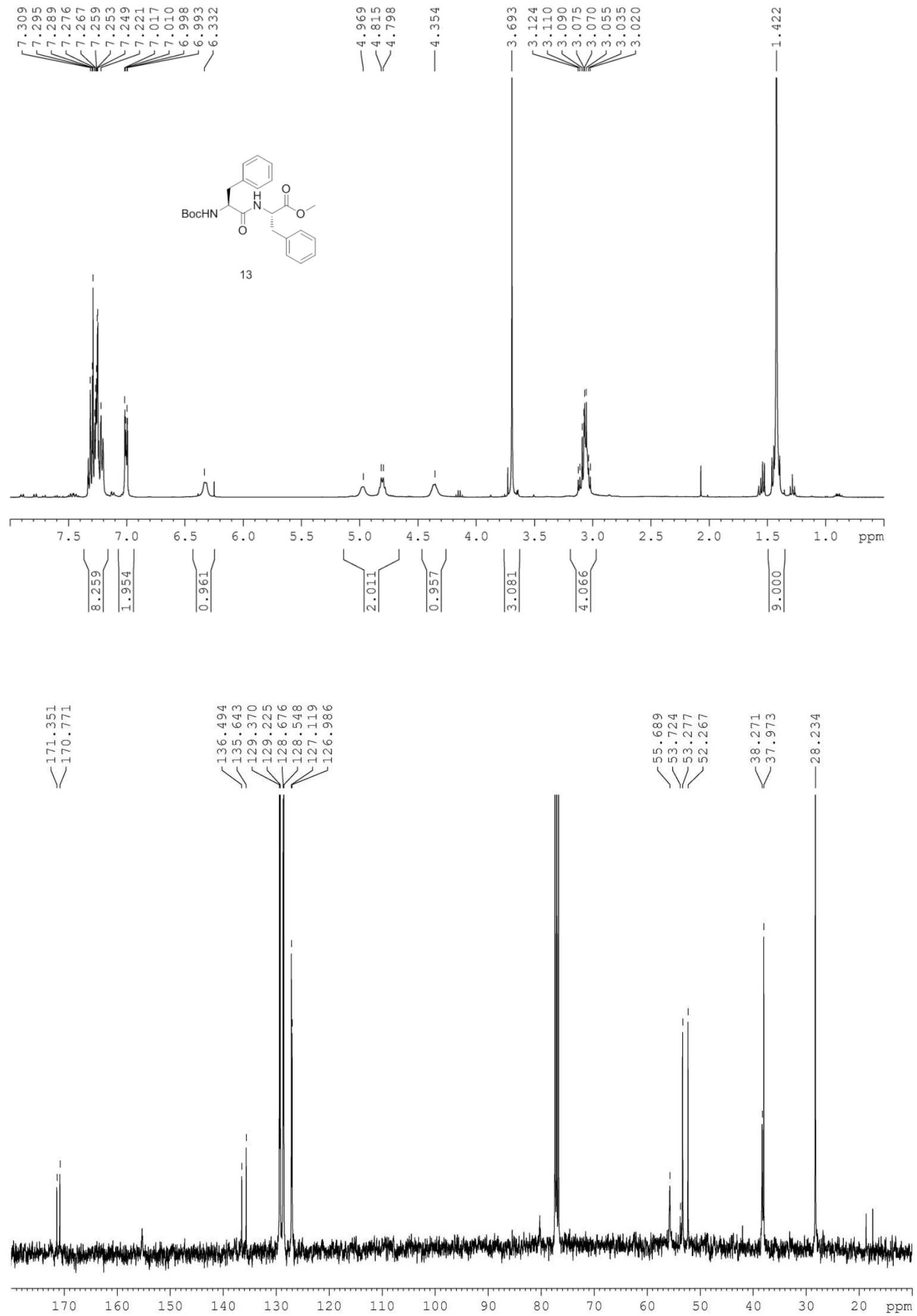

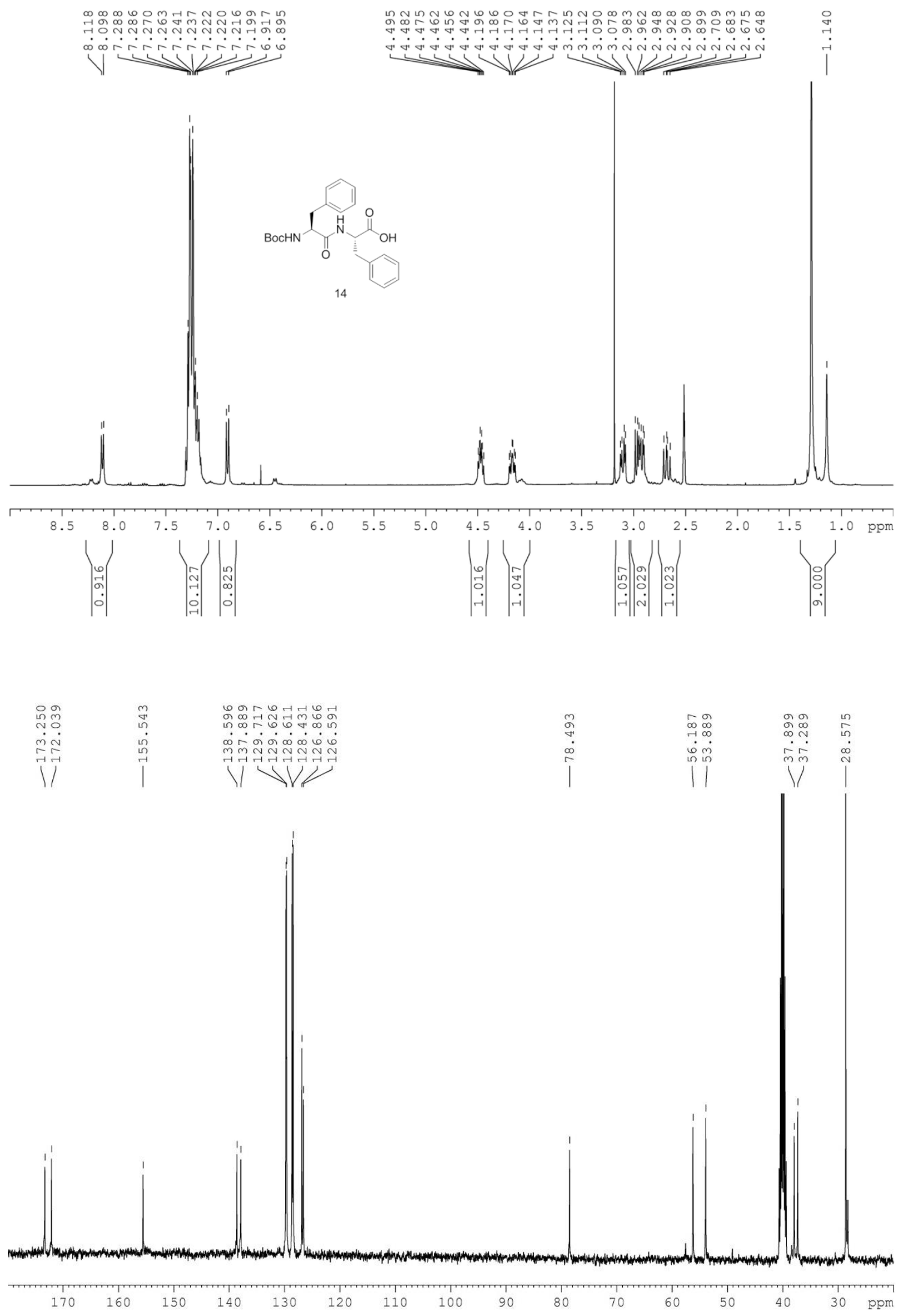
15.
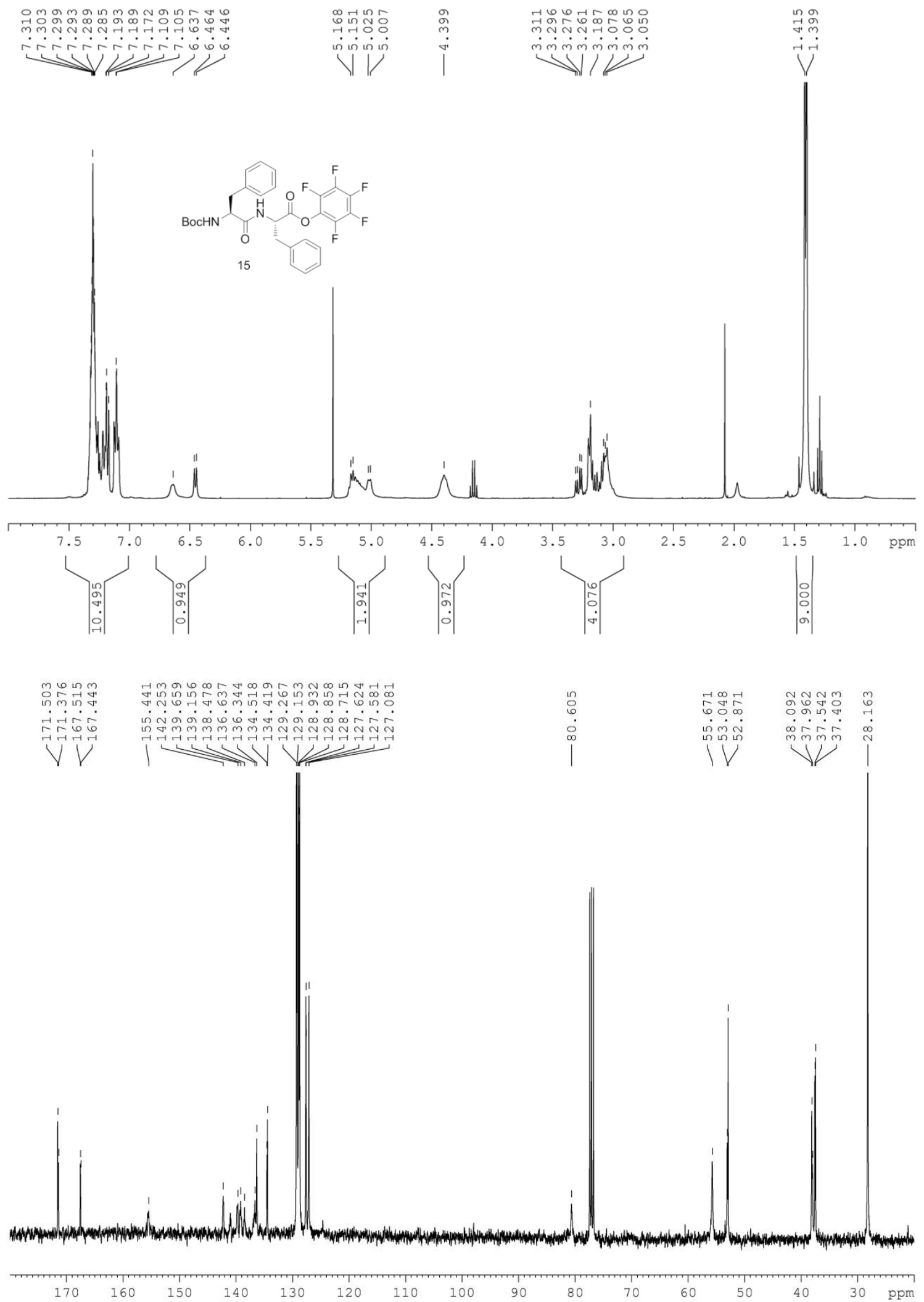
16.
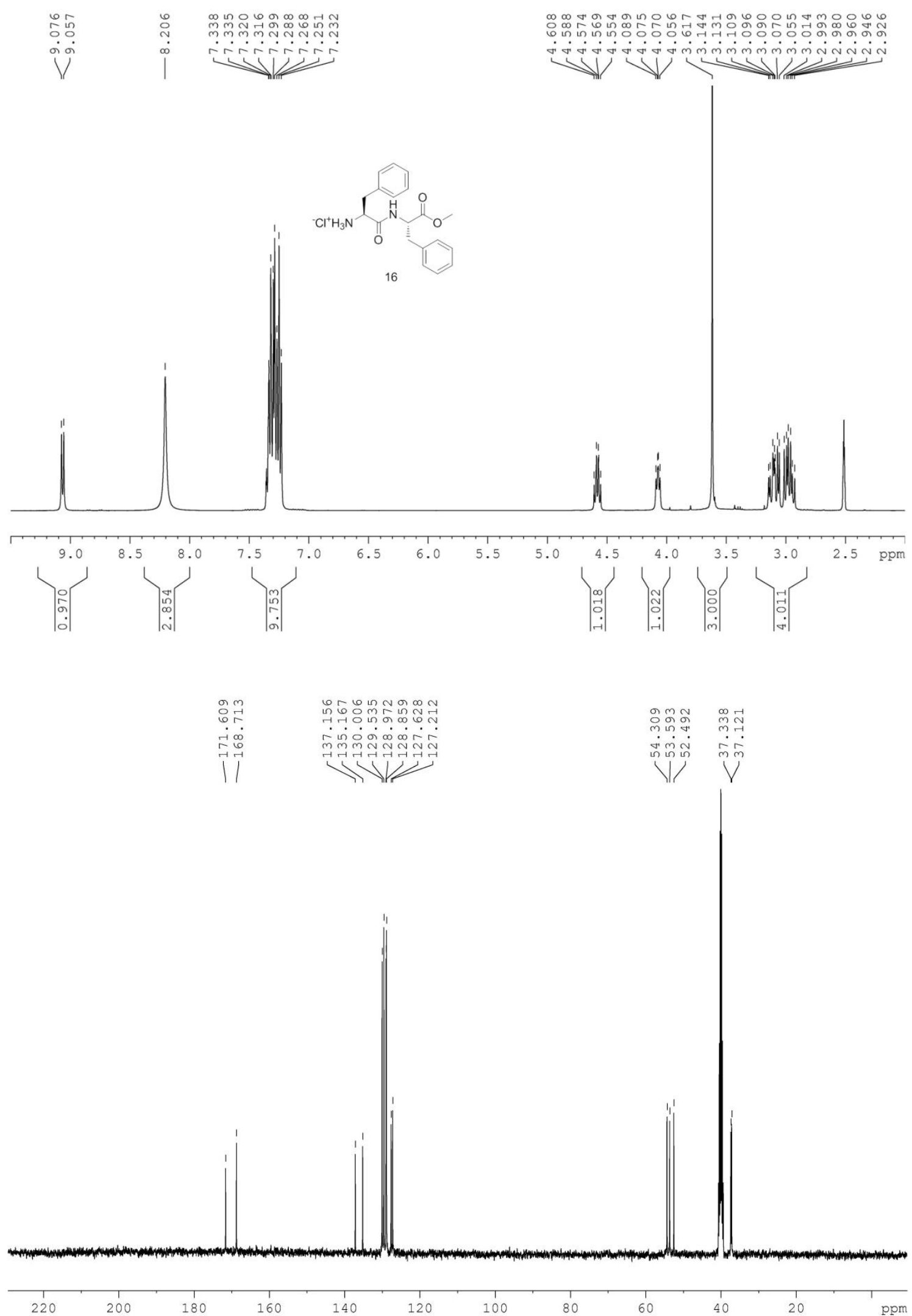
17.
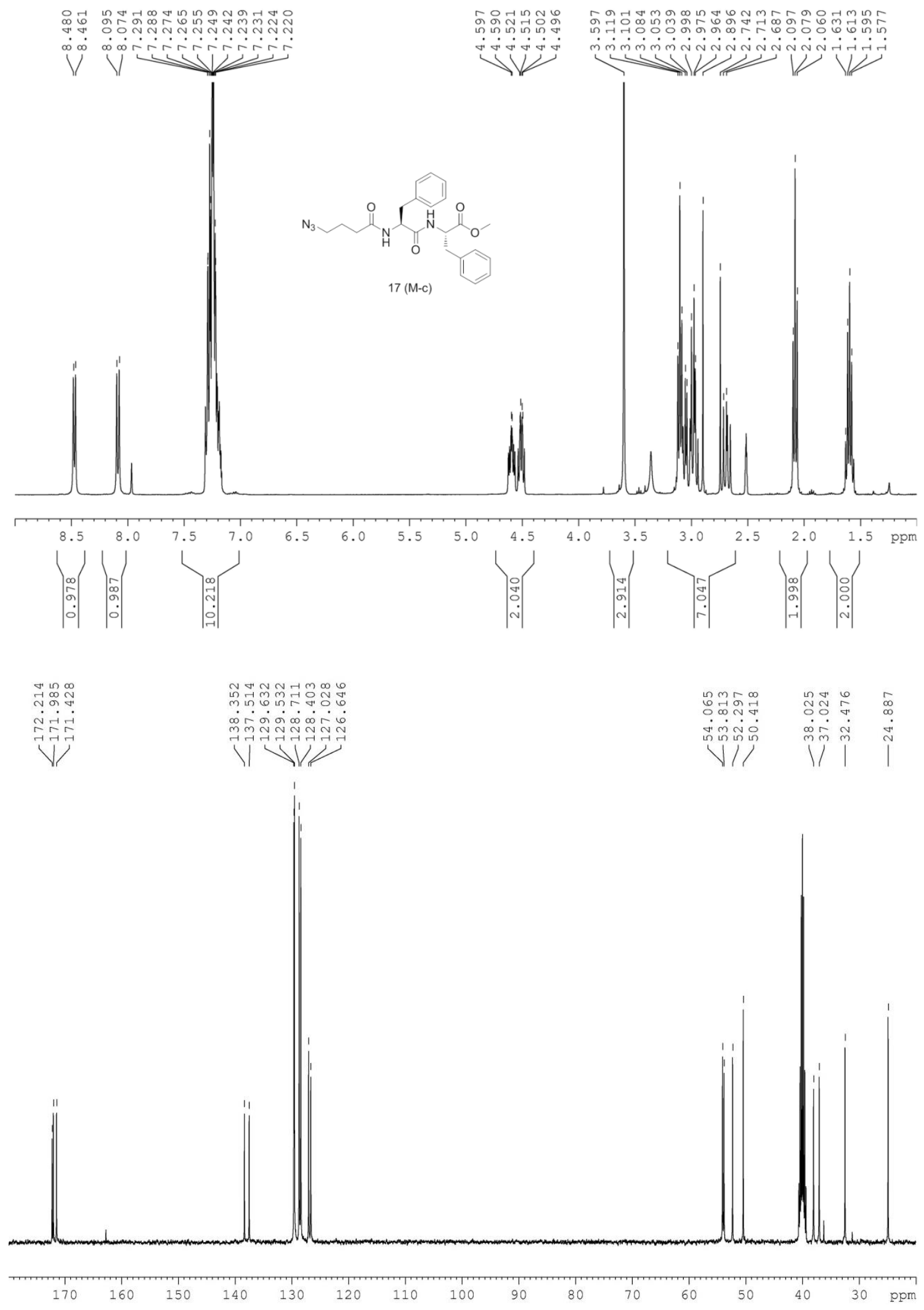
18.
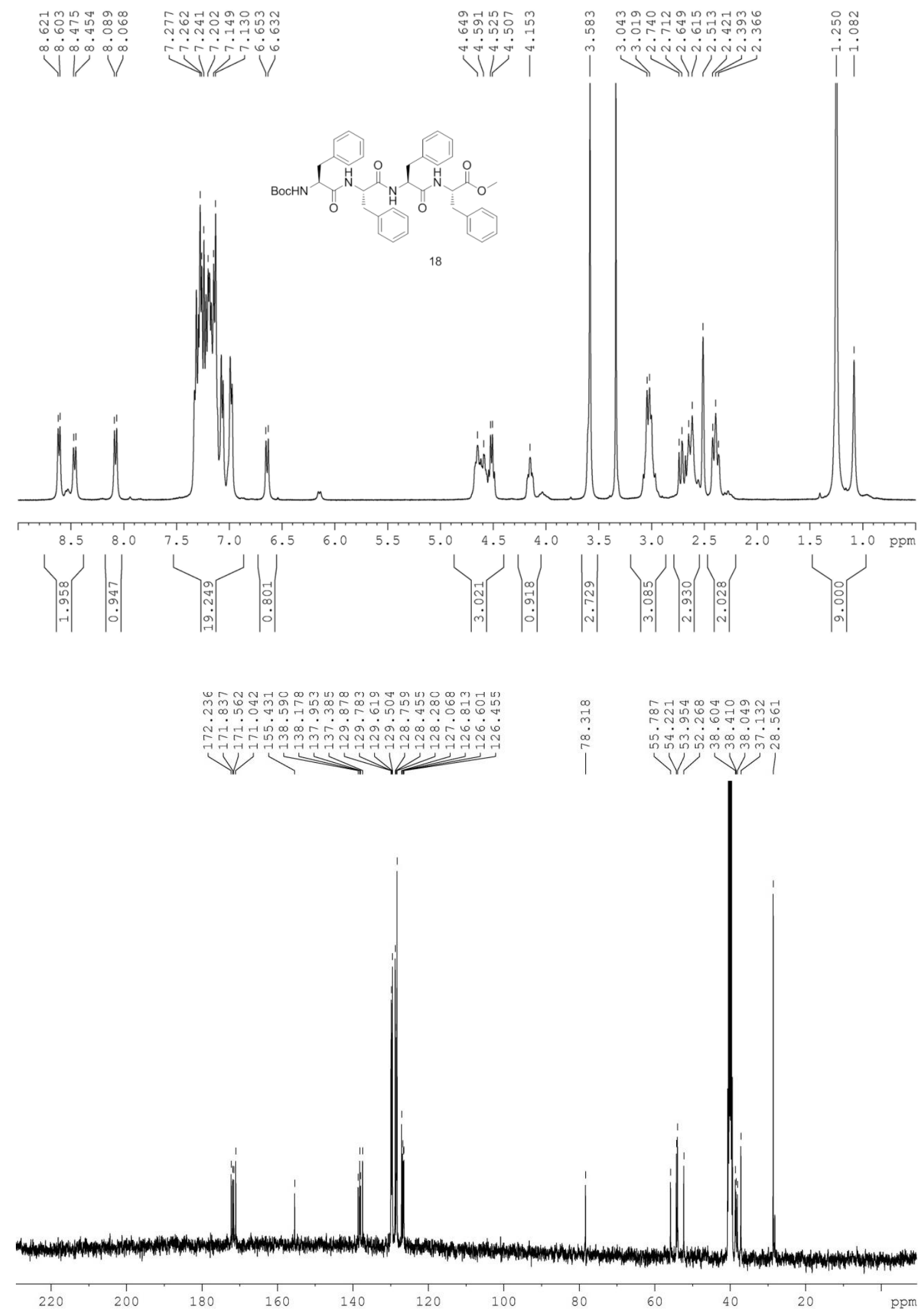
19.
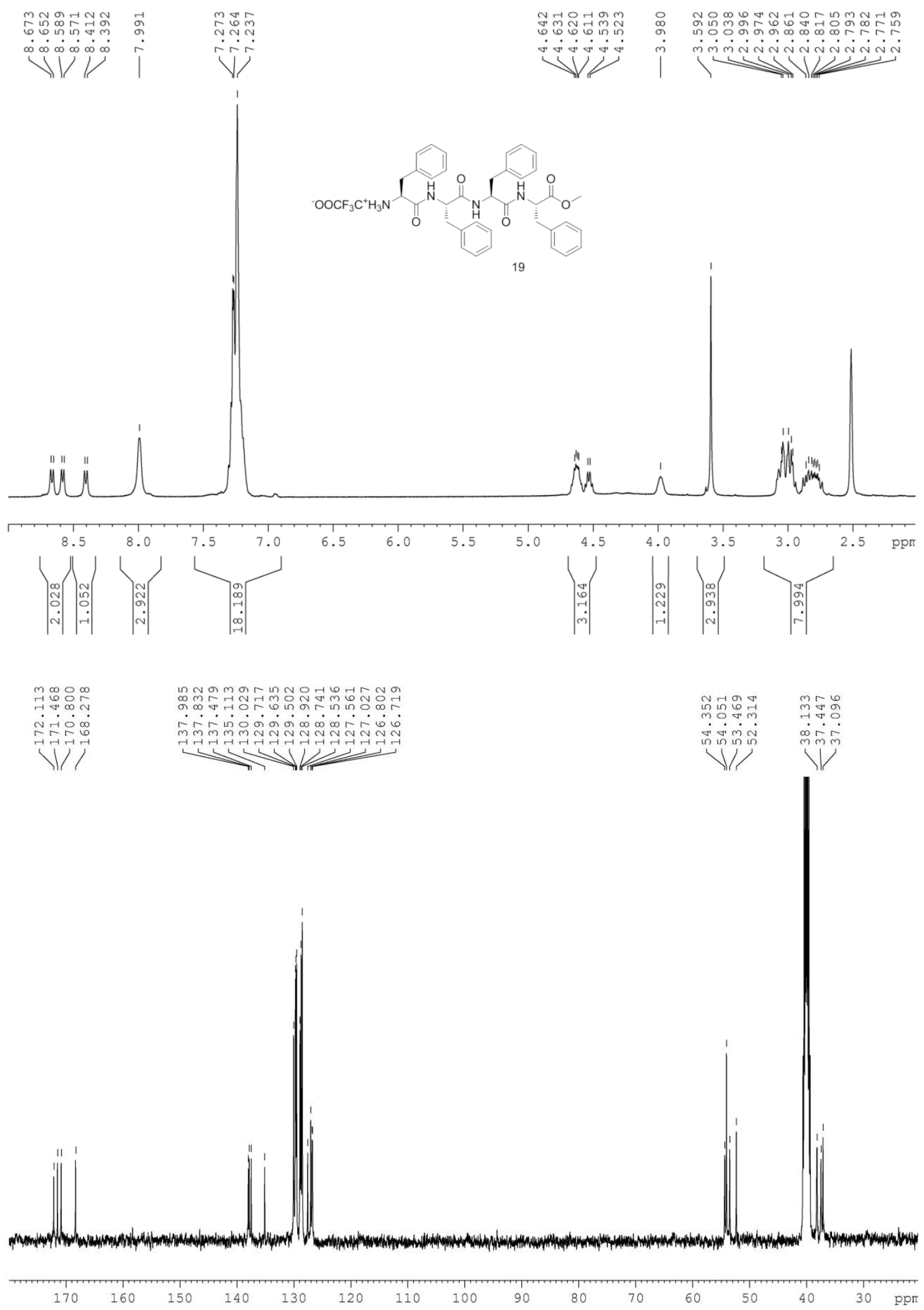
20.
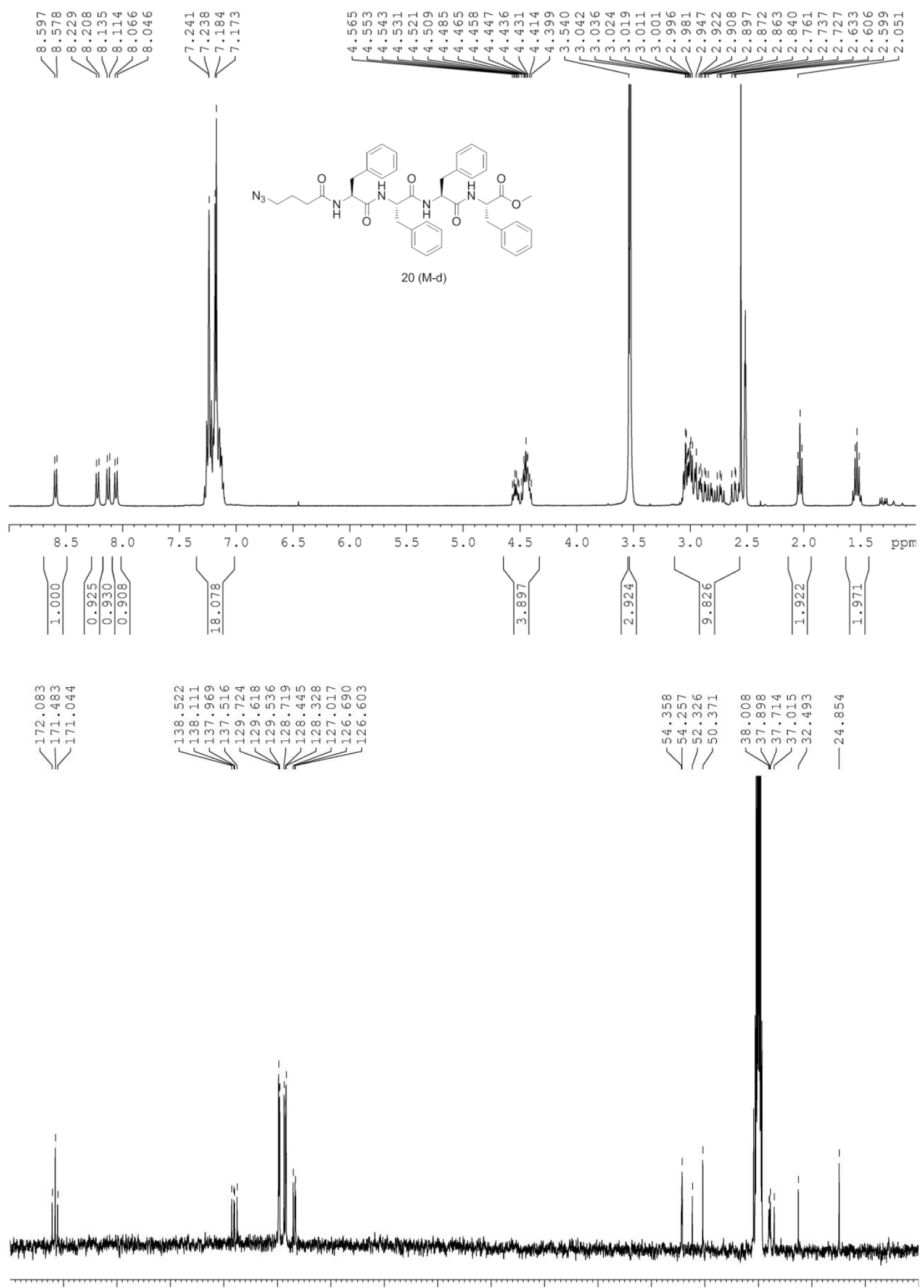
Tri-Mannose (AcO).
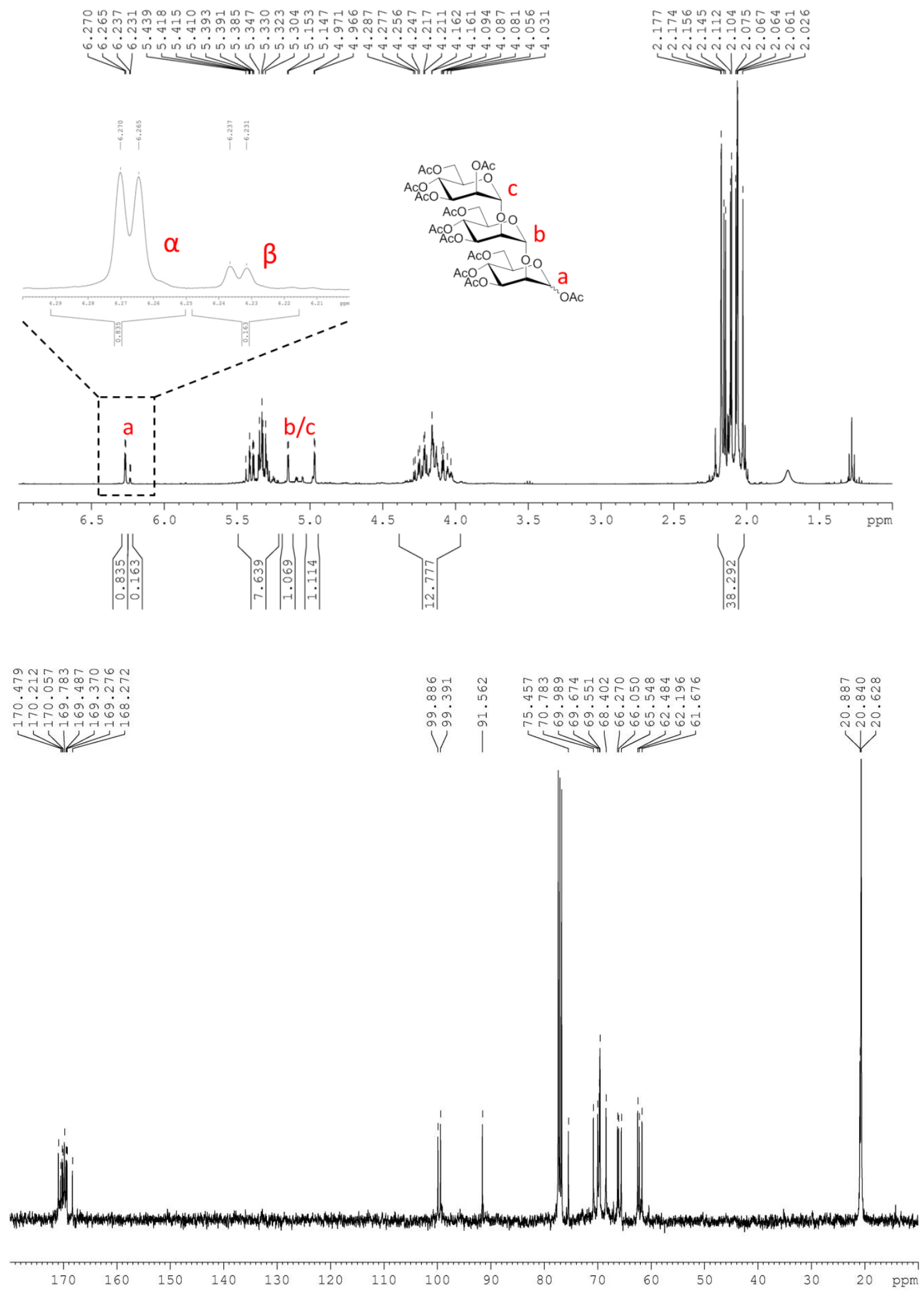
Tri-Mannose.
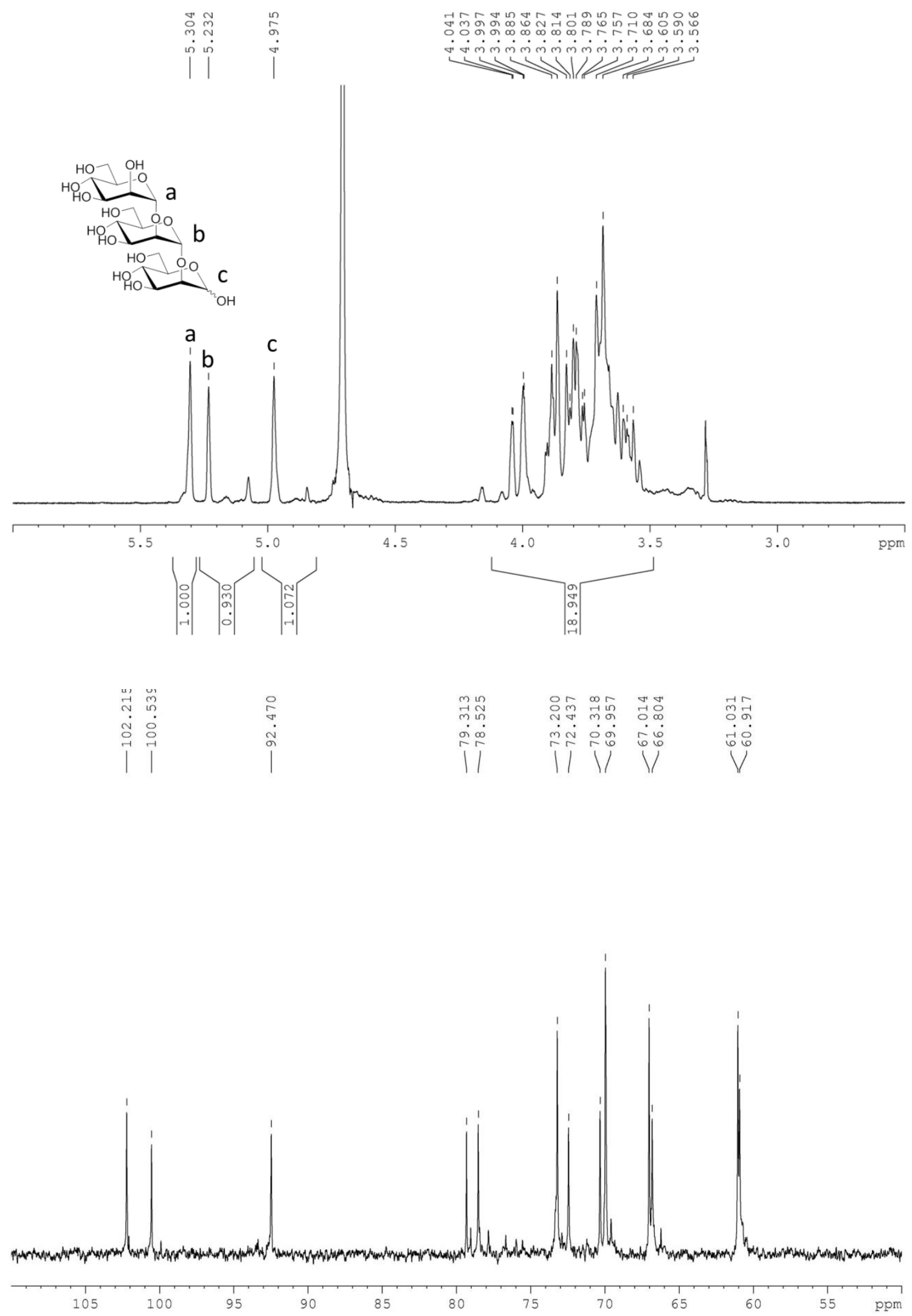
$\mathrm{P} 0-\mathrm{a}$

|

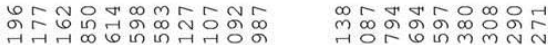

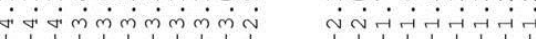

W V VI/ YIII

$\underbrace{\mathrm{O}}_{\mathrm{NHBOC}}$

PO-a

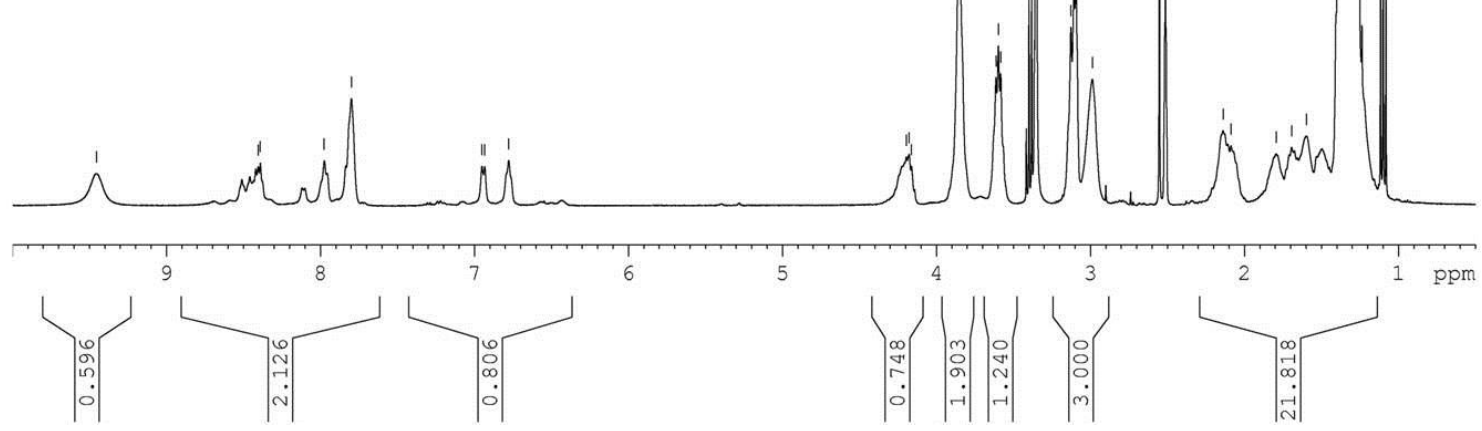

$\mathrm{P} 0-\mathrm{b}$

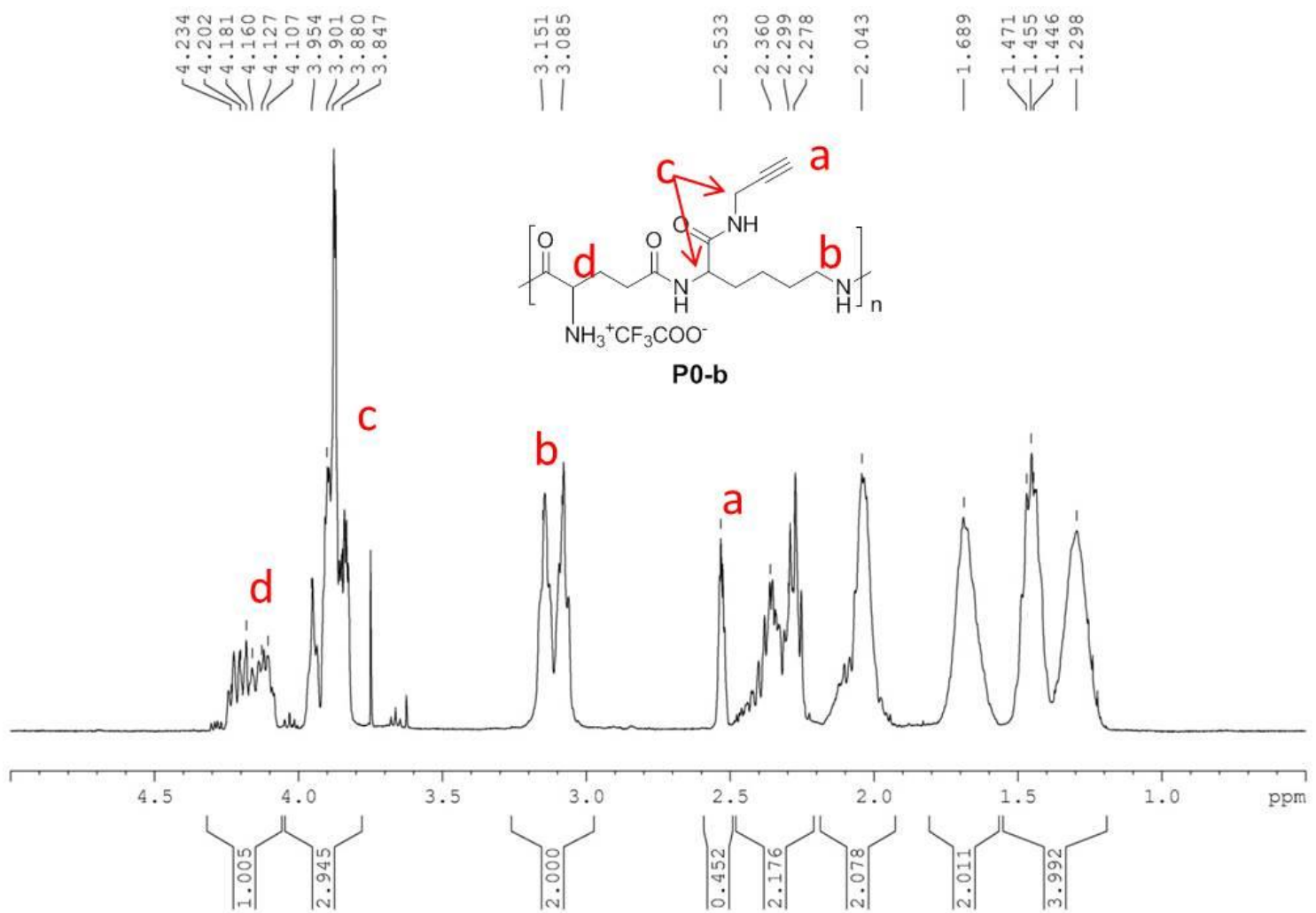


$\mathrm{P} 0-\mathrm{c}$

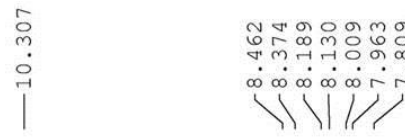

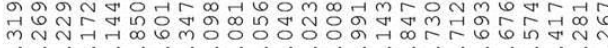

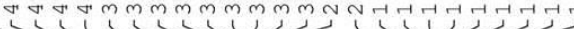
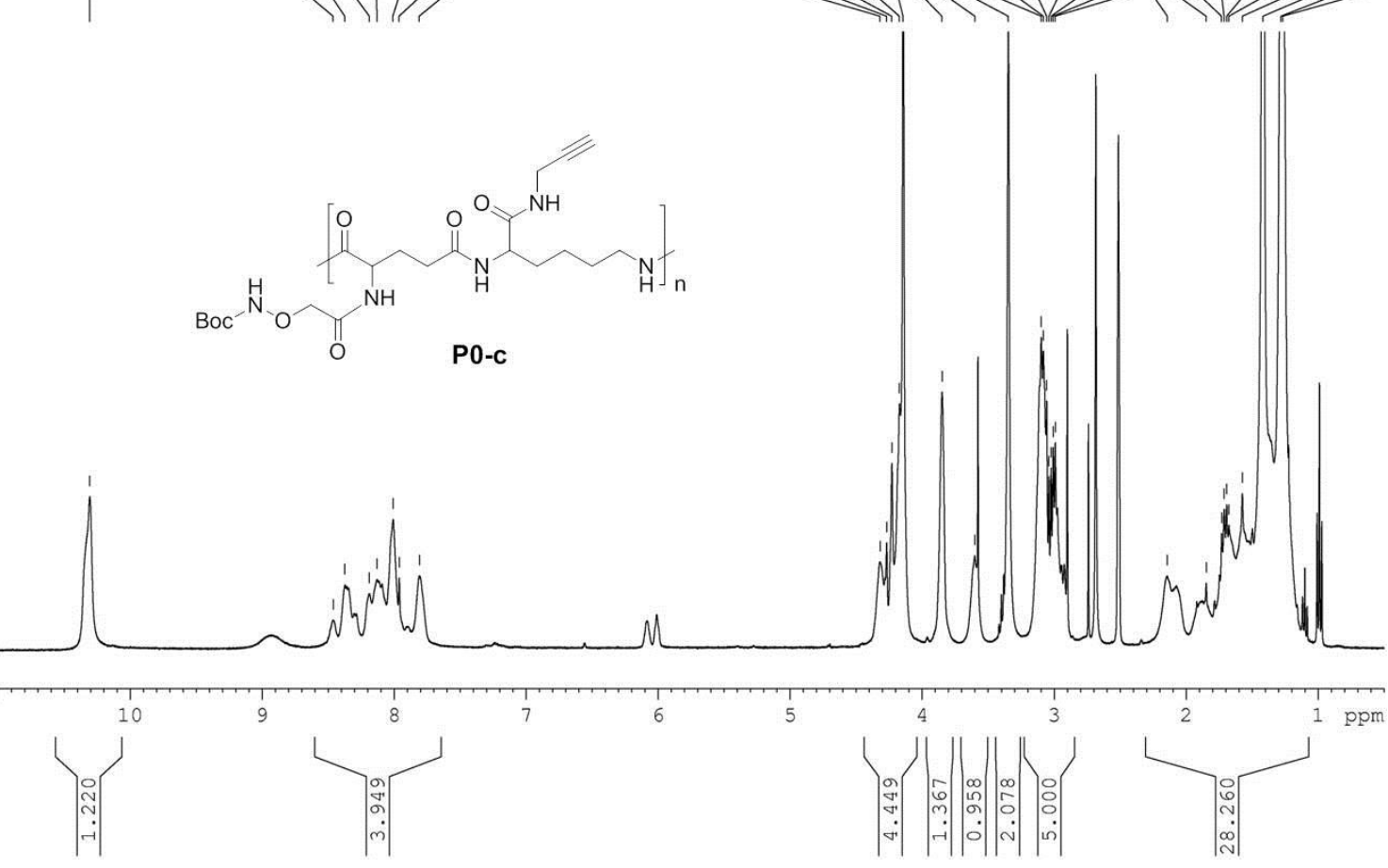

$\mathrm{P} 0$

||

\ֶं

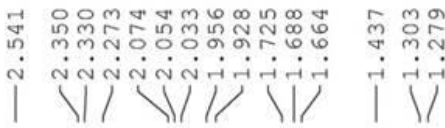


P1-a

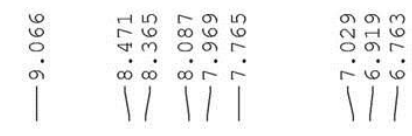

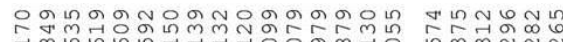

C $_{\mathrm{NH}}^{O}$

BocHN $N$ NBBoc

P1-a

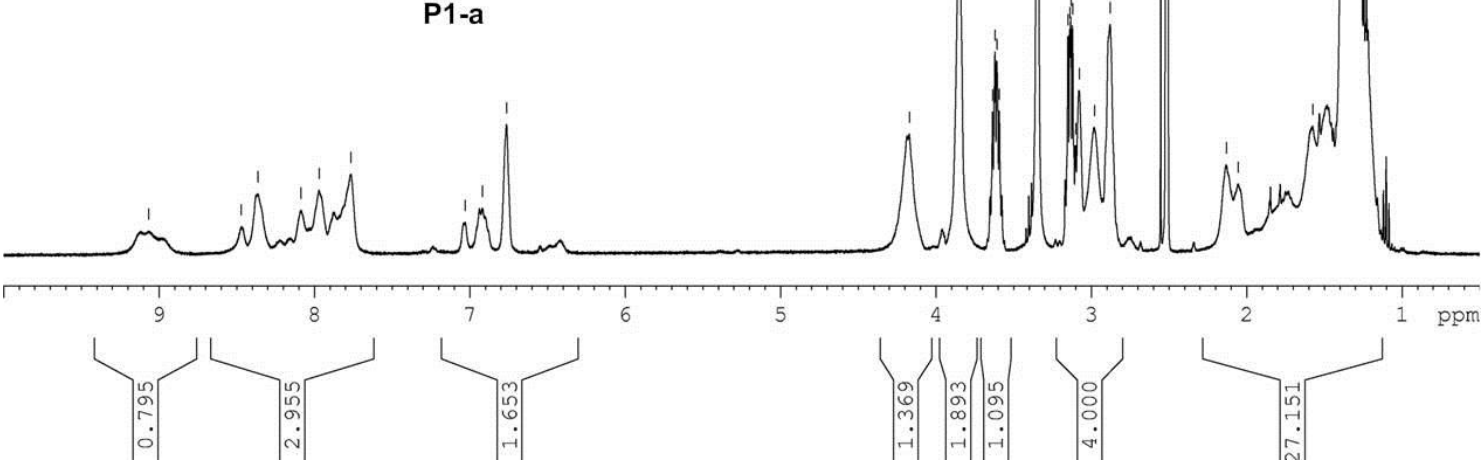

P1-b

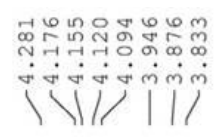

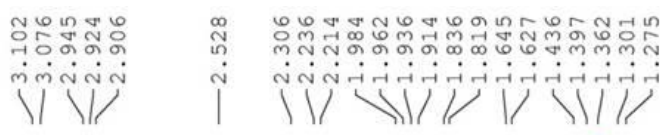

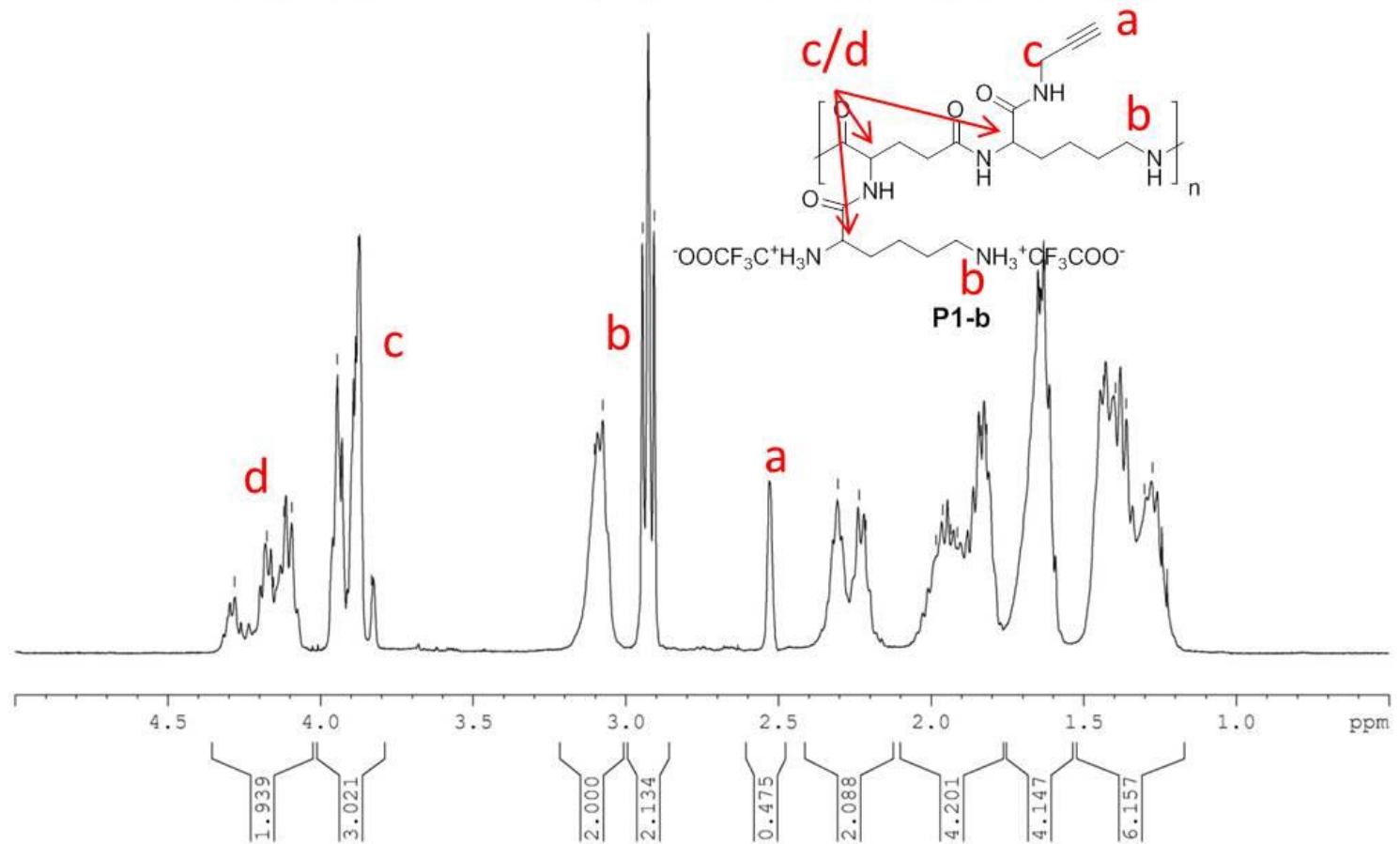


P1-c

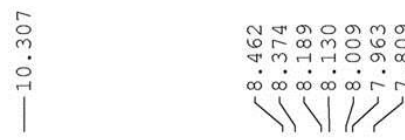

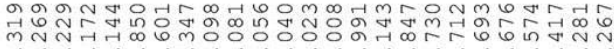

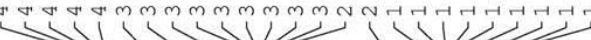

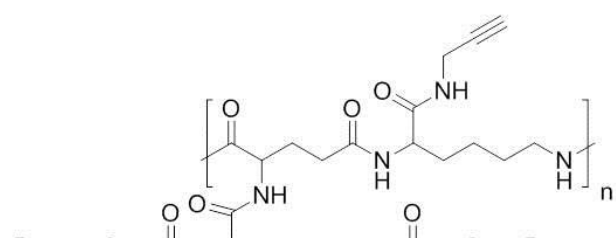

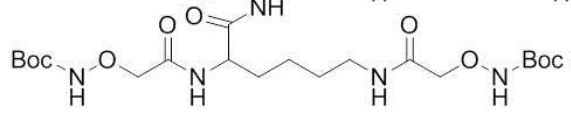

P1-c
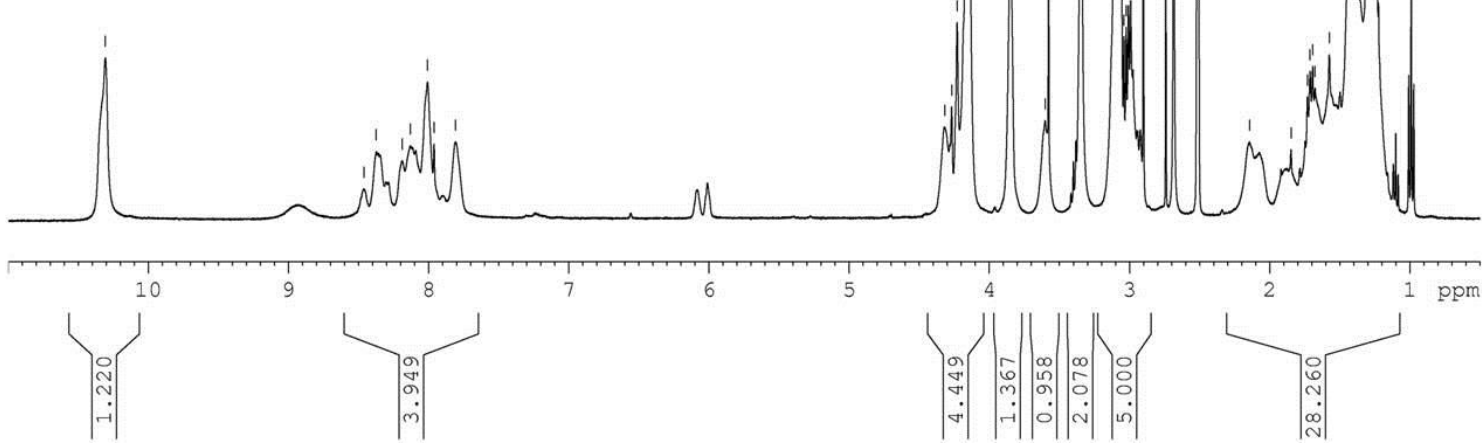

P1

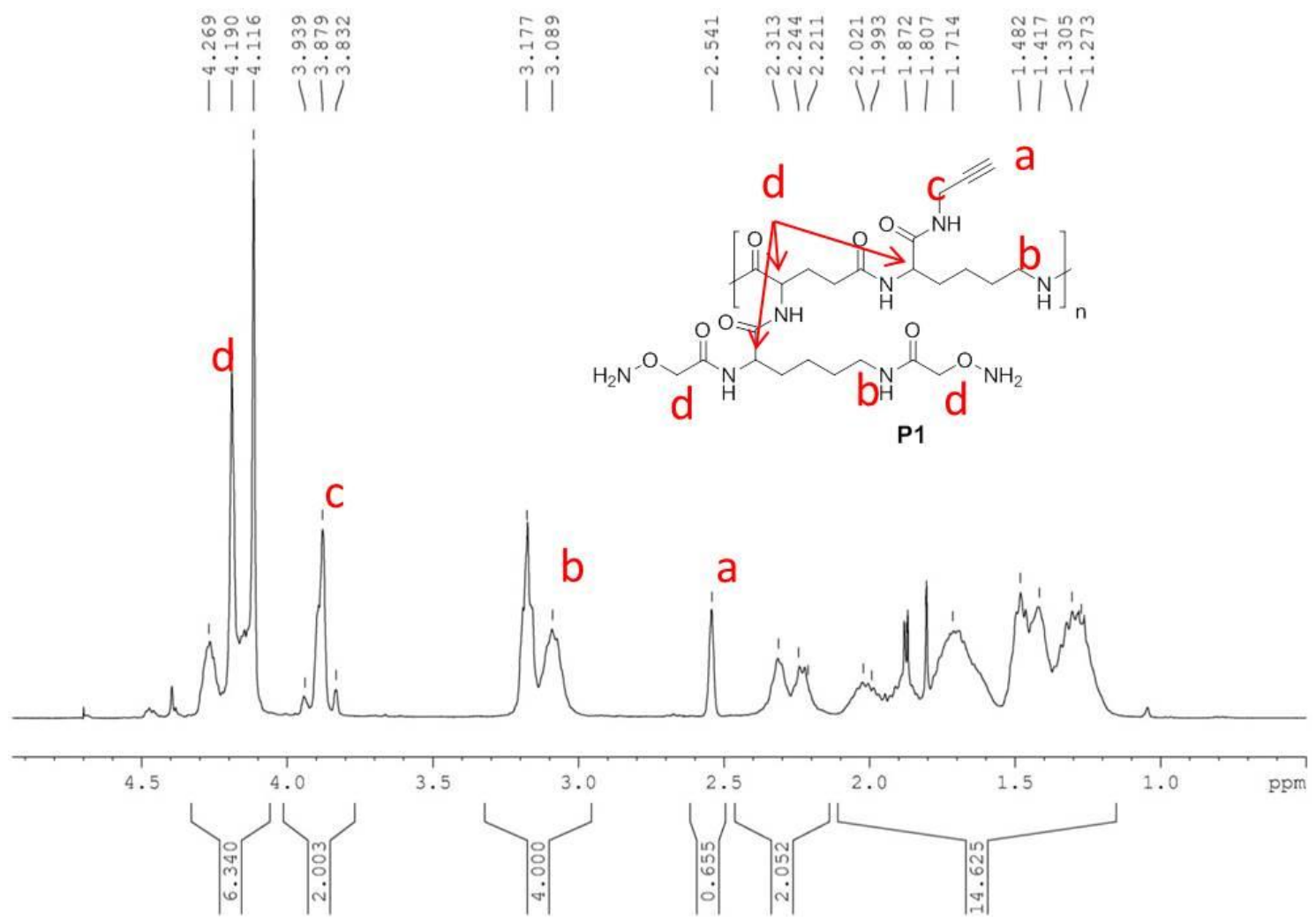


P2-a

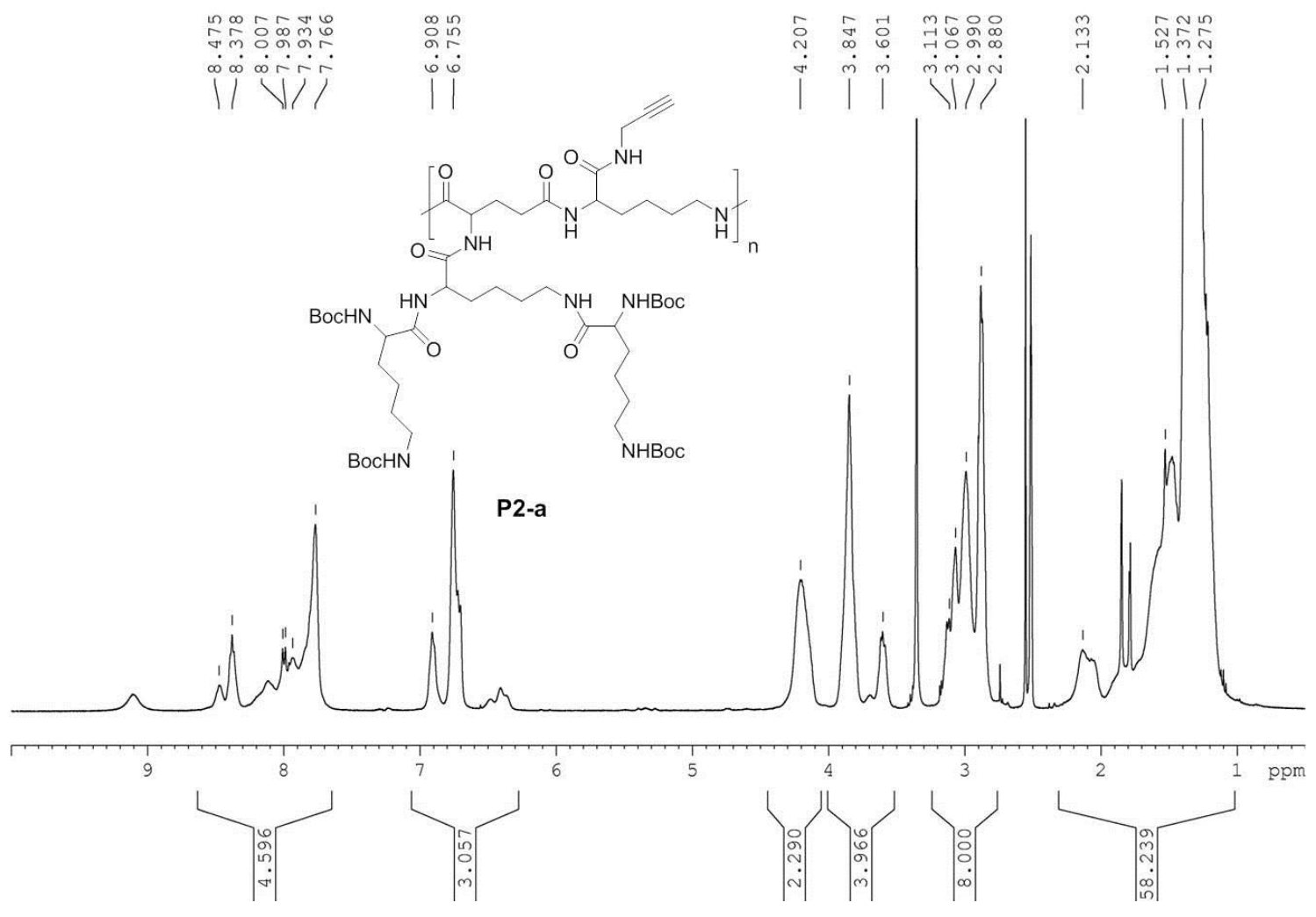

P2-b

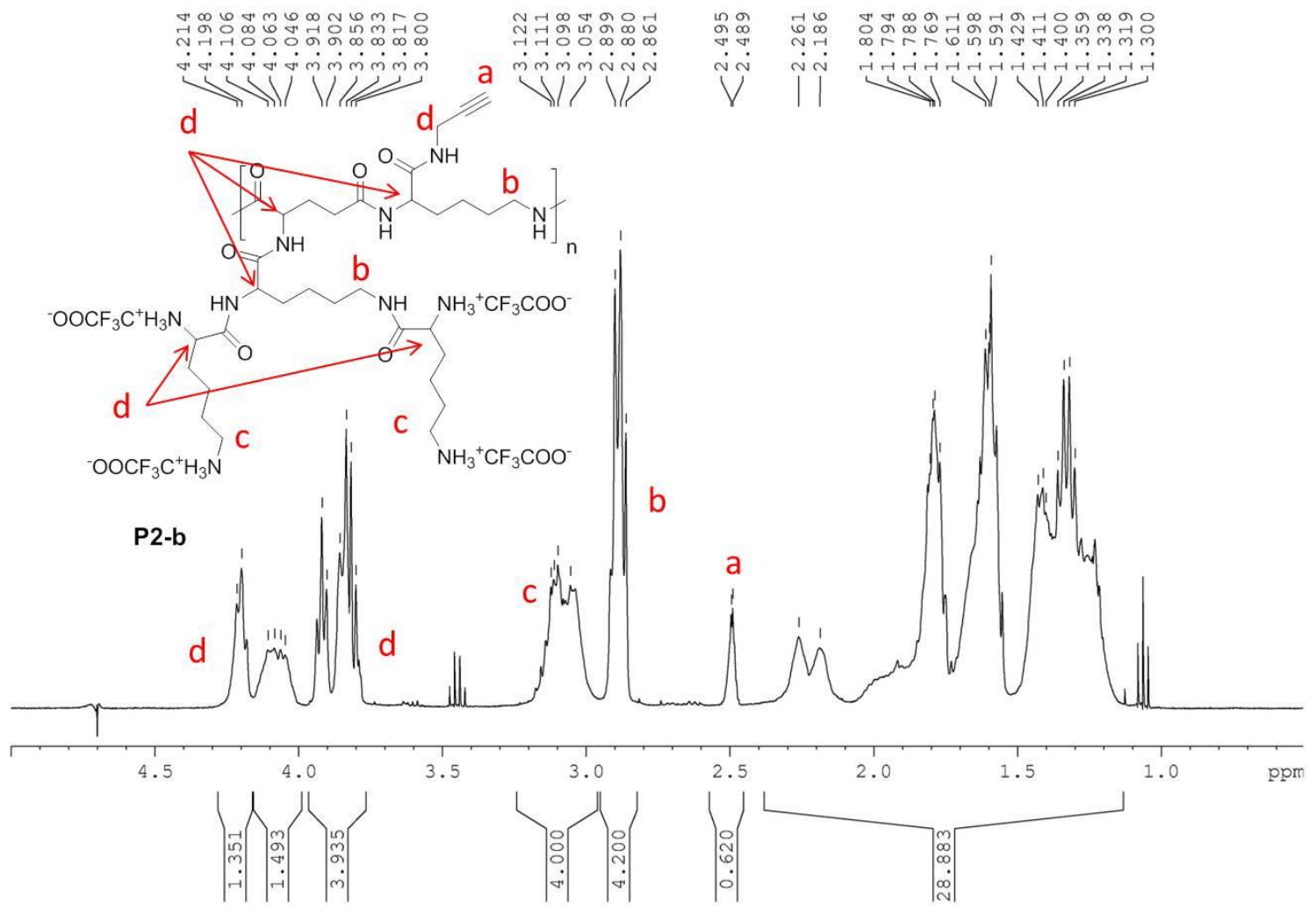


P2-c

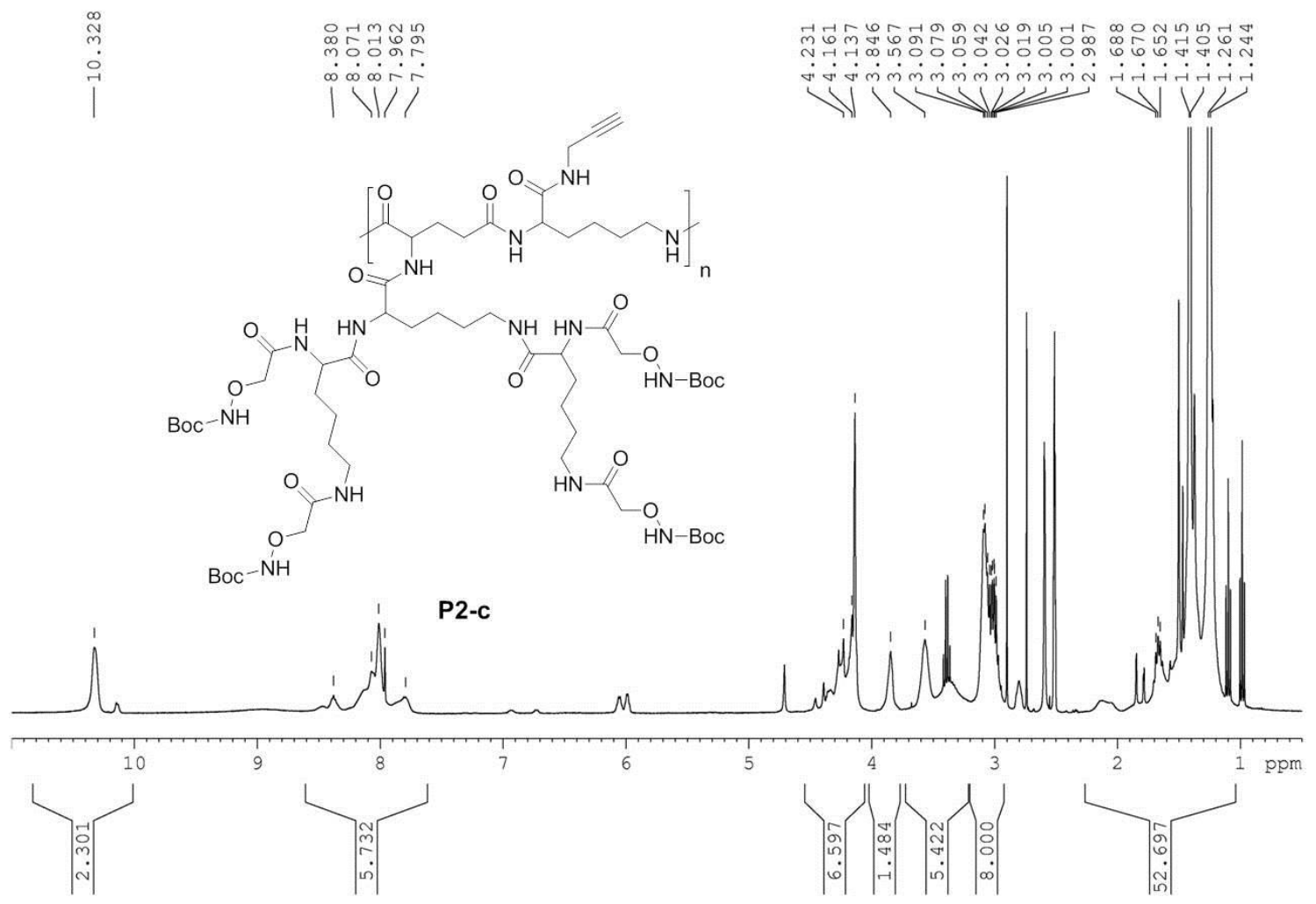

P2

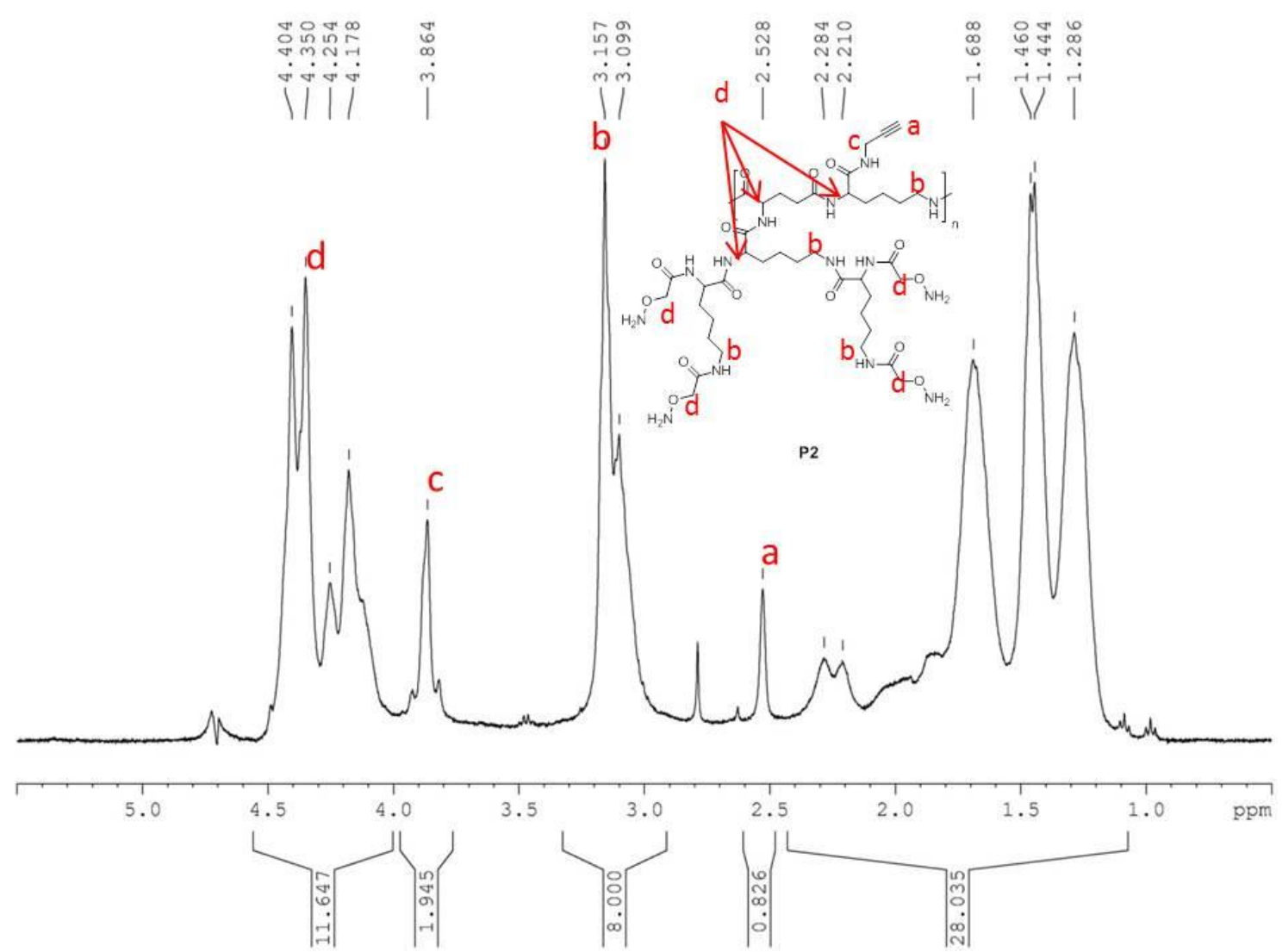




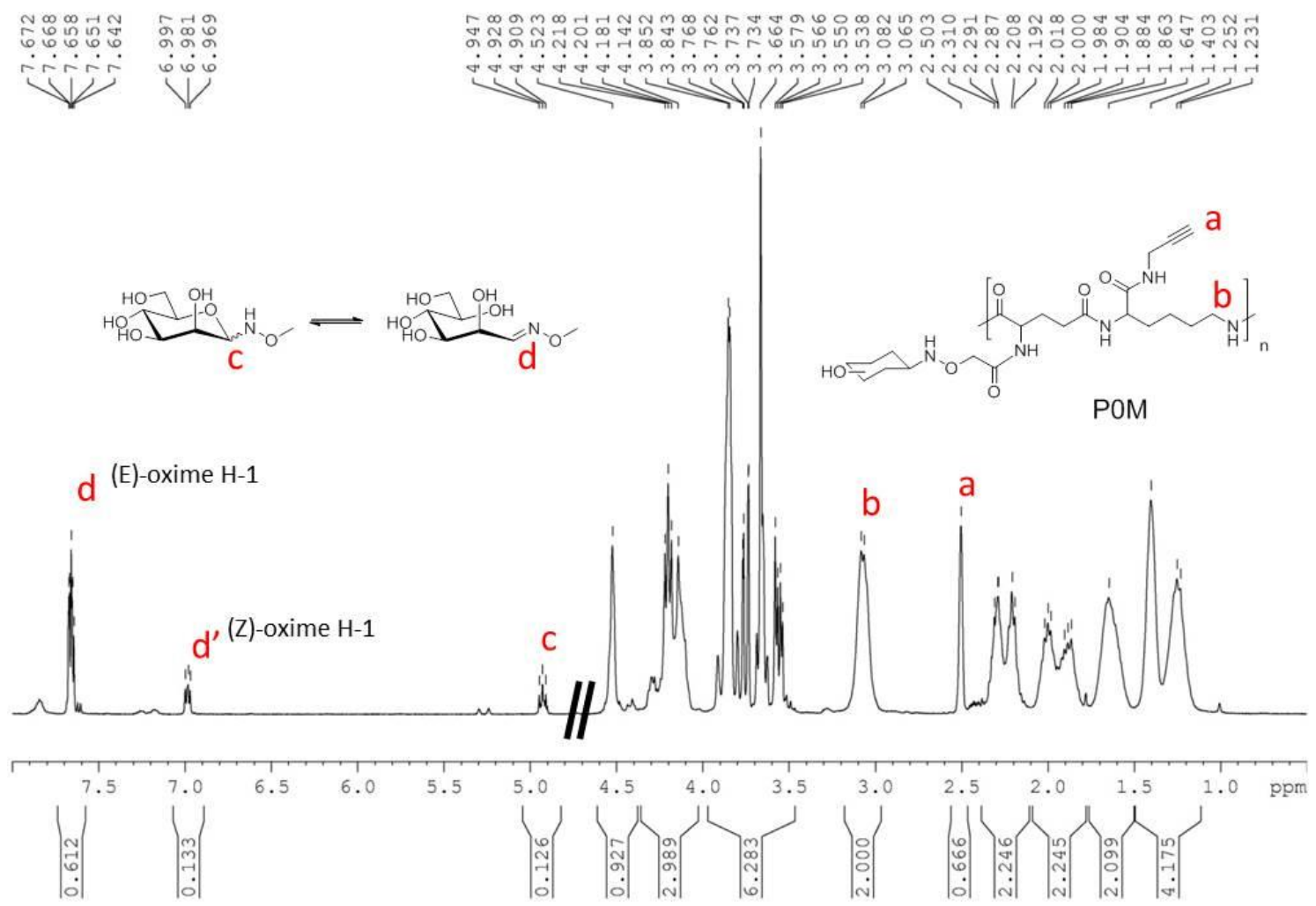

P1M

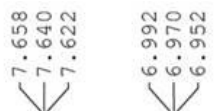

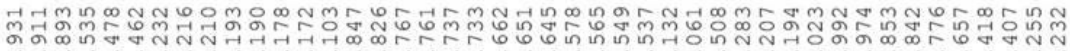
耳. $\underbrace{C}=\frac{d}{d O^{2}}$ d (E)-oxime $\mathrm{H}-1$

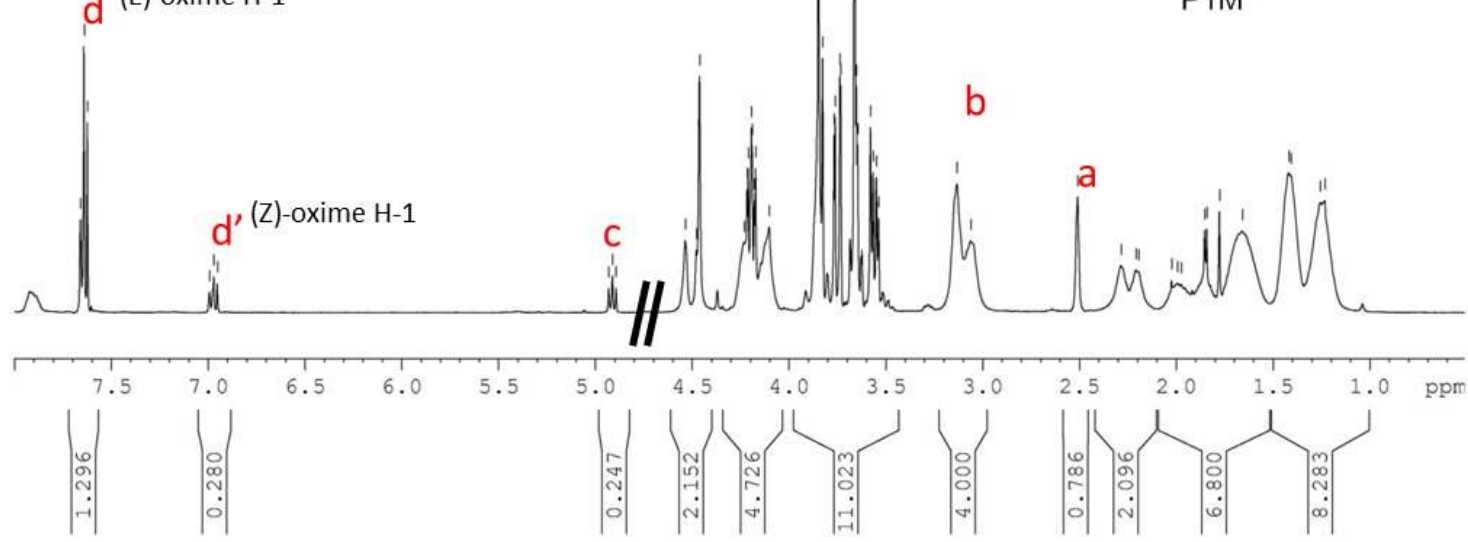




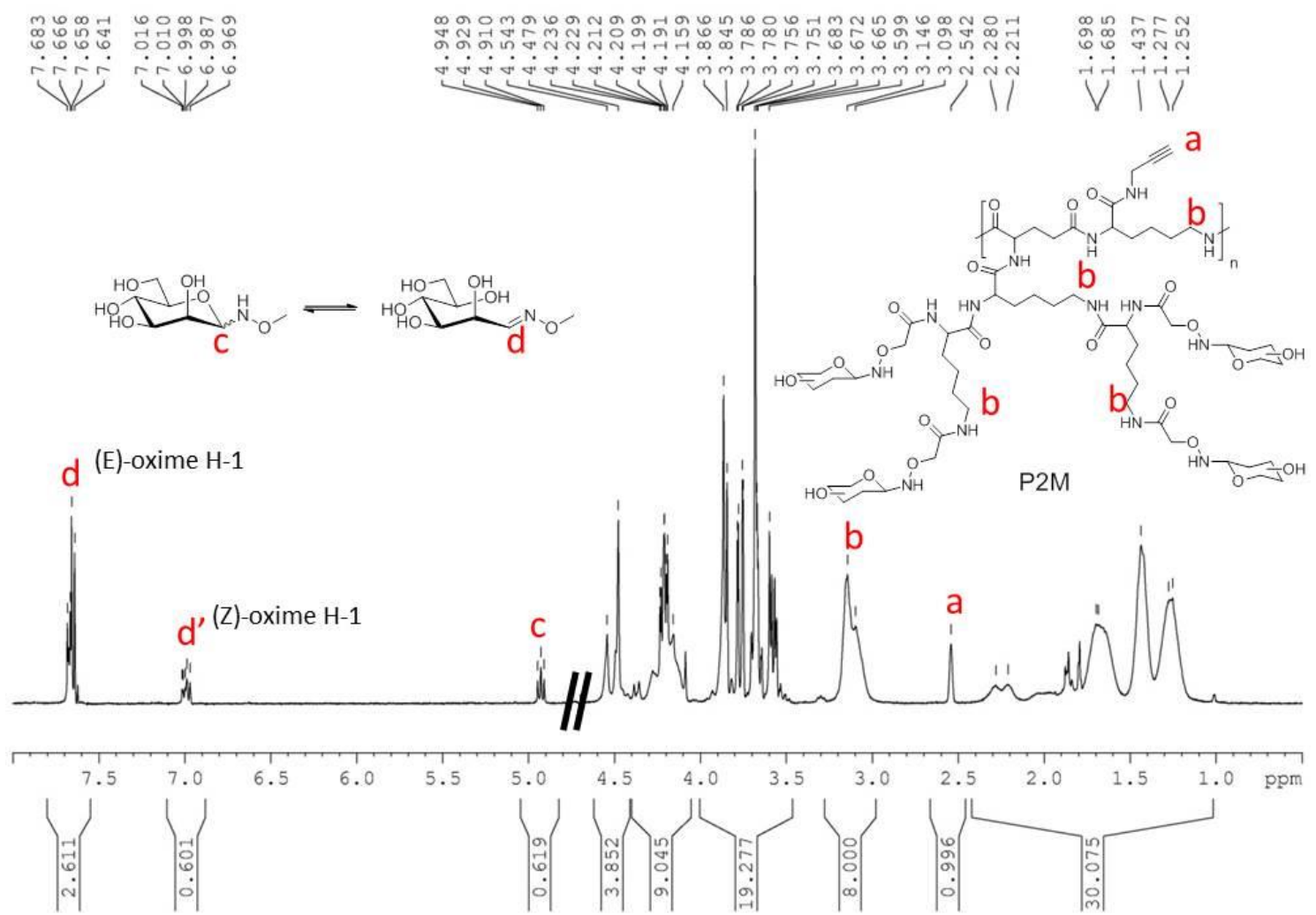

POdM

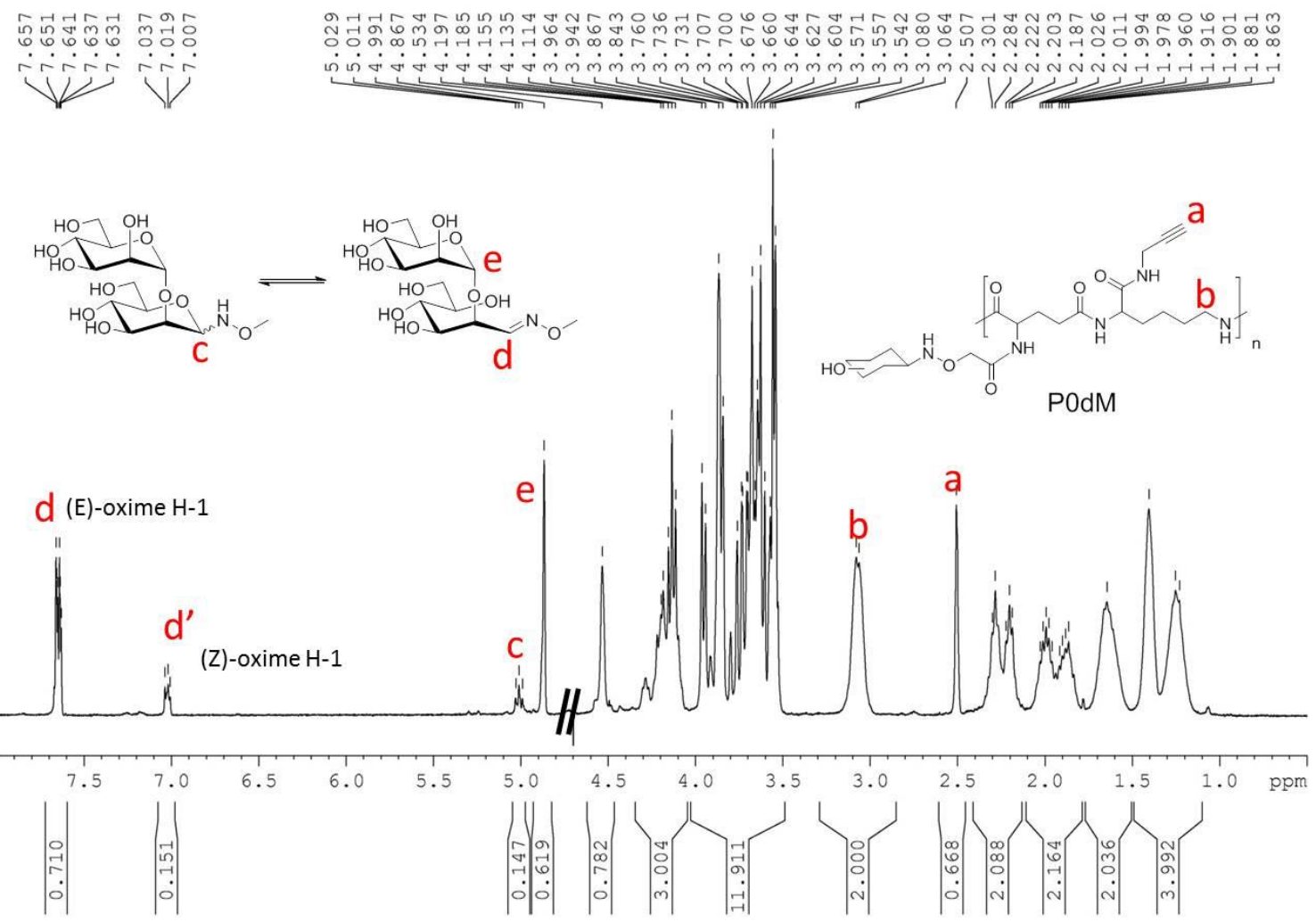


P1dM

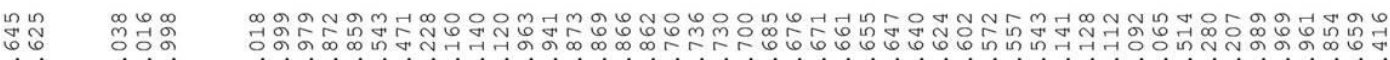

ij
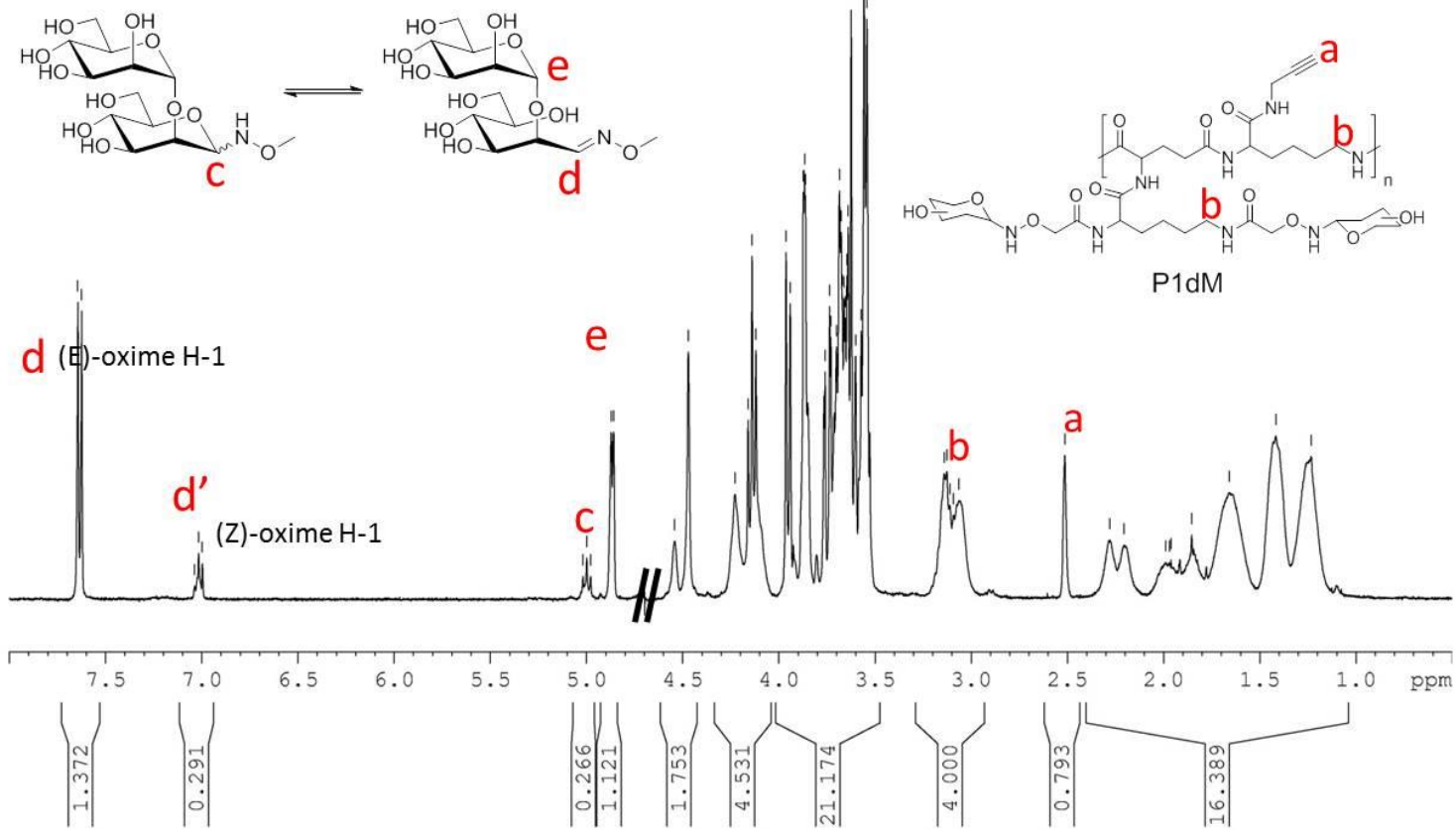

\section{P2dM}

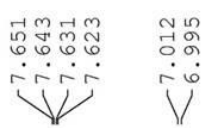
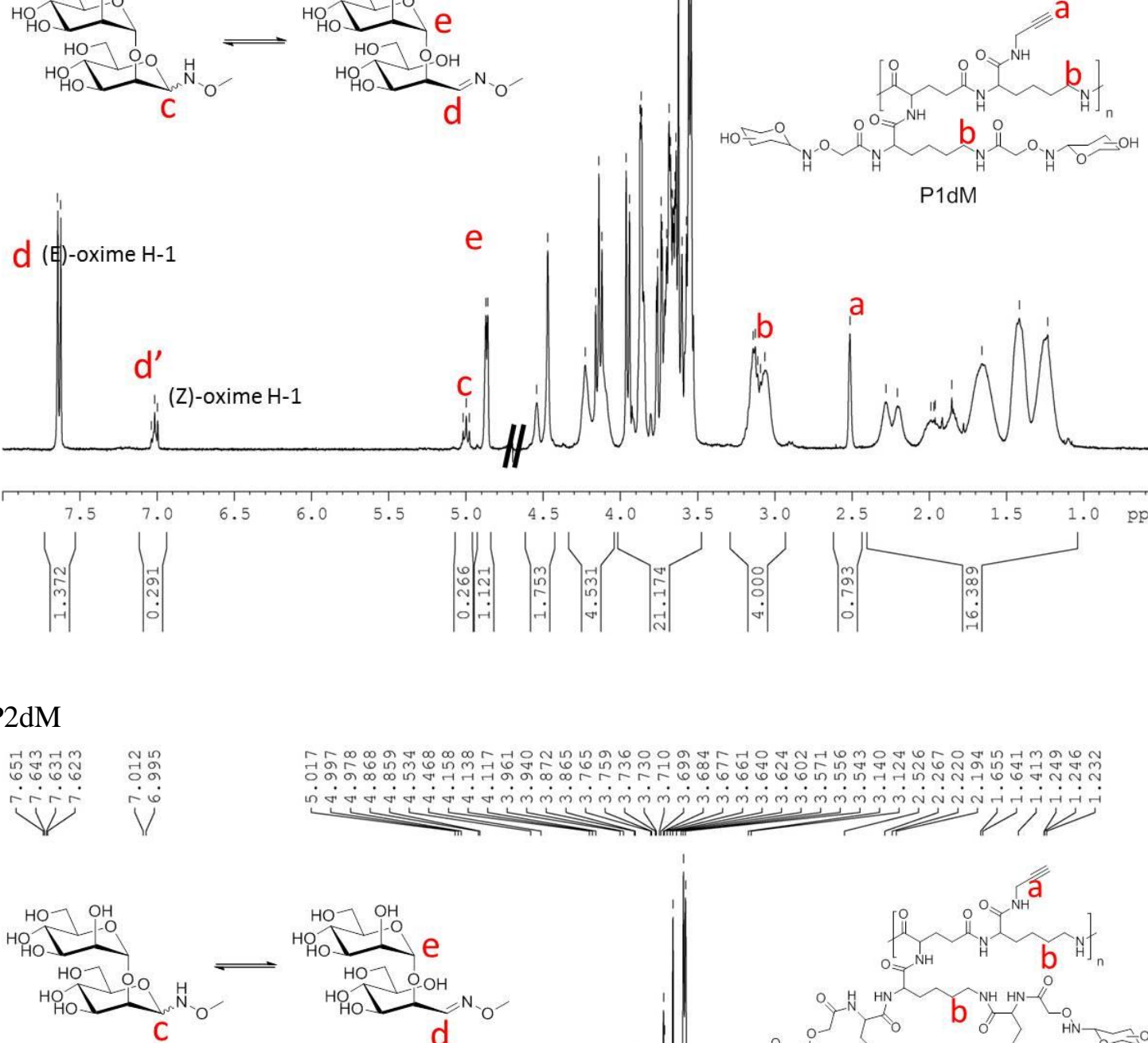
P0tM

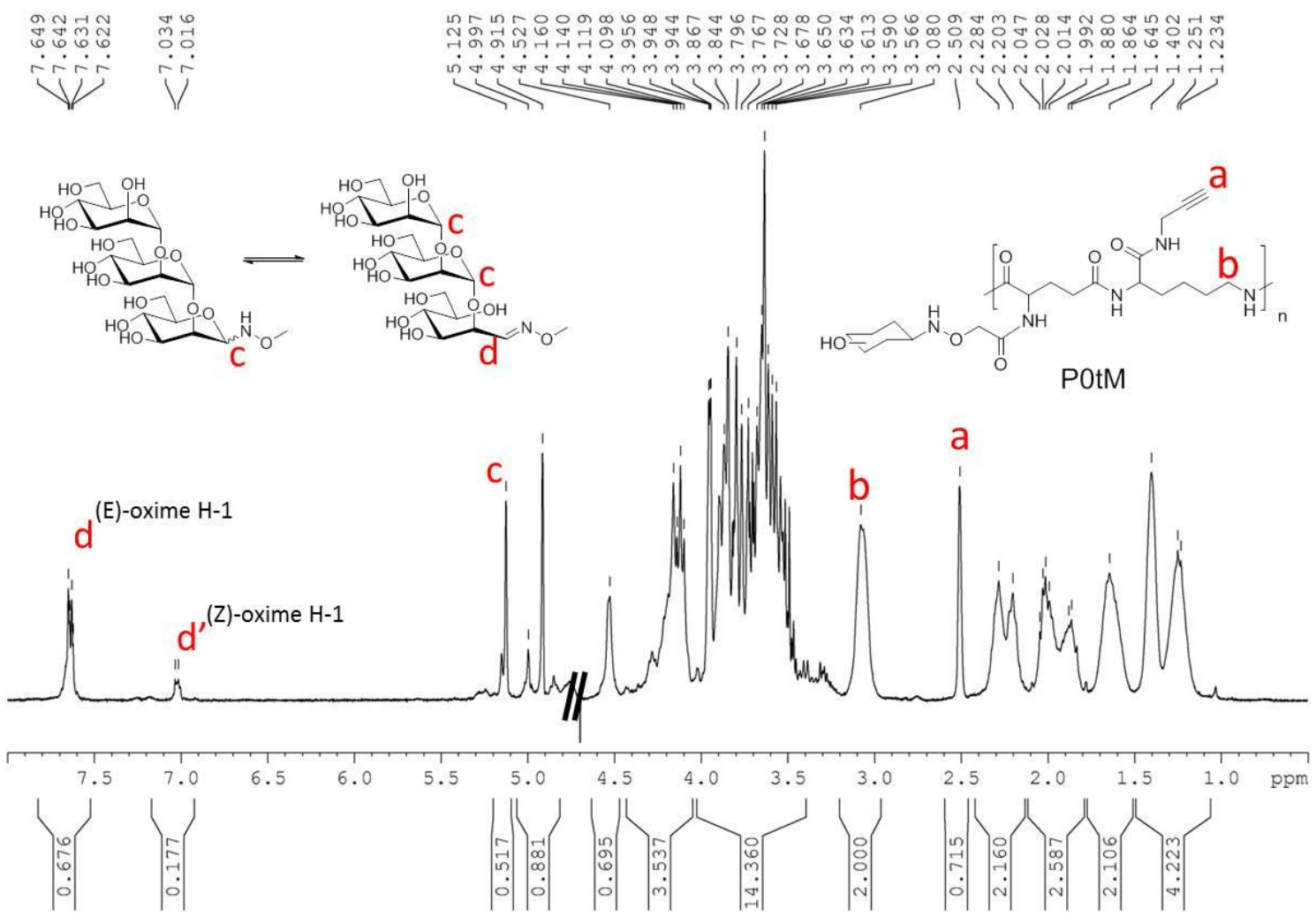

P1tM

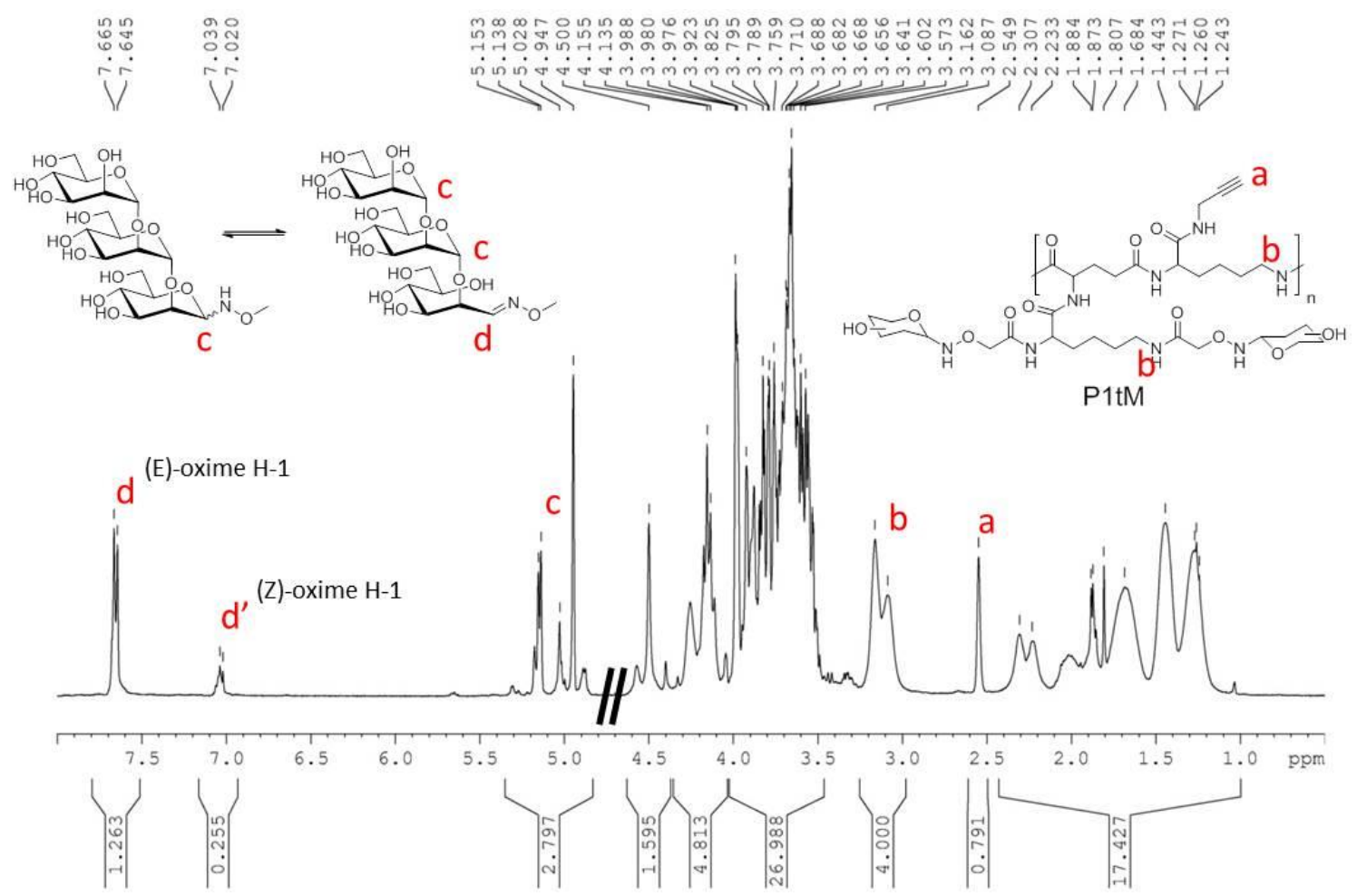


P2tM

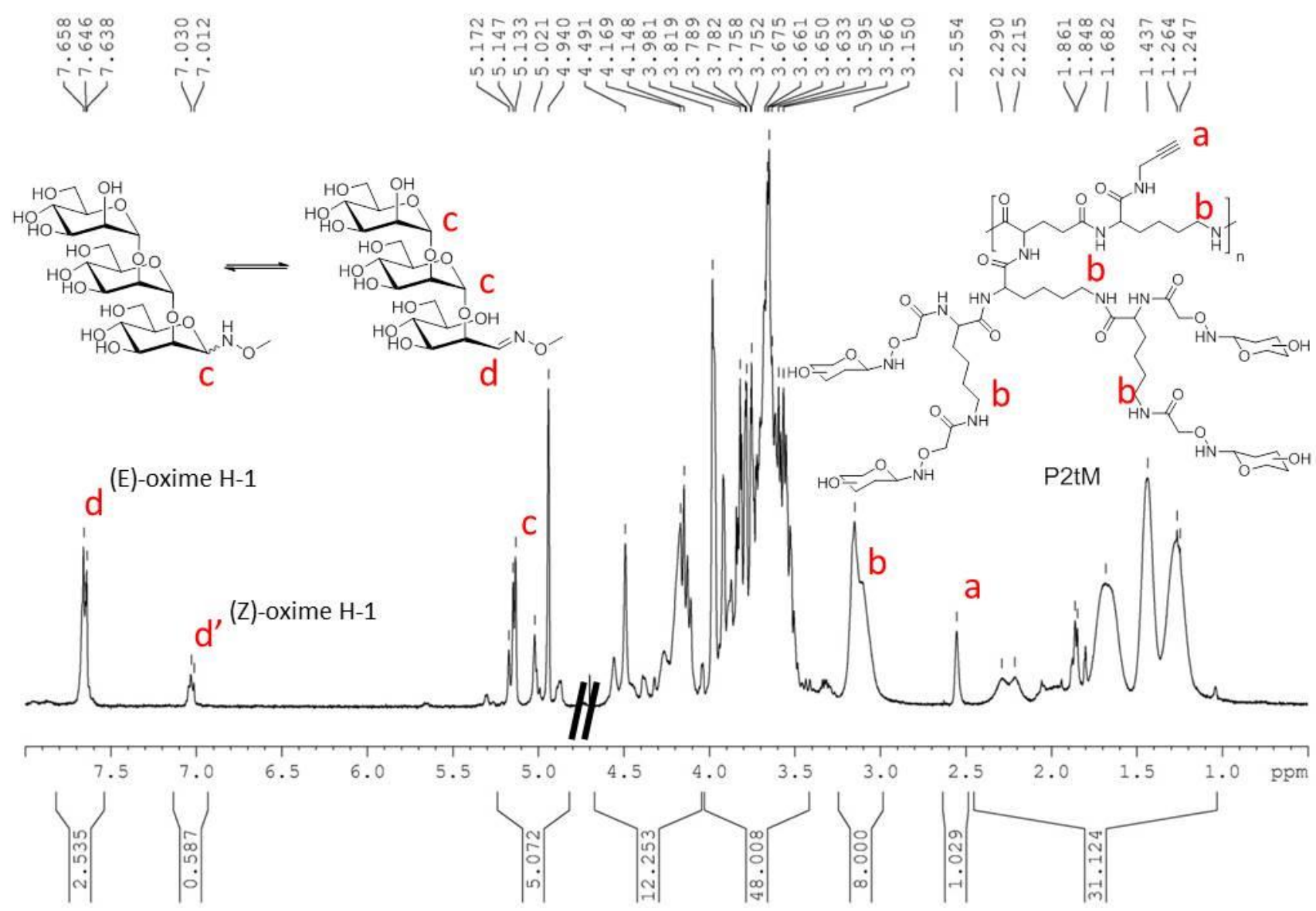




\section{Reference:}

(1) (a) Szurmai, Z.; Jánossy, L.; Szilágyi, Z.; Vékey, K., J. Carbohydr. Chem. 1998, 17, 417-437; (b) Szurmai, Z.; Jánossy, L.; Carbohydr. Res.1996, 296, 279-284

(2) Calarese, D. A.; Lee, H. K.; Huang, C. Y.; Best, M. D.; Astronomo, R. D.; Stanfield, R. L.; Katinger, H.; Burton, D.

R.; Wong, C. H.; Wilson, I. A., Proc. Natl. Acad. Sci. USA 2005, 102 (38), 13372-13377.

(3) Chouhan, G.; James, K. Org. Lett. 2011, 13, 2754-2757. 